\title{
CLT for linear spectral statistics of large dimensional sample covariance matrices with dependent data
}

\author{
Shurong Zheng, Zhidong Bai, Jianfeng Yao and Hongtu Zhu
}

July 13, 2016

\begin{abstract}
This paper investigates the central limit theorem for linear spectral statistics of high dimensional sample covariance matrices of the form $\mathbf{B}_{n}=n^{-1} \sum_{j=1}^{n} \mathbf{Q} \mathbf{x}_{j} \mathbf{x}_{j}^{*} \mathbf{Q}^{*}$ where $\mathbf{Q}$ is a nonrandom matrix of dimension $p \times k$, and $\left\{\mathbf{x}_{j}\right\}$ is a sequence of independent $k$-dimensional random vector with independent entries, under the assumption that $p / n \rightarrow y>0$. A key novelty here is that the dimension $k \geq p$ can be arbitrary, possibly infinity. This new model of sample covariance matrices $\mathbf{B}_{n}$ covers most of the known models as its special cases. For example, standard sample covariance matrices are obtained with $k=p$ and $\mathbf{Q}=\mathbf{T}_{n}^{1 / 2}$ for some positive definite Hermitian matrix $\mathbf{T}_{n}$. Also with $k=\infty$ our model covers the case of repeated linear processes considered in recent high-dimensional time series literature. The CLT found in this paper substantially generalizes the seminal CLT in Bai and Silverstein (2004). Applications of this new CLT are proposed for testing the structure of a high-dimensional covariance matrix. The derived tests are then used to analyse a large fMRI data set regarding its temporary correlation structure.
\end{abstract}

AMS 2000 subject Classifications: Primary 62H15; secondary 60F05,15B52.

Key word: Large sample covariance matrices, linear spectral statistics, central limit theorem, high-dimensional time series, high-dimensional dependent data.

Shurong Zheng and Zhidong Bai are professors in School of Mathematics \& Statistics and KLAS, Northeast Normal University, China; Jianfeng Yao is professor with the Department of Statistics and Actuarial Science, The University of Hong Kong, Hong Kong; Hongtu Zhu is professor at the University of North Caroline at Chapel Hill, USA. 


\section{Introduction}

Sample covariance matrices are of central importance in multivariate and high-dimensional data analysis. They have prominent applications in various big-data fields such as wireless communication networks, social networks and signal processing, see for instance the recent survey papers Couillet and Merouane (2013), Johnstone (2007) and Paul and Aue (2014). Many statistical tools in these area depend on the so-called linear spectral statistics (LSS) of a sample covariance matrix, say $\mathbf{B}_{n}$ of size $p \times p$ with eigenvalues $\left\{\lambda_{i}\right\}_{i=1}^{p}$, which have the form $p^{-1} \sum_{i=1}^{p} f\left(\lambda_{i}\right)$ where $f$ is a given function. Two main questions arise for such LSS, namely (i) determining the point limit of a limiting spectral distribution (LSD), say $G$, such that LSS converges to $G(f)=\int f(x) d G(x)$ (in an appropriate sense and for a wide family of functions $f$ ); and (ii) characterizing the fluctuations $p^{-1} \sum_{i=1}^{p} f\left(\lambda_{i}\right)-G(f)$ in terms of an appropriate central limit theorem (CLT). Both questions have a long history and continue to receive considerable attention in recent years. As for LSDs, the question has been extensively studied in the literature starting from the classical work of Marčenko and Pastur (1967), and contitued in Silverstein (1995) and Wachter (1980), and found many recent developments (Bai and Zhou, 2008; Yao, 2012; Banna and Merlevède, 2015, among others.)

As for CLTs for linear spectral statistics, a CLT for $\left(\operatorname{tr}\left(\mathbf{B}_{n}\right), \cdots, \operatorname{tr}\left(\mathbf{B}_{n}^{\ell}\right)\right)$ is established in Jonsson (1982) for a sequence of Wishart matrices $\left\{\mathbf{B}_{n}\right\}$ with Gaussian variables, where $\ell$ is a fixed number, and the dimension $p$ of the matrices grows proportionally to the sample size $n$. By employing a general method based on Stieltjes transform, Bai and Silverstein (2004) provides a CLT for LSS of large dimensional sample covariance matrices from a general population, that is, not necessarily Gaussian along with an arbitrary population covariance matrix. A distinguished feature of this CLT is that its centering term and limiting means and covariance functions are all fully characterized. This celebrated paper has been subsequently improved in several follow-up papers including Pan and Zhou (2008), Pan (2012), Najim and Yao (2013) and Zheng, Bai and Yao (2015). These CLTs have found successful applications in high-dimensional statistics by solving notably important problems in parameter estimation or hypothesis testing with high-dimensional data. Examples include Bai et al. (2009), Wang and Yao (2013) and Liu et al. (2014) for hypothesis testing on covariance matrix; Bai et al. (2013) for testing on regression coefficients, and Jiang, Bai and Zheng (2013) for testing indepedence between two large sets of variables, among others.

The aim of this paper is to establish a new CLT for a much extended class of sample covariance matrices. This new development represents a novel and major extension of the 
CLT of Bai and Silverstein (2004) and its recent follow-up version. Specifically, in this paper, we consider samples $\left\{\mathbf{y}_{j}\right\}_{1 \leq j \leq n}$ of the form $\mathbf{y}_{j}=\mathbf{Q} \mathbf{x}_{j}$ where

(M1) $\left\{\mathbf{x}_{j}=\left(x_{1 j}, \ldots, x_{k j}\right)^{T}, 1 \leq j \leq n\right\}$ is a sequence of independent $k$-dimensional random vectors with independent standardized components $x_{i j}$, i.e. $E x_{i j}=0$ and $E\left|x_{i j}\right|^{2}=1$, and the dimension $k \geq p$, possibly $k=\infty$ (for example, each $\mathbf{x}_{j}$ is a white noise made with an infinite sequence of standardized errors);

(M2) $\mathbf{Q}$ is a $p \times k$ matrix with arbitrary entries.

The sample covariance matrix in our setting is then given by

$$
\mathbf{B}_{n}=n^{-1} \sum_{j=1}^{n} \mathbf{y}_{j} \mathbf{y}_{j}^{*}=n^{-1} \mathbf{Q} \mathbf{X}_{n} \mathbf{X}_{n}^{*} \mathbf{Q}^{*}
$$

where $\mathbf{X}_{n}=\left(\mathbf{x}_{1}, \ldots, \mathbf{x}_{n}\right)$ is an $k \times n$ matrix (with independent entries) and $*$ stands for the transpose and complex conjugate of matrices or vectors. In contrast, classical sample covariance matrices as in Bai and Silverstein (2004) consider samples of the form $\mathbf{y}_{j}=\mathbf{C}_{n}^{1 / 2} \mathbf{x}_{j}$, where

(O1) $\left\{\mathbf{x}_{j}=\left(x_{1 j}, \ldots, x_{p j}\right)^{T}, 1 \leq j \leq n\right\}$ is a sequence of iid $p$-dimensional random vectors with independent standardized components $x_{i j}$, i.e. $E x_{i j}=0$ and $E\left|x_{i j}\right|^{2}=1$.

(O2) $\mathbf{C}_{n}^{1 / 2}$ is a $p \times p$ positive definite Hermitian matrix.

The sample covariance matrix of interest thus takes form

$$
\mathbf{S}_{n}=n^{-1} \sum_{i=1}^{n} \mathbf{y}_{j} \mathbf{y}_{j}^{*}=n^{-1} \mathbf{C}_{n}^{1 / 2} \mathbf{X}_{n} \mathbf{X}_{n}^{*} \mathbf{C}_{n}^{1 / 2}
$$

where $\mathbf{X}_{n}=\left(\mathbf{x}_{1}, \ldots, \mathbf{x}_{n}\right)$ is a $p \times n$ data matrix (with independent entries).

The main innovation in the model (1.1) is that the mixing matrix $\mathbf{Q}$ can have an infinite number of columns, and this will allow to cover dependent samples. For example, the repeated linear process $\left\{y_{i j}=\sum_{t=-\infty}^{\infty} b_{t} x_{i-t, j}, i=1, \ldots, p, j=1, \ldots, n\right\}$ is a special case of (1.1) with

$$
\mathbf{y}_{j}=\mathbf{Q x}_{j}=\underbrace{\left(\begin{array}{cccccccc}
\cdots & b_{1-p} & b_{2-p} & \cdots & b_{0} & b_{1} & b_{2} & \cdots \\
\vdots & \vdots & \vdots & \vdots & \vdots & \vdots & \vdots & \\
\cdots & b_{-1} & b_{0} & \ldots & b_{p-2} & b_{p-1} & b_{p} & \cdots \\
\cdots & b_{0} & b_{1} & \ldots & b_{p-1} & b_{p} & b_{p+1} & \cdots
\end{array}\right)}_{\mathbf{Q} \text { is } p \times \infty \text { dimensional }} .
$$


Such data structure often arises in panel surveys or longitudinal studies, in which respondents or subjects are interviewed/observed during a certain time period (see Wong and Miller (1990); Wong. Miller and Shrestha (2001)). Moreover, if indeed $k=p$, then (1.2) of Bai and Silverstein (2004) is a special case of our novel extension. In this case, although the entries of $p \times p$ matrix $\mathbf{Q}$ are arbitrary and $\mathbf{Q}$ is not required to be Hermitian or positive definite, our setup is in fact strictly equivalent to Bai and Silverstein (2004) due to the polar decomposition $\mathbf{Q}=\mathbf{U}_{n} \mathbf{T}_{n}^{1 / 2}$, in which $\mathbf{U}_{n}$ is unitary and $\mathbf{T}_{n}^{1 / 2}$ is Hermitian and nonnegative definite. Consequently, $\mathbf{B}_{n}=\mathbf{U}_{n} \mathbf{S}_{n} \mathbf{U}_{n}^{*}$ where $\mathbf{S}_{n}$ is equivalent to the sample covariance matrix in (1.2) has been considered in Bai and Silverstein (2004). Therefore, $\mathbf{B}_{n}$ and $\mathbf{S}_{n}$ have exactly the same spectrum of eigenvalues and their LSS are identical. However, when $k$ tends to infinity with a higher order than $p$, the new model in (1.1) represents a non-trivial extension of the framework of Bai and Silverstein (2004).

We will derive the LSD of the sample covariance matrix $\mathbf{B}_{n}$ in (1.1) and the corresponding CLT for its linear spectral statistics, thus extending both the results of Bai and Silverstein (2004) and Silverstein (1995) into the new framework. As it will be seen below, establishing these extensions requires several novel ideas and major techniques in order to tackle with a mixing matrix $\mathbf{Q}$ that can be infinite and with arbitrary entries.

The rest of the paper is organized as follows. In Section 2, we derive the LSD of the sample covariance matrix $\mathbf{B}_{n}$ under suitable conditions. In Section 3, we derive the CLT for linear spectral statistics of $\mathbf{B}_{n}$ under some reinforced conditions. In Section 4 , we present two applications of these theoretical results. Section 5 collects the proofs for the main theorems of the paper. Finally, some technical lemmas and auxiliary results used in the proofs of Section 5 are postponed to the appendices.

\section{LSD of the sample covariance matrix $\mathbf{B}_{n}$}

This section aims at the derivation of the LSD for the sample covariance matrix $\mathbf{B}_{n}$ in (1.1) from sample $\mathbf{y}_{j}$ 's of dependent data. Recall that the empirical spectral distribution (ESD) of a $p \times p$ square matrix $\mathbf{A}$ is the probability measure $F^{\mathbf{A}}=p^{-1} \sum_{i=1}^{p} \delta_{\lambda_{i}}$, where the $\lambda_{i}$ 's are eigenvalues of $\mathbf{A}$ and $\delta_{a}$ denotes the Dirac mass at point $a$. For any probability measure $F$ on the real line, its Stieltjes transform is defined by

$$
m(z)=\int \frac{1}{t-z} d F(x), \quad z \in \mathbb{C}^{+}=\{z \in \mathbb{C}: \Im(z)>0\},
$$

where $\mathbb{C}$ denotes the complex plane and $\Im$ the imaginary part.

The assumptions needed for the derivation of the LSD of $\mathbf{B}_{n}$ are as follows. 
Assumption (a) Samples are $\left\{\mathbf{y}_{j}=\mathbf{Q} \mathbf{x}_{j}, j=1, \ldots, n\right\}$, where $\mathbf{Q}$ is $p \times k, \mathbf{x}_{j}$ is $k \times 1$, $\mathbf{x}_{j}=\left(x_{1 j}, \ldots, x_{k j}\right)^{T}$, and the dimension $k$ is arbitrary (possibly infinite). Moreover, $\left\{x_{i j}, i=1, \ldots, k, j=1, \ldots, n\right\}$ is a $k \times n$ array of independent random variables, not necessarily identically distributed, with common moments

$$
E x_{i j}=0, \quad E\left|x_{i j}^{2}\right|=1
$$

and satisfying the following Lindeberg-type condition: for each $\eta>0$,

$$
\frac{1}{p n \eta^{2}} \sum_{i=1}^{k} \sum_{j=1}^{n}\left\|\mathbf{q}_{i}\right\|^{2} \mathrm{E}\left|x_{i j}^{2}\right| I\left(\left|x_{i j}\right|>\eta \sqrt{n} /\left\|\mathbf{q}_{i}\right\|\right) \rightarrow 0
$$

where $\left\|\mathbf{q}_{i}\right\|$ is the Euclidean norm ( $k$ is finite) or the $\ell^{2}$ norm $(k=\infty)$ of the $i$-th column vector $\mathbf{q}_{i}$ of $\mathbf{Q}$.

Assumption (b) With probability one, the ESD $H_{n}$ of the population covariance matrix $\mathbf{T}_{n}=\mathrm{QQ}^{*}$ converges weakly to a probability distribution $H$. Also the sequence $\left(\mathbf{T}_{n}\right)_{n}$ is bounded in spectral norm.

Assumption (c) Both $p$ and $n$ tend to infinity such that $y_{n}=p / n \rightarrow y>0$ as $n \rightarrow \infty$.

The Lindeberg-type condition in Assumption (a) is a classical moment condition of second order. It is automatically satisfied if the entries $\left\{x_{i j}\right\}$ are identically distributed. This condition is here used to tackle with possibly non identically distributed entries and ensures a suitable truncation of the variables. Assumption (b) is also a standard condition on the convergence of the population spectral distribution and requires the weak convergence of the ESD of the population covairance matrix. Assumption (c) defines the asymptotic regime following the seminal work of Marčenko and Pastur (1967).

As the first main result of the paper, we derive the LSD of $\mathbf{B}_{n}$.

Theorem 2.1. Under Assumptions (a), (b) and (c), almost surely the ESD $F^{\mathbf{B}_{n}}$ of $\mathbf{B}_{n}$ weakly converges to a non-random LSD $F^{y, H}$. Moreover, the LSD $F^{y, H}$ is determined through its Stieltjes transform $m(z)$, which is the unique solution to the following MarčenkoPastur equation

$$
m(z)=\int \frac{1}{t[1-y-y z m(z)]-z} d H(t),
$$

on the set $\left\{m(z) \in \mathbb{C}:-(1-y) / z+y m(z) \in \mathbb{C}^{+}\right\}$.

Theorem 2.1 has several important implications. The LSD $F^{y, H}$ is exactly the generalized Marčenko-Pastur distribution with parameters $(y, H)$ as described in Silverstein 
(1995). The key novelty of Theorem 2.1 consists of an extension of this LSD into a much more general setting on matrix entries as defined in Assumptions (a)-(b). Therefore, Theorem 2.1 includes many existing results related to LSDs of sample covariance matrices from dependent data as special cases, e.g. Bai and Zhou (2008); Jin et al. (2014); Jin, Wang and Miao (2009); Wang. Jin and Miao (2011); Yao (2012). To the best of our knowledge, although the model considered in Bai and Zhou (2008) is more general than that in this paper, it relies on a strong quadratic form condition, which can be hardly verified in many applications.

Further developments on this LSD rely on an equivalent representation of the MarčenkoPastur law. Namely, define the companion $L S D$ of $\mathbf{B}_{n}$ as

$$
\underline{F}^{y, H}=(1-y) \delta_{0}+y F^{y, H}
$$

It is readily checked that $\underline{F}^{y, H}$ is the LSD of the companion sample covariance matrix $\underline{\mathbf{B}}_{n}=n^{-1} \mathbf{X}_{n}^{*} \mathbf{Q}^{*} \mathbf{Q} \mathbf{X}_{n}$ (which is $n \times n$ ), and its Stieltjes transform $\underline{m}(z)$ satisfies the socalled Silverstein equation

$$
z=-\frac{1}{\underline{m}(z)}+y \int \frac{t}{1+t \underline{m}(z)} d H(t), \quad \underline{m}(z)=-\frac{1-y}{z}+y m(z) .
$$

The advantage of this equation is that it indeed defines the inverse function of the Stieltjes transform $\underline{m}(z)$. This equation is the key to numerical evaluation of the Stieltjes transform, or the underlying density function of the LSD. Moreover, many analytical properties on LSD can be inferred from this equation (see Silverstein and Choi (1995)).

We consider an example to elaborate more on Theorem 2.1 ,

Example 2.1. Assume that for each $1 \leq j \leq n,\left\{y_{i j}\right\}_{i}$ is an $A R M A(1,1)$ process of the form

$$
y_{i j}=\phi y_{i-1, j}+\theta \varepsilon_{i-1, j}+\varepsilon_{i j}
$$

where $\left\{\varepsilon_{i j}\right\}$ is an array of independently and identically distributed random variables with mean zero and variance 1. The condition $|\phi| \vee|\theta|<1$ is assumed for causality and invertibility of this $\operatorname{ARMA}(1,1)$ process. From (2.1) or (2.2), it follows that the Stieltjes transform $m(z)$ of the $L S D F^{y, H}$ satisfies the following equation

$$
z=-\frac{1}{m(z)}+\frac{\theta}{y \theta m(z)-\phi}-\frac{(\phi+\theta)(1+\phi \theta)}{(y \theta m(z)-\phi)^{2}} \frac{\epsilon(\alpha)}{\sqrt{\alpha^{2}-4}}
$$

with

$$
\alpha=\frac{y m(z)\left(1+\theta^{2}\right)+1+\phi^{2}}{y \theta m(z)-\phi} \text { and } \epsilon(\alpha)=\operatorname{sgn}(\Im(\alpha)) \text {. }
$$


In the case of an $A R(1)$ process $(\theta=0)$, we have

$$
z=-\frac{1}{m(z)}+\frac{1}{\sqrt{\left[y m(z)+1+\phi^{2}\right]^{2}-4 \phi^{2}}} .
$$

Similarly, in the case of a $M A(1)$ processs $(\phi=0)$, we have

$$
z=-\frac{1}{m(z)}+\frac{1}{y m(z)}+\frac{1}{y^{2} m^{2}(z)} \frac{1}{\sqrt{\left\{[y m(z)]^{-1}+1+\theta^{2}\right\}^{2}-4 \theta^{2}}}
$$

(see Jin, Wang and Miao (2009), Wang, Jin and Miao (2011) and Yao (2012)).

Remark 1. In general, we cannot find a closed-form formula for the Stieltjes transform $m(z)$ (or $\underline{m}(z))$. One has to rely on numerical evaluation using the Silverstein equation (2.2). Notice that the values of $m(z)$ at points $z=x+\mathbf{i} \varepsilon$ with small positive $\varepsilon$ provides an approximation to the value of the density of the LSD at $x$ by the inversion formula. For details on these numerical algorithms, we refer to Dobriban (2015). By (2.2) and Silverstein and Choi (1995), we obtain $\underline{m}(z)$ and the limiting density $f^{y, H}$ of $F^{y, H}$, which satisfies $f^{y, H}(x)=(y \pi)^{-1} \lim _{z \rightarrow x+0 \mathbf{i}} \Im(\underline{m}(z))$.

Remark 2. If $\mathbf{T}_{n}=\mathbf{Q Q}^{*}$ is the identity matrix $\mathbf{I}_{p}$, then $H=\delta_{1}$ and we have by (2.2),

$$
z=-\frac{1}{\underline{m}(z)}+\frac{y}{1+\underline{m}(z)} .
$$

That is,

$$
\underline{m}(z)=\frac{-(z+1-y)+\sqrt{(z-1-y)^{2}-4 y}}{2 z}
$$

and the LSD $F^{y, H}$ of $\mathbf{B}_{n}$ is the Marčenko-Pastur law with the density function

$$
f^{y, H}=(2 \pi y x)^{-1} \sqrt{\left[(1+\sqrt{y})^{2}-x\right]\left[x-(1-\sqrt{y})^{2}\right]}
$$

and has a point mass $1-1 / y$ at the origin if $y>1$.

\section{CLT for linear spectral statistics of the sample co- variance matrix $\mathbf{B}_{n}$}

In this section, we establish the corresponding CLT for LSS of the sample covariance matrix $\mathbf{B}_{n}$ in (1.1) under approriate conditions. This CLT constitutes the second main contribution of this paper. We first introduce the assumptions needed for this CLT. 
Assumption (d) The variables $\left\{x_{i j}, i=1, \ldots, k, j=1, \ldots, n\right\}$ are independent, with common moments

$$
E x_{i j}=0, \quad E\left|x_{i j}^{2}\right|=1, \quad \beta_{x}=E\left|x_{i j}^{4}\right|-\left|E x_{i j}^{2}\right|^{2}-2, \quad \text { and } \quad \alpha_{x}=\left|E x_{i j}^{2}\right|^{2},
$$

and satisfying the following Lindeberg-type condition: for each $\eta>0$

$$
\frac{1}{p n \eta^{6}} \sum_{i=1}^{k} \sum_{j=1}^{n}\left\|\mathbf{q}_{i}\right\|^{2} \mathrm{E}\left|x_{i j}^{4}\right| I\left(\left|x_{i j}\right|>\eta \sqrt{n /\left\|\mathbf{q}_{i}\right\|}\right) \rightarrow 0
$$

Assumption (e) Either $\alpha_{x}=0$, or the mixing matrix $\mathbf{Q}$ is real (with arbitrary $\alpha_{x}$ ).

Assumption (f) Either $\beta_{x}=0$, or the mixing matrix $\mathbf{Q}$ is such that diagonal $\mathbf{Q}^{*} \mathbf{Q}$ (with arbitrary $\left.\beta_{x}\right)$.

Remark 3. Assumption (d) is stronger than Assumption (a) in Section 2 since Assumption (d) assumes the Lindeberg-type condition on the fourth order moments (instead of the previous moments of second order). Assumption (e) means that if variables $x_{i j} s$ ' are complex-valued, $E\left(x_{i j}^{2}\right)=0$ should hold which is the Gaussian-like second moment in Bai and Silverstein (2004). However, if the other real $\mathbf{Q}$ condition is imposed, then the Gaussian-like second moment can be removed $\left(\alpha_{x} \neq 0\right)$. If $\alpha_{x} \neq 0$ and $\mathbf{Q}$ is not real, then the CLT for LSS of $\mathbf{B}_{n}$ may not hold. Such counterexample for the case of $k=p$ can be found in Zheng, Bai and Yao (2015). As for Assumption (f), if the population is Gaussian, then $\beta_{x}=0$ is the Gaussian-like fourth moment. If we impose the condition that the matrix $\mathbf{Q}^{*} \mathbf{Q}$ is real and diagonal, then the Gaussian-like fourth moment condition can be removed. Again if $\beta_{x} \neq 0$ and $\mathbf{Q}^{*} \mathbf{Q}$ is not diagonal, the CLT may not hold. Such counterexamples with $k=p$ can be found in Zheng. Bai and Yao (2015).

Theorem 3.1. Under Assumptions (b)-(f), let $f_{1}, \ldots, f_{L}$ be L functions analytic on a complex domain containing

$$
\left[I_{(0<y<1)}(1-\sqrt{y})^{2} \liminf _{n} \lambda_{\min }^{\mathbf{T}_{n}}, \quad(1+\sqrt{y})^{2} \limsup _{n} \lambda_{\max }^{\mathbf{T}_{n}}\right]
$$

with $\mathbf{T}_{n}=\mathbf{Q Q}^{*}$, and $\lambda_{\min }^{\mathbf{T}_{n}}$ and $\lambda_{\max }^{\mathbf{T}_{n}}$ denoting its smallest and the largest eigenvalue, respectively. Consider the random vector $\left(X_{p}\left(f_{1}\right), \ldots, X_{p}\left(f_{L}\right)\right)$ with

$$
X_{p}\left(f_{\ell}\right)=\sum_{j=1}^{p} f_{\ell}\left(\lambda_{j}\right)-p F^{y_{n}, H_{n}}\left(f_{\ell}\right), \quad \ell=1, \ldots, L
$$


in which $F^{y_{n}, H_{n}}\left(f_{\ell}\right)=\int f_{\ell}(x) f^{y_{n}, H_{n}}(x) d x$ and $\left\{\lambda_{j}\right\}_{j=1}^{p}$ are the sample eigenvalues of $\mathbf{B}_{n}$. Then, the random vector $\left(X_{p}\left(f_{1}\right), \ldots, X_{p}\left(f_{L}\right)\right)$ converges to a L-dimensional Gaussian random vector $\left(X_{f_{1}}, \ldots, X_{f_{L}}\right)$ with mean function

$$
\begin{aligned}
\mathrm{E}_{f_{\ell}}= & -\frac{1}{2 \pi \mathbf{i}} \oint_{\mathcal{C}} f_{\ell}(z) \frac{\alpha_{x} y \int \frac{\underline{m}^{3}(z) t^{2}}{(1+\underline{m}(z))^{3}} d H(t)}{\left(1-y \int \frac{\underline{m}^{2}(z) t^{2}}{(1+t \underline{t m}(z))^{2}} d H(t)\right)\left(1-\alpha_{x} y \int \frac{\underline{m}^{2}(z) t^{2}}{(1+t \underline{m}(z))^{2}} d H(t)\right)} d z \\
& -\frac{\beta_{x}}{2 \pi \mathbf{i}} \oint_{\mathcal{C}} f_{\ell}(z) \frac{y \int \frac{\underline{m}^{3}(z) t^{2}}{(\underline{m}(z) t+1)^{3}} d H(t)}{1-y \int \frac{\underline{m}^{2}(z) t^{2} d H(t)}{(1+t \underline{m}(z))^{2}}} d z,
\end{aligned}
$$

and variance-covariance function

$$
\begin{aligned}
& \operatorname{Cov}\left(X_{f_{\ell^{\prime}}}, X_{f_{\ell}}\right) \\
= & -\frac{1}{4 \pi^{2}} \oint_{\mathcal{C}_{1}} \oint_{\mathcal{C}_{2}} \frac{f_{\ell^{\prime}}\left(z_{1}\right) f_{\ell}\left(z_{2}\right)}{\left(\underline{m}\left(z_{1}\right)-\underline{m}\left(z_{2}\right)\right)^{2}} \frac{\partial \underline{m}\left(z_{1}\right)}{\partial z_{1}} \frac{\partial \underline{m}\left(z_{2}\right)}{\partial z_{2}} d z_{1} d z_{2} \\
& -\frac{y \beta_{x}}{4 \pi^{2}} \oint_{\mathcal{C}_{1}} \oint_{\mathcal{C}_{2}} f_{\ell^{\prime}}\left(z_{1}\right) f_{\ell}\left(z_{2}\right)\left[\int \frac{t}{\left(\underline{m}\left(z_{1}\right) t+1\right)^{2}} \frac{t}{\left(\underline{m}\left(z_{2}\right) t+1\right)^{2}} d H(t)\right] \frac{\partial \underline{m}\left(z_{1}\right)}{\partial z_{1}} \frac{\partial \underline{m}\left(z_{2}\right)}{\partial z_{2}} d z_{1} d z_{2} \\
& +\frac{1}{4 \pi^{2}} \oint_{\mathcal{C}_{1}} \oint_{\mathcal{C}_{2}} f_{\ell^{\prime}}\left(z_{1}\right) f_{\ell}\left(z_{2}\right)\left[\frac{\partial^{2}}{\partial z_{1} \partial z_{2}} \log \left(1-a\left(z_{1}, z_{2}\right)\right)\right] d z_{1} d z_{2},
\end{aligned}
$$

where $\mathcal{C}, \mathcal{C}_{1}$ and $\mathcal{C}_{2}$ are closed contours in the complex plane enclosing the support of the $L S D F^{y, H}$, and $\mathcal{C}_{1}$ and $\mathcal{C}_{2}$ are non-overlapping. Moreover, $a\left(z_{1}, z_{2}\right)$ is given by

$$
a\left(z_{1}, z_{2}\right)=\alpha_{x}\left(1+\frac{\underline{m}\left(z_{1}\right) \underline{m}\left(z_{2}\right)\left(z_{1}-z_{2}\right)}{\underline{m}\left(z_{2}\right)-\underline{m}\left(z_{1}\right)}\right) .
$$

Remark 4. Theorem 3.1 gives the explicit form for the mean and covariance functions of $X_{f_{\ell}}$ expressed by contour integrals. For some specific function $f_{\ell}$ and $\mathbf{Q}$, the mean and covariance functions of $X_{f_{\ell}}$ can be explicitly obtained. Even if the mean and covariance functions of $X_{f_{\ell}}$ have no explicit forms, numerical methods can be used since the integrals are at most two-dimensional. If the population is Gaussian, then $\beta_{x}=0$ and the corresponding terms in both the limiting mean and covariance functions vanish. If the population is real, then we have $\alpha_{x}=1$ and

$$
\frac{\partial^{2} \log \left(1-a\left(z_{1}, z_{2}\right)\right)}{\partial z_{1} \partial z_{2}}=\frac{-1}{\left(\underline{m}\left(z_{1}\right)-\underline{m}\left(z_{2}\right)\right)^{2}} \frac{\partial \underline{m}\left(z_{1}\right)}{\partial z_{1}} \frac{\partial \underline{m}\left(z_{2}\right)}{\partial z_{2}} .
$$

Notice that the limiting mean and covariance functions of $\left\{X_{f_{\ell}}\right\}$ depend on the LSD $H$ of $\mathrm{QQ}^{*}$ and $y$ only. In many applications, the ESD $H_{n}$ of $\mathbf{Q Q}^{*}$ and the ratio $y_{n}=p / n$ can 
be used to replace the LSD $H$ and the limit $y$. But it is worthwhile to mention that when $\mathbf{B}_{n}$ is the centered sample covariance matrix $\mathbf{B}_{n}=(n-1)^{-1} \sum_{i=1}^{n}\left(\mathbf{y}_{i}-\overline{\mathbf{y}}\right)\left(\mathbf{y}_{i}-\overline{\mathbf{y}}\right)^{T}$ with $\overline{\mathbf{y}}=n^{-1} \sum_{i=1}^{n} \mathbf{y}_{i}, y_{n}$ in (3.3) should be replaced by $y_{n-1}$. For example, $\mathbf{y}_{i} \sim N\left(\mathbf{0}_{p}, \mathbf{I}_{p}\right)$, then the Wishart matrices $n^{-1} \sum_{i=1}^{n} \mathbf{y}_{i} \mathbf{y}_{i}^{T}$ and $(n-1)^{-1} \sum_{i=1}^{n}\left(\mathbf{y}_{i}-\overline{\mathbf{y}}\right)\left(\mathbf{y}_{i}-\overline{\mathbf{y}}\right)^{T}$ have degrees of freedoms $n$ and $n-1$, respectively.

For practical use, by Theorem 3.1 and Remark 2, we will give three corollaries that are useful for computing the variance and covariance of the limiting distribution.

Corollary 3.1. Under the conditions of Theorem 3.1 and if the population is real, then the random vector $\left(X_{p}\left(f_{1}\right), \ldots, X_{p}\left(f_{L}\right)\right)$ converges to an L-dimensional Gaussian random vector $\left(X_{f_{1}}, \ldots, X_{f_{L}}\right)$ with mean function

$\mathrm{E} X_{f_{\ell}}=-\frac{1}{2 \pi \mathbf{i}} \oint_{\mathcal{C}} f_{\ell}(z) \frac{y \int \frac{\underline{m}^{3}(z) t^{2}}{(1+t \underline{\underline{m}}(z))^{3}} d H(t)}{\left(1-y \int \frac{\underline{m}^{2}(z) t^{2}}{(1+\underline{\underline{m}}(z))^{2}} d H(t)\right)^{2}} d z-\frac{\beta_{x}}{2 \pi \mathbf{i}} \oint_{\mathcal{C}} f_{\ell}(z) \frac{y \int \frac{\underline{m}^{3}(z) t^{2}}{(\underline{m}(z) t+1)^{3}} d H(t)}{1-y \int \frac{\underline{m}^{2}(z) t^{2} d H(t)}{(1+t \underline{m}(z))^{2}}} d z$,

and variance-covariance function

$$
\begin{aligned}
& \operatorname{Cov}\left(X_{f_{\ell^{\prime}}}, X_{f_{\ell}}\right) \\
= & -\frac{1}{2 \pi^{2}} \oint_{\mathcal{C}_{1}} \oint_{\mathcal{C}_{2}} \frac{f_{\ell^{\prime}}\left(z_{1}\right) f_{\ell}\left(z_{2}\right)}{\left(\underline{m}\left(z_{1}\right)-\underline{m}\left(z_{2}\right)\right)^{2}} \frac{\partial \underline{m}\left(z_{1}\right)}{\partial z_{1}} \frac{\partial \underline{m}\left(z_{2}\right)}{\partial z_{2}} d z_{1} d z_{2} \\
& -\frac{y \beta_{x}}{4 \pi^{2}} \oint_{\mathcal{C}_{1}} \oint_{\mathcal{C}_{2}} f_{\ell^{\prime}}\left(z_{1}\right) f_{\ell}\left(z_{2}\right)\left[\int \frac{t}{\left(\underline{m}\left(z_{1}\right) t+1\right)^{2}} \frac{t}{\left(\underline{m}\left(z_{2}\right) t+1\right)^{2}} d H(t)\right] \frac{\partial \underline{m}\left(z_{1}\right)}{\partial z_{1}} \frac{\partial \underline{m}\left(z_{2}\right)}{\partial z_{2}} d z_{1} d z_{2},
\end{aligned}
$$

where $\mathcal{C}, \mathcal{C}_{1}$ and $\mathcal{C}_{2}$ are closed contours in the complex plane enclosing the support of the LSD $F^{y, H}$, and $\mathcal{C}_{1}$ and $\mathcal{C}_{2}$ are non-overlapping.

Corollary 3.2. Under the assumptions of Theorem 3.1, if the population is real and $H_{n}=$ $\delta_{1}$, then we have

$$
\begin{aligned}
F^{y, H_{n}}\left(f_{\ell}\right)= & \int_{(1-\sqrt{y})^{2}}^{(1+\sqrt{y})^{2}} \frac{f_{\ell}(x)}{2 \pi y x} \sqrt{\left[(1+\sqrt{y})^{2}-x\right]\left[x-(1-\sqrt{y})^{2}\right]} d x+(1-1 / y) \operatorname{sgn}(y>1) f_{\ell}(0), \\
\mathrm{E} X_{f_{\ell}}= & \frac{f_{\ell}\left((1-\sqrt{y})^{2}\right)+f_{\ell}\left((1+\sqrt{y})^{2}\right)}{4}-\frac{1}{2 \pi} \int_{(1-\sqrt{y})^{2}}^{(1+\sqrt{y})^{2}} \frac{f_{\ell}(x)}{\sqrt{4 y-(x-1-y)^{2}}} d x \\
& -\frac{\beta_{x}}{2 \pi \mathbf{i}} \oint_{\mathcal{C}} f_{\ell}(z) \frac{y \underline{m}^{3}(z)(\underline{m}(z)+1)^{-3}}{1-y \underline{m}^{2}(z)(1+\underline{m}(z))^{-2}} d z,
\end{aligned}
$$


and

$$
\begin{aligned}
\operatorname{Cov}\left(X_{f_{\ell^{\prime}}}, X_{f_{\ell}}\right)= & -\frac{1}{2 \pi^{2}} \oint_{\mathcal{C}_{1}} \oint_{\mathcal{C}_{2}} \frac{f_{\ell^{\prime}}\left(z_{1}\right) f_{\ell}\left(z_{2}\right)}{\left(\underline{m}\left(z_{1}\right)-\underline{m}\left(z_{2}\right)\right)^{2}} \frac{\partial \underline{m}\left(z_{1}\right)}{\partial z_{1}} \frac{\partial \underline{m}\left(z_{2}\right)}{\partial z_{2}} d z_{1} d z_{2} \\
& -\frac{y \beta_{x}}{4 \pi^{2}} \oint_{\mathcal{C}} \frac{f_{\ell^{\prime}}\left(z_{1}\right)}{\left(\underline{m}\left(z_{1}\right)+1\right)^{2}} \frac{\partial \underline{m}\left(z_{1}\right)}{\partial z_{1}} d z_{1} \oint_{\mathcal{C}} \frac{f_{\ell}\left(z_{2}\right)}{\left(\underline{m}\left(z_{2}\right)+1\right)^{2}} \frac{\partial \underline{m}\left(z_{2}\right)}{\partial z_{2}} d z_{2},
\end{aligned}
$$

where $\mathcal{C}, \mathcal{C}_{1}$ and $\mathcal{C}_{2}$ are closed contours in the complex plane enclosing the support [(1$\left.\sqrt{y})^{2},(1+\sqrt{y})^{2}\right]$ of the $L S D F^{y, H}, \mathcal{C}_{1}$ and $\mathcal{C}_{2}$ are non-overlapping, and $z=-\underline{m}^{-1}(z)+$ $y(1+\underline{m}(z))^{-1}$.

If the real samples $\left\{\mathbf{y}_{i}, i=1, \ldots, n\right\}$ are transformed as $\left\{\left(\mathbf{Q Q}^{*}\right)^{-1 / 2} \mathbf{y}_{i}, i=1, \ldots, n\right\}$, then the population covariance matrix of $\left(\mathbf{Q Q}^{*}\right)^{-1 / 2} \mathbf{y}_{i}$ is the identity matrix $\mathbf{I}_{p}$. Then $H_{n}=\delta_{1}$. By Corollary 3.2 and Remark 4, we give below a corollary useful for highdimensional statistical inference.

Corollary 3.3. Under the assumptions of Theorem 3.1, if the population is such that $H_{n}=\delta_{1}$, then we have

$$
\left\{\operatorname{tr}\left[\left(\mathbf{Q Q}^{*}\right)^{-1} \mathbf{B}_{n}\right]-p, \ldots, \operatorname{tr}\left[\left(\mathbf{Q Q}^{*}\right)^{-1} \mathbf{B}_{n}\right]^{L}-p F^{(L)}\left(y_{n}\right)\right\}^{T} \rightarrow N(\boldsymbol{\mu}, \boldsymbol{\Sigma})
$$

where $\mathbf{B}_{n}=n^{-1} \sum_{i=1}^{n}\left(\mathbf{y}_{i}-\mathrm{E} \mathbf{y}_{i}\right)\left(\mathbf{y}_{i}-\mathrm{E}_{i}\right)^{T}, \boldsymbol{\mu}=\left(\mu_{1}, \ldots, \mu_{L}\right)^{T}$ and $\boldsymbol{\Sigma}=\left(\sigma_{\ell \ell^{\prime}}\right)_{\ell, \ell^{\prime}=1}^{L}$. Moreover, if $\mathbf{B}_{n}=(n-1)^{-1} \sum_{i=1}^{n}\left(\mathbf{y}_{i}-\overline{\mathbf{y}}\right)\left(\mathbf{y}_{i}-\overline{\mathbf{y}}\right)^{T}$ with the sample mean $\overline{\mathbf{y}}=n^{-1} \sum_{i=1}^{n} \mathbf{y}_{i}$, then we have

$$
\left\{\operatorname{tr}\left[\left(\mathbf{Q Q}^{*}\right)^{-1} \mathbf{B}_{n}\right]-p, \ldots, \operatorname{tr}\left[\left(\mathbf{Q Q}^{*}\right)^{-1} \mathbf{B}_{n}\right]^{L}-p F^{(L)}\left(y_{n-1}\right)\right\}^{T} \rightarrow N(\boldsymbol{\mu}, \boldsymbol{\Sigma}) .
$$

Here,

$$
\begin{aligned}
F^{(\ell)}(y)= & \int_{(1-\sqrt{y})^{2}}^{(1+\sqrt{y})^{2}} \frac{x^{\ell-1}}{2 \pi y} \sqrt{\left[(1+\sqrt{y})^{2}-x\right]\left[x-(1-\sqrt{y})^{2}\right]} d x, \quad \ell=1, \ldots, L, \\
\mu_{\ell}= & \frac{(1-\sqrt{y})^{2 \ell}+(1+\sqrt{y})^{2 \ell}}{4}-\frac{1}{2} \sum_{\ell_{1}=0}^{\ell}\left(\begin{array}{c}
\ell \\
\ell_{1}
\end{array}\right)^{2} y^{\ell} \\
& +\beta_{x} \operatorname{sgn}(\ell \geq 2) \sum_{\ell_{2}=2}^{\ell}\left(\begin{array}{c}
\ell \\
\ell_{2}-2
\end{array}\right)\left(\begin{array}{c}
\ell \\
\ell_{2}
\end{array}\right) y^{\ell+1-\ell_{2}}, \quad \ell=1, \ldots, L,
\end{aligned}
$$

and

$\sigma_{\ell \ell^{\prime}}=$ 


$$
\begin{aligned}
& 2 y^{\ell+\ell^{\prime}} \sum_{\ell_{1}=0}^{\ell-1} \sum_{\ell_{2}=0}^{\ell^{\prime}}\left(\begin{array}{l}
\ell \\
\ell_{1}
\end{array}\right)\left(\begin{array}{l}
\ell^{\prime} \\
\ell_{2}
\end{array}\right)\left(\frac{1-y}{y}\right)^{\ell_{1}+\ell_{2}} \sum_{\ell_{3}=0}^{\ell-\ell_{1}} \ell_{3}\left(\begin{array}{c}
2 \ell-1-\ell_{1}-\ell_{3} \\
\ell-1
\end{array}\right)\left(\begin{array}{c}
2 \ell^{\prime}-1-\ell_{2}+\ell_{3} \\
\ell^{\prime}-1
\end{array}\right) \\
& +y \beta_{x} \sum_{\ell_{3}=1}^{\ell}\left(\begin{array}{c}
\ell \\
\ell_{3}-1
\end{array}\right)\left(\begin{array}{l}
\ell \\
\ell_{3}
\end{array}\right) y^{\ell-\ell_{3}} \sum_{\ell_{3}=1}^{\ell^{\prime}}\left(\begin{array}{c}
\ell^{\prime} \\
\ell_{3}-1
\end{array}\right)\left(\begin{array}{l}
\ell^{\prime} \\
\ell_{3}
\end{array}\right) y^{\ell^{\prime}-\ell_{3}}, \quad \ell, \ell^{\prime}=1, \ldots, L .
\end{aligned}
$$

In practice, $y$ may be replaced by $y_{n-1}$. Corollary 3.3 can be used for many high dimensional testing problems and its proof is given in the appendix.

\section{Applications}

In this section, we propose two applications of Theorem 3.1. The first application is about testing the structure of a high-dimensional covariance matrix. The second application applies such structure testing to the analysis of a large fMRI dataset.

\subsection{Testing high dimensional covariance structure}

Testing the structure of high dimensional covariance matrices is a fundamental problem in multivariate and high-dimensional data analysis (Srivastava, 2005; Bai et al. , 2009; Chen, Zhang and Zhong, 2010; Wang and Yao, 2013). Most of this literature consider samples of the form $\mathbf{y}_{i}=\mathbf{Q} \mathbf{x}_{i}$ where the dimension $k$ of the $\mathbf{x}_{i}{ }^{\prime}$ 's is finite. When $k=\infty$ as for time series observations, these existing results are not applicable anymore for structure testing on high dimensional covariance matrices.

We consider two testing problems on high dimensional covariance structure. Let $\Sigma_{0}$ be a given covariance matrix. The first testing problem is given by

$$
H_{01}: \mathrm{QQ}^{*}=\Sigma_{0}
$$

We consider $\operatorname{tr}\left(\boldsymbol{\Sigma}_{0}^{-1 / 2} \mathbf{B}_{n} \boldsymbol{\Sigma}_{0}^{-1 / 2}-\mathbf{I}_{p}\right)^{2}$ as our test statistic, where $\mathbf{B}_{n}=(n-1)^{-1} \sum_{i=1}^{n}\left(\mathbf{y}_{i}-\right.$ $\overline{\mathbf{y}})\left(\mathbf{y}_{i}-\overline{\mathbf{y}}\right)^{T}$. Because $\boldsymbol{\Sigma}_{0}^{-1 / 2} \mathbf{Q} \mathbf{Q}^{*} \boldsymbol{\Sigma}_{0}^{-1 / 2}=\mathbf{I}_{p}$ under $H_{01}, H_{n}=\delta_{1}$ (Dirac mass at 1 ). Therefore, it follows from Corollary 3.3 and the delta method that we have under $H_{01}$

$$
\frac{1}{2}\left\{\operatorname{tr}\left(\boldsymbol{\Sigma}_{0}^{-1} \mathbf{B}_{n}-\mathbf{I}_{p}\right)^{2}-p y_{n-1}-\left(\beta_{x}+1\right) y_{n-1}\right\} / \sqrt{y_{n-1}^{2}+\left(\beta_{x}+2\right) y_{n-1}^{3}} \Longrightarrow N(0,1) .
$$

The second testing problem we consider is about the hypothesis

$$
H_{02}: \mathbf{Q Q}^{*}=\sigma^{2} \boldsymbol{\Sigma}_{0}
$$


When $\boldsymbol{\Sigma}_{0}=\mathbf{I}_{p}$, this reduces to the well known sphericity test (Wang and Yao, 2013). We consider $\operatorname{tr}\left\{\boldsymbol{\Sigma}_{0}^{-1 / 2} \mathbf{B}_{n} \boldsymbol{\Sigma}_{0}^{-1 / 2} /\left[p^{-1} \operatorname{tr}\left(\boldsymbol{\Sigma}_{0}^{-1} \mathbf{B}_{n}\right)\right]-\mathbf{I}_{p}\right\}^{2}$ as our test statistic. Since $\sigma^{-2} \boldsymbol{\Sigma}_{0}^{-1 / 2} \mathbf{Q}$ $\mathbf{Q}^{*} \boldsymbol{\Sigma}_{0}^{-1 / 2}=\mathbf{I}_{p}$ under $H_{02}$, again $H_{n}=\delta_{1}$. By Corollary 3.3 and the delta method, we have under $H_{02}$,

$$
\frac{1}{2}\left\{\operatorname{tr}\left[\boldsymbol{\Sigma}_{0}^{-1} \mathbf{B}_{n} /\left(p^{-1} \operatorname{tr}\left(\boldsymbol{\Sigma}_{0}^{-1} \mathbf{B}_{n}\right)\right)-\mathbf{I}_{p}\right]^{2}-p y_{n-1}-\left(\beta_{x}+1\right) y_{n-1}\right\} / y_{n-1} \Longrightarrow N(0,1) .
$$

Simulation experiments are done to evaluate the finite sample performance of our test for the null $H_{02}$. The test size is set at $5 \%$ and empirical sizes and powers are obtained using 5000 independent replications. We draw $\mathbf{y}_{i}$ according to the $\operatorname{AR}(2)$ model $y_{t i}=$ $\phi_{1} y_{t-1, i}+\phi_{2} y_{t-2, i}+e_{t i}$, where the $e_{t i}$ 's are i.i.d. as $N\left(0, \sigma^{2}\right)$. We set $n \in\{100,200,300\}$ and $p \in\{50,100,200,500,1000\}$. Empirical sizes are evaluated for $\phi_{1} \in\{0.3,0.6\}, \phi_{2} \in$ $\{0.2,0.3\}$, and $\Sigma_{0}=\left\{\gamma_{|i-j|}\right\}_{i, j=1}^{p}$ with $\gamma_{0}=1, \gamma_{1}=\phi_{1} /\left(1-\phi_{2}\right)$ and $\gamma_{\ell}=\phi_{1} \gamma_{\ell-1}+\phi_{2} \gamma_{\ell-2}$ for $\ell \geq 2$. They are given in Table 1. To evaluate empirical powers, the null hypothesis has $\phi_{1}=\phi_{2}=0.18$ and the alternative hypothesis has $\phi_{1} \in\{0.3,0.35\}$ and $\phi_{2} \in\{0.2,0.25\}$. Table 2 presents these empirical powers. Both tables show a very satisfactory finite-sample performance of the proposed test.

Table 1: Empirical test sizes for $H_{02}$ (in percentage)

\begin{tabular}{cc|ccccc|ccccc}
\hline & & \multicolumn{6}{|c|}{$\phi_{1}=0.3$} & \multicolumn{5}{c}{$\phi_{1}=0.6$} \\
$\phi_{2}$ & $n$ & $p=50$ & 100 & 200 & 500 & 1000 & $\mathrm{p}=50$ & 100 & 200 & 500 & 1000 \\
\hline 0.2 & 100 & 5.36 & 5.38 & 5.38 & 5.32 & 5.54 & 5.12 & 5.36 & 5.92 & 5.46 & 4.94 \\
& 200 & 5.12 & 5.46 & 5.22 & 5.22 & 5.80 & 5.76 & 5.34 & 5.32 & 5.14 & 4.76 \\
& 300 & 5.12 & 4.82 & 5.10 & 5.50 & 4.90 & 5.44 & 5.22 & 5.36 & 5.52 & 5.10 \\
\hline 0.3 & 100 & 5.72 & 5.28 & 5.60 & 5.22 & 5.90 & 4.90 & 5.76 & 5.42 & 5.06 & 5.28 \\
& 200 & 4.60 & 5.12 & 5.28 & 5.02 & 5.16 & 5.52 & 5.68 & 5.48 & 5.18 & 5.20 \\
& 300 & 5.04 & 5.56 & 5.30 & 5.24 & 4.88 & 5.54 & 5.64 & 5.48 & 5.50 & 5.56 \\
\hline
\end{tabular}

\subsection{Application to a fMRI dataset}

We apply the proposed method to a structure testing problem for the resting-state fMRI data from the ADHD-200 sample. The whole data was collected from eight sites of the ADHD-200 consortium. We focus on the data from New York University (NYU) with the largest number of subjects. Among them, we used the 1000 ROI extracted time courses 
Table 2: Empirical powers for $H_{02}$ (in percentage)

\begin{tabular}{cc|cccc|cccc}
\hline & & \multicolumn{6}{|c|}{$\phi_{1}=0.3$} & \multicolumn{4}{c}{$\phi_{1}=0.35$} \\
$\phi_{2}$ & $n$ & $p=50$ & 100 & 200 & 500 & $\mathrm{p}=50$ & 100 & 200 & 500 \\
\hline 0.2 & 100 & 59.00 & 61.24 & 62.80 & 64.50 & 98.54 & 99.36 & 99.54 & 100.00 \\
& 200 & 97.98 & 98.90 & 99.28 & 100.00 & 100.00 & 100.00 & 100.00 & 100.00 \\
& 300 & 100.00 & 100.00 & 100.00 & 100.00 & 100.00 & 100.00 & 100.00 & 100.00 \\
\hline 0.25 & 100 & 94.46 & 97.18 & 97.46 & 98.3 & 100.00 & 100.00 & 100.00 & 100.00 \\
& 200 & 100.00 & 100.00 & 100.00 & 100.00 & 100.00 & 100.00 & 100.00 & 100.00 \\
& 300 & 100.00 & 100.00 & 100.00 & 100.00 & 100.00 & 100.00 & 100.00 & 100.00 \\
\hline
\end{tabular}

preprocessed by the Neuroimaging Analysis Kit (NIAK) (Ahn, 2015). The data set consists of 70 subjects $(n=70)$, each of which contains 924 Regions Of Interest (ROIs). Moreover, each ROI contains a time series with 172 time points. At a given ROI, the fMRI observations for the $i$ th subject are treated as $\mathbf{y}_{i}$, a time series of length $p=172$.

The question of interest here is to test whether the standard autoregressive assumption (e.g., $\mathrm{AR}(1)$ or $\mathrm{AR}(2)$ ) is valid for the processed fMRI time series across all ROIs (Lindqusit, 2008). Applying results derived in the previous section, we first test the AR(1) structure with the hypothesis

$$
H_{0} \text { : The time series in a specific ROI is an } \mathrm{AR}(1) \text { process. }
$$

Let $\phi$ be the coefficient of the $\mathrm{AR}(1)$ process and $\boldsymbol{\Sigma}_{\phi}=\left(\phi^{|i-j|}\right)_{i, j=1}^{p}$. The auto-covariance matrix of the $\operatorname{AR}(1)$ process is given by $\mathbf{Q Q}^{*}=\sigma^{2} \boldsymbol{\Sigma}_{\phi}$ and then we have $\operatorname{Cov}\left(\boldsymbol{\Sigma}_{\phi}^{-1 / 2} \mathbf{y}_{i}\right)=$ $\sigma^{2} \mathbf{I}_{p}$, where $\sigma^{2}$ is the variance. Testing the $\mathrm{AR}(1)$ assumption of a given ROI is equivalent to testing the sphericity covariance structure of such ROI. Similar to the arguments of Section 4.1, we can show that (4.4) still holds here. We detail results for ROI 200 and 500. Since $\phi$ takes values in the interval $(-1,1)$, we set $\phi$ be $-1+0.001,-1+0.002, \ldots, 1-0.001$ and calculated the P-values of the test (4.5) in Figure 1, It indicates that both sets of Pvalues are much smaller than $5 \%$, which yields the rejection of the proposed $\mathrm{AR}(1)$ structure in both ROIs.

Next we test the $\operatorname{AR}(2)$ hypothesis

$$
H_{0} \text { : The time series in a given } \mathrm{ROI} \text { is an } \mathrm{AR}(2) \text { process. }
$$

Let $\phi_{1}$ and $\phi_{2}$ be the auto-regressive coefficients of the $\operatorname{AR}(2)$ model. Then, the autocovariance matrix of the $\mathrm{AR}(2)$ model is equal to $\mathbf{Q Q}^{*}=\sigma^{2} \boldsymbol{\Sigma}_{\phi_{1}, \phi_{2}}$, where $\boldsymbol{\Sigma}_{\phi_{1}, \phi_{2}}=$ 

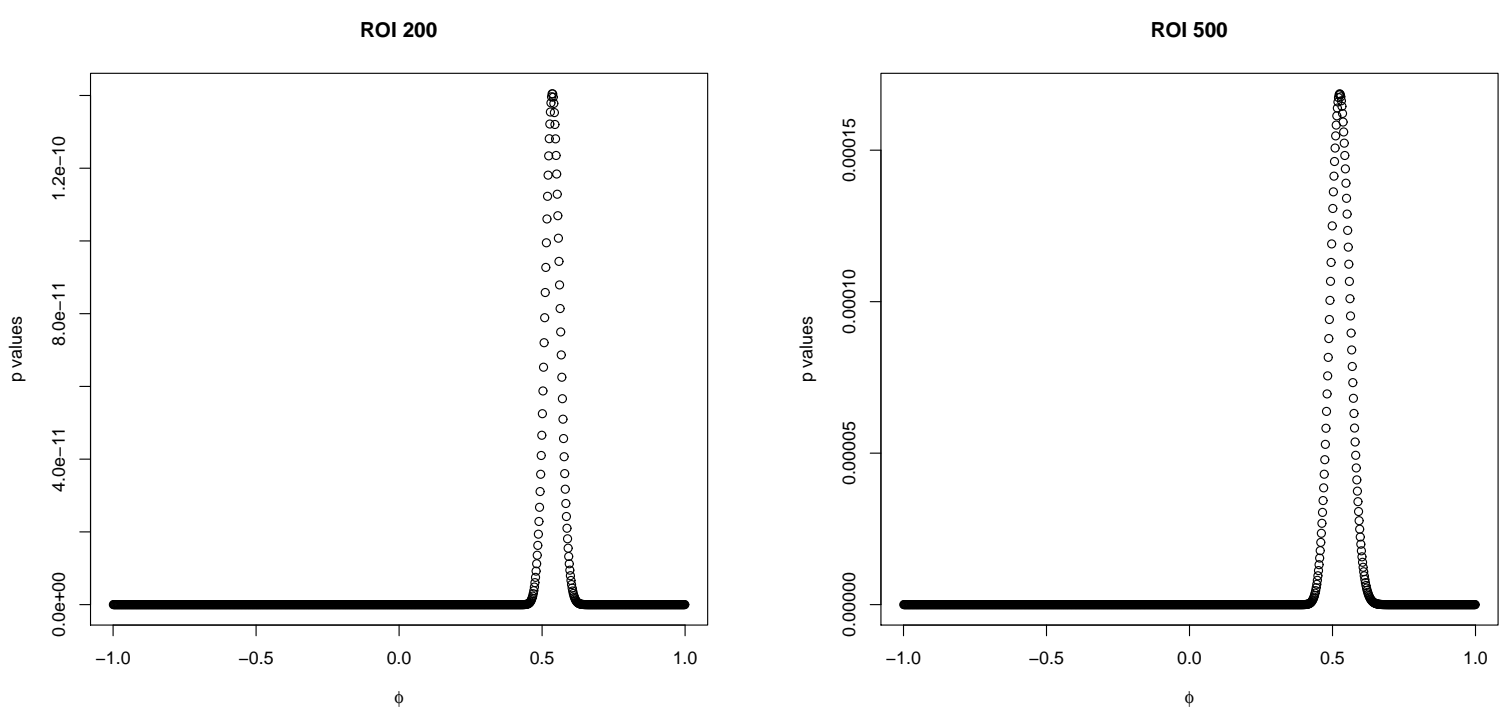

Figure 1: P-values of both ROI 200 and 500 for the testing problem (4.5).

$\left(\gamma_{|i-j|}\right)_{i, j=1}^{p}$ with $\gamma_{0}=1, \gamma_{1}=\phi_{1} /\left(1-\phi_{2}\right)$, and $\gamma_{\ell}=\phi_{1} \gamma_{\ell-1}+\phi_{2} \gamma_{\ell-2}$ for $\ell \geq 2$ satisfying $\phi_{1}^{2}+\phi_{2}^{2}<1$ and $\phi_{2}+\left|\phi_{1}\right|<1$. Then, we have $\operatorname{Cov}\left(\boldsymbol{\Sigma}_{\phi_{1}, \phi_{2}}^{-1 / 2} \mathbf{y}_{i}\right)=\sigma^{2} \mathbf{I}_{p}$. For a specific ROI, testing the $\mathrm{AR}(2)$ assumption is again equivalent to testing the sphericity covariance structure; in particular (4.4) still holds here. Similar to the previous test, we calculated the P-values of our test statistic for every $\phi_{1}, \phi_{2} \in\{-1+0.001,-1+0.002, \ldots, 1-0.001\}$ satisfying $\phi_{1}^{2}+\phi_{2}^{2}<1$ and $\phi_{2}+\left|\phi_{1}\right|<1$ for ROI 200 and 500. As a result, these P-values from both ROI 200 and 500 are much smaller than the nominal level 5\% so that the proposed $A R(2)$ structure is clearly rejected. In summary, neither of the $\operatorname{AR}(1)$ and $\operatorname{AR}(2)$ structures is suitable for the resting-state fMRI data considered here.

\section{Proofs of the main theorems}

\subsection{Proof of Theorem 2.1}

\subsubsection{Case where $Q$ has an infinite number of columns}

Consider a sequence of $\left\{k_{p}\right\}$ that satisfies $p^{-1} \sum_{i=1}^{p} \sum_{\ell=k_{p}+1}^{\infty}\left|q_{i \ell}^{2}\right|=o\left(p^{-2}\right)$. For simplicity, we write $k$ for $k_{p}$. If $\mathbf{Q}$ is a $p \times \infty$ dimensional matrix, then we truncate $\mathbf{Q}$ as $\mathbf{Q}=(\widehat{\mathbf{Q}}, \widetilde{\mathbf{Q}})$ where $\widehat{\mathbf{Q}}$ is a $p \times k$ dimensional matrix and $\widetilde{\mathbf{Q}}=\left(q_{i j}\right)$ is a $p \times \infty$ dimensional matrix with $i=1, \ldots, p, j=k+1, \ldots, \infty$. Similarly, truncate $\mathbf{X}_{n}$ as $\mathbf{X}_{n}=\left(\widehat{\mathbf{X}}_{n}, \widetilde{\mathbf{X}}_{n}\right)$ where $\widehat{\mathbf{X}}_{n}$ is $k \times n$ 
dimensional and $\widetilde{\mathbf{X}}_{n}$ is $\infty \times n$ dimensional. Then we have

$$
L^{4}\left(F^{n^{-1}} \mathbf{Q} \mathbf{X}_{n} \mathbf{X}_{n}^{*} \mathbf{Q}^{*}, F^{n^{-1}} \widehat{\mathbf{Q}}_{n} \widehat{\mathbf{X}}_{n}^{*} \widehat{\mathbf{Q}}^{*}\right) \leq 2 p^{-2} n^{-2} \operatorname{tr}\left(\mathbf{Q} \mathbf{X}_{n} \mathbf{X}_{n}^{*} \mathbf{Q}^{*}+\widehat{\mathbf{Q}} \widehat{\mathbf{X}}_{n} \widehat{\mathbf{X}}_{n}^{*} \widehat{\mathbf{Q}}^{*}\right) \operatorname{tr} \widetilde{\mathbf{Q}} \widetilde{\mathbf{X}}_{n} \widetilde{\mathbf{X}}_{n}^{*} \widetilde{\mathbf{Q}}^{*}
$$

where $L($,$) is the Levy distance, F^{n^{-1}} \mathbf{Q} \mathbf{X}_{n} \mathbf{X}_{n}^{*} \mathbf{Q}^{*}$ is the ESD of $n^{-1} \mathbf{Q} \mathbf{X}_{n} \mathbf{X}_{n}^{*} \mathbf{Q}^{*}$ and $F^{n^{-1}} \widehat{\mathbf{Q}}_{n} \widehat{\mathbf{X}}_{n}^{*} \widehat{\mathbf{Q}}^{*}$ is the ESD of $n^{-1} \widehat{\mathbf{Q}} \widehat{\mathbf{X}}_{n} \widehat{\mathbf{X}}_{n}^{*} \widehat{\mathbf{Q}}^{*}$. We have

$$
\begin{aligned}
& \frac{1}{p n} \operatorname{tr}\left(\widetilde{\mathbf{Q}} \widetilde{\mathbf{X}}_{n} \widetilde{\mathbf{X}}_{n}^{*} \widetilde{\mathbf{Q}}^{*}\right)=\frac{1}{p n} \sum_{i=1}^{p} \sum_{j=1}^{n}\left|\sum_{\ell=k+1}^{\infty} q_{i \ell} x_{\ell j}\right|^{2} \\
= & \frac{1}{p n} \sum_{i=1}^{p} \sum_{j=1}^{n} \sum_{\ell=k+1}^{\infty}\left|q_{i \ell}^{2}\right|\left|x_{\ell j}^{2}\right|+\frac{1}{p n} \sum_{i=1}^{p} \sum_{j=1}^{n} \sum_{k_{1} \neq k_{2}} q_{i k_{1}} \bar{q}_{i k_{2}} x_{k_{1}, j} \bar{x}_{k_{2}, j} .
\end{aligned}
$$

Note that

$$
\mathrm{E}\left(\frac{1}{p n} \sum_{i=1}^{p} \sum_{j=1}^{n} \sum_{\ell=k+1}^{\infty}\left|q_{i k}^{2}\right|\left|x_{\ell j}^{2}\right|\right)=\frac{1}{p} \sum_{i=1}^{p} \sum_{\ell=k+1}^{\infty}\left|q_{i \ell}^{2}\right|=o\left(p^{-2}\right)
$$

and

$$
\begin{aligned}
& \mathrm{E}\left(\frac{1}{p n} \sum_{i=1}^{p} \sum_{j=1}^{n} \sum_{\ell=k+1}^{\infty}\left|q_{i \ell}^{2}\right|\left(\left|x_{\ell j}^{2}\right|-\mathrm{E}\left|x_{\ell j}^{2}\right|\right)\right)^{4} \\
\leq & \frac{1}{p^{4} n^{4}} \sum_{j=1}^{n} \sum_{\ell=k+1}^{\infty}\left(\sum_{i=1}^{p}\left|q_{i \ell}^{2}\right|\right)^{4} \mathrm{E}\left|x_{\ell j}^{8}\right|+\frac{3}{p^{4} n^{4}}\left(\sum_{j=1}^{n} \sum_{\ell=k+1}^{\infty}\left(\sum_{i=1}^{p}\left|q_{i \ell}^{2}\right|\right)^{2} \mathrm{E}\left|x_{\ell j}^{4}\right|\right)^{2} \\
\leq & \frac{\eta_{n}^{6}}{p^{4}} \sum_{\ell=k+1}^{\infty}\left\|\mathbf{q}_{\ell}\right\|^{2}+\frac{3 \eta_{n}^{4}}{p^{4}}\left(\sum_{\ell=k+1}^{\infty}\left\|\mathbf{q}_{\ell}\right\|^{2}\right)^{2}=o\left(p^{-2}\right) .
\end{aligned}
$$

These inequalities simply imply $(p n)^{-1} \sum_{i=1}^{p} \sum_{j=1}^{n} \sum_{\ell=1}^{k}\left|q_{i \ell}^{2}\right|\left|x_{\ell j}^{2}\right|=o_{a . s .}$ (1). Furthermore,

$$
\begin{aligned}
& \mathrm{E}\left(\frac{1}{p n} \sum_{i=1}^{p} \sum_{j=1}^{n} \sum_{k_{1} \neq k_{2}} q_{i k_{1}} \bar{q}_{i k_{2}} x_{k_{1}, j} \bar{x}_{k_{2}, j}\right)^{2} \leq \frac{2}{p^{2} n^{2}} \sum_{j=1}^{n} \sum_{k_{1} \neq k_{2}}\left|\sum_{i=1}^{p} q_{i k_{1}} \bar{q}_{i k_{2}}\right|^{2} \\
\leq & \frac{2}{p^{2} n} \operatorname{tr}\left(\widetilde{\mathbf{Q}} \widetilde{\mathbf{Q}}^{*}\right)^{2}=o\left(p^{-2}\right),
\end{aligned}
$$

which implies that $(p n)^{-1} \sum_{i=1}^{p} \sum_{j=1}^{n} \sum_{k_{1} \neq k_{2}} q_{i k_{1}} \bar{q}_{i k_{2}} x_{k_{1}, j} \bar{x}_{k_{2}, j} \rightarrow 0$,a.s. Then we have

$$
(p n)^{-1} \operatorname{tr}\left(\widetilde{\mathbf{Q}} \widetilde{\mathbf{X}}_{n} \widetilde{\mathbf{X}}_{n}^{*} \widetilde{\mathbf{Q}}^{*}\right)=o_{a . s .}(1) .
$$

Similarly, we can prove $(p n)^{-1} \operatorname{tr}\left(\mathbf{Q} \mathbf{X}_{n} \mathbf{X}_{n}^{*} \mathbf{Q}^{*}\right)=O_{a . s .}(1)$ and $(p n)^{-1} \operatorname{tr}\left(\widehat{\mathbf{Q}} \widehat{\mathbf{X}}_{n} \widehat{\mathbf{X}}_{n}^{*} \widehat{\mathbf{Q}}^{*}\right)=O_{\text {a.s. }}(1)$. Then we have

$$
L^{4}\left(F^{n^{-1}} \mathbf{Q} \mathbf{X}_{n} \mathbf{X}_{n}^{*} \mathbf{Q}^{*}, F^{n^{-1}} \widehat{\mathbf{Q}} \widehat{\mathbf{X}}_{n} \widehat{\mathbf{X}}_{n}^{*} \widehat{\mathbf{Q}}^{*}\right)=o_{a . s .}(1) .
$$

Therefore, without loss of generality, we will hereafter assume that the number of columns $k$ of $\mathbf{Q}$ is finite. 


\subsubsection{Sketch of the proof of Theorem 2.1}

Silverstein (1995) obtained the LSD of the classical sample covariance matrix when the dimension increases proportionally with the sample size. But the sample covariance matrix $\mathbf{B}_{n}$ of this paper is different from that of Silverstein (1995) because Silverstein (1995) assumed that $\mathbf{Q}$ is $p \times p$ and nonnegative definite while this paper assumes $\mathbf{Q}$ is $p \times k$ with $k \geq p$, even being infinity. In establishing the LSD of $\mathbf{B}_{n}$ under the assumptions of Theorem 2.1, we need the truncation, centralization and rescaling on $x_{i j}$. Under the assumptions of Theorem 2.1, the LSD of $\mathbf{B}_{n}$ will be the same before or after the truncation, centralization and rescaling. Because the dimension of $\mathbf{Q}$ is different from those defined in Silverstein (1995), the assumptions and truncations of Theorem 2.1 are also different from those given in Silverstein (1995). The readers are reminded that the corresponding proofs given in Bai and Silverstein (2010) and Silverstein (1995), strongly depend on a fact that

$\sum_{j=1}^{n} \beta_{j}(z)=\operatorname{tr}\left(n^{-1} \mathbf{X}_{n}^{*} \widetilde{\mathbf{T}}_{n} \mathbf{X}_{n}-z \mathbf{I}_{n}\right)^{-1}$, where $\beta_{j}(z)$ 's are defined in Subsection 5.1 .3 and $\widetilde{\mathbf{T}}_{n}=\mathbf{T}_{n}$ in Silverstein (1995) while $\widetilde{\mathbf{T}}_{n}=\mathbf{Q}^{*} \mathbf{Q}$ in the present paper. It may cause some ambiguity when $k$ is infinity in the multiplication of infinite-dimensional matrices, we will avoid to use this fact in this paper.

Let $m_{n}(z)=p^{-1} \operatorname{tr}\left(\mathbf{B}_{n}-z \mathbf{I}_{p}\right)^{-1}$ be the Stieltjes transform of the ESD of $\mathbf{B}_{n}$, where $z=u+i v$ with $v>0$. We will show that $\underline{m}_{n}(z)$ tends to a non-random limit that satisfies (2.2), where $\underline{m}_{n}(z)=-\left(1-y_{n}\right) z^{-1}+y_{n} m_{n}(z)$ is in fact the Stieltjes transform of $\underline{\mathbf{B}}_{n}=n^{-1} \mathbf{X}_{n}^{*} \widetilde{\mathbf{T}}_{n} \mathbf{X}_{n}$, where $\widetilde{\mathbf{T}}_{n}=\mathbf{Q}^{*} \mathbf{Q}$. The proof will be split into two parts:

$$
\begin{aligned}
& \text { (i) } \quad m_{n}(z)-\mathrm{E} m_{n}(z) \rightarrow 0 \text { a.s., } \\
& \text { (ii) } \quad \operatorname{E} \underline{m}_{n}(z) \rightarrow \underline{m}(z) \text { satisfying (2.2). }
\end{aligned}
$$

For truncation and re-normalization (see Appendix B), we may further assume $\left|x_{i j}\right|<$ $\eta_{n} \sqrt{n} /\left\|\mathbf{q}_{i}\right\|$, where the constant sequence $\left\{\eta_{n}\right\}$ tends to zero as $n \rightarrow \infty$.

\subsubsection{Proof of (5.3)}

Let $\mathbf{r}_{j}=n^{-1 / 2} \mathbf{Q} \mathbf{x}_{j}$, then $\mathbf{B}_{n}=\sum_{j=1}^{n} \mathbf{r}_{j} \mathbf{r}_{j}^{*}$. Define $\mathbf{D}(z)=\mathbf{B}_{n}-z \mathbf{I}_{p}$ and

$$
\mathbf{D}_{j}(z)=\mathbf{D}(z)-\mathbf{r}_{j} \mathbf{r}_{j}^{*}, \quad \beta_{j}(z)=\left[1+\mathbf{r}_{j}^{*} \mathbf{D}_{j}^{-1}(z) \mathbf{r}_{j}\right]^{-1}, \quad \gamma_{j}(z)=\mathbf{r}_{j}^{*} \mathbf{D}_{j}^{-2}(z) \mathbf{r}_{j} \beta_{j}(z) .
$$

Since $\Im\left(\beta_{j}^{-1}(z)\right)=v \mathbf{r}_{j}^{*} \mathbf{D}_{j}^{-1}(z)\left(\mathbf{D}_{j}^{-1}(z)\right)^{*} \mathbf{r}_{j}>v\left|\mathbf{r}_{j}^{*} \mathbf{D}_{j}^{-2}(z) \mathbf{r}_{j}\right|$, we obtain

$$
\left|\gamma_{j}(z)\right| \leq v^{-1},
$$


where $\Im(z)=v$. Moreover,

$$
\begin{aligned}
\Im\left[z \mathbf{r}_{j}^{*} \mathbf{D}_{j}^{-1}(z) \mathbf{r}_{j}\right] & =(2 \mathbf{i})^{-1}\left[z \mathbf{r}_{j}^{*} \mathbf{D}_{j}^{-1}(z) \mathbf{r}_{j}-\bar{z} \mathbf{r}_{j}^{*} \overline{\mathbf{D}_{j}^{-1}}(z) \mathbf{r}_{j}\right] \\
& =v|z|^{-2} z \mathbf{r}_{j}^{*} \mathbf{D}_{j}^{-1}(z)\left(\sum_{i \neq j} \bar{z} \mathbf{r}_{i} \mathbf{r}_{i}^{*}\right) \overline{\mathbf{D}_{j}^{-1}}(z) \mathbf{r}_{j} \geq 0
\end{aligned}
$$

where $\bar{z}$ and $\overline{\mathbf{D}_{j}^{-1}}(z)$ denote the conjugate of $z$ and $\mathbf{D}_{j}^{-1}(z)$. Thus we have

$$
\left|\beta_{j}(z)\right| \leq|z| v^{-1}
$$

Denote the conditional expectation given $\left\{\mathbf{r}_{1}, \cdots, \mathbf{r}_{j}\right\}$ by $\mathrm{E}_{j}$ and $\mathrm{E}_{0}$ for the unconditional expectation. Then, we have

$$
\begin{aligned}
m_{n}(z)-\mathrm{Em}_{n}(z) & =p^{-1} \sum_{j=1}^{n}\left(\mathrm{E}_{j}-\mathrm{E}_{j-1}\right) \operatorname{tr} \mathbf{D}^{-1}(z) \\
& =p^{-1} \sum_{j=1}^{n}\left(\mathrm{E}_{j}-\mathrm{E}_{j-1}\right)\left[\operatorname{tr} \mathbf{D}^{-1}(z)-\operatorname{tr} \mathbf{D}_{j}^{-1}(z)\right] \\
& =p^{-1} \sum_{j=1}^{n}\left(\mathrm{E}_{j}-\mathrm{E}_{j-1}\right) \boldsymbol{\gamma}_{j}(z)
\end{aligned}
$$

By Lemma A.1 (Burkholder inequality) and the inequality (5.6) , for any $\ell>1$, we obtain

$$
\mathrm{E}\left|m_{n}(z)-\mathrm{E}_{n}(z)\right|^{\ell} \leq K_{0} p^{-\ell} \mathrm{E}\left(\sum_{j=1}^{n}\left|\left(\mathrm{E}_{j}-\mathrm{E}_{j-1}\right) \gamma_{j}(z)\right|^{2}\right)^{\frac{\ell}{2}} \leq K_{0} v^{-\ell} p^{-\ell} n^{\frac{\ell}{2}}
$$

where $K_{0}$ is a constant. Taking $\ell>2$, Chebyshev inequality and (5.9) imply (5.3) $: m_{n}(z)-$ $\mathrm{Em}_{n}(z) \rightarrow 0$ a.s.

\subsubsection{Proof of (5.4)}

Following the steps of the proof of Theorem 1.1 of Bai and Zhou (2008), define $\mathbf{K}=$ $\left(1+y_{n} a_{n, 1}\right)^{-1} \mathbf{T}_{n}$ and $y_{n}=p / n$, where $\mathbf{T}_{n}=\mathbf{Q} \mathbf{Q}^{*}, a_{n, \ell}=p^{-1} \operatorname{Etr}\left[\mathbf{T}_{n}^{\ell} \mathbf{D}^{-1}(z)\right], \ell=0$ or 1. We have

$$
\begin{aligned}
(\mathbf{K}-z \mathbf{I})^{-1}-\mathbf{D}^{-1}(z) & =\sum_{j=1}^{n}(\mathbf{K}-z \mathbf{I})^{-1} \mathbf{r}_{j} \mathbf{r}_{j}^{*} \mathbf{D}^{-1}(z)-(\mathbf{K}-z \mathbf{I})^{-1} \mathbf{K} \mathbf{D}^{-1}(z) \\
& =\sum_{j=1}^{n}(\mathbf{K}-z \mathbf{I})^{-1} \mathbf{r}_{j} \mathbf{r}_{j}^{*} \mathbf{D}_{j}^{-1}(z) \beta_{j}(z)-(\mathbf{K}-z \mathbf{I})^{-1} \mathbf{K D}^{-1}(z) .
\end{aligned}
$$


For $\ell=0,1$, multiplying both sides by $\mathbf{T}_{n}^{\ell}$ and then taking trace and dividing by $p$, we have

$$
\begin{aligned}
& p^{-1} \operatorname{Etr}\left[\mathbf{T}_{n}^{\ell}(\mathbf{K}-z \mathbf{I})^{-1}\right]-a_{n, \ell} \\
= & p^{-1} \sum_{j=1}^{n} \operatorname{Er}_{j}^{*} \mathbf{D}_{j}^{-1}(z) \mathbf{T}_{n}^{\ell}(\mathbf{K}-z \mathbf{I})^{-1} \mathbf{r}_{j} \beta_{j}(z)-p^{-1} \operatorname{Etr}\left[\mathbf{T}_{n}^{\ell}(\mathbf{K}-z \mathbf{I})^{-1} \mathbf{K D}^{-1}(z)\right](5,11
\end{aligned}
$$

One can prove a formula similar to (1.15) of Bai and Silverstein (2004) and verify that

$$
\begin{aligned}
& \mathrm{E}\left|\mathbf{r}_{j}^{*} \mathbf{D}_{j}^{-1}(z) \mathbf{r}_{j}-n^{-1} \operatorname{tr}\left(\mathbf{Q}^{*} \mathbf{D}_{j}^{-1}(z) \mathbf{Q}\right)\right|^{2} \\
\leq & 2 n^{-2} \operatorname{Etr}\left(\mathbf{Q}^{*} \mathbf{D}_{j}^{-1}(z) \mathbf{Q}\right)\left(\mathbf{Q}^{*} \mathbf{D}_{j}^{-1}(z) \mathbf{Q}\right)^{*}+n^{-2} \sum_{i=1}^{k}\left|\left(\mathbf{Q}^{*} \mathbf{D}_{j}^{-1}(z) \mathbf{Q}\right)_{i i}\right|^{2} \mathrm{E}\left|x_{i j}^{4}\right| \\
\leq & 2 n^{-2} p v^{-2}\|\mathbf{Q}\|^{4}+n^{-2} v^{-2} \sum_{i=1}^{k}\left\|\mathbf{q}_{i}\right\|^{4} \eta_{n}^{2} n /\left\|\mathbf{q}_{i}\right\|^{2} \leq C \eta_{n}^{2} \rightarrow 0 .
\end{aligned}
$$

By Lemma A.7, one can prove that

$$
\left|n^{-1} \operatorname{tr}\left(\mathbf{Q}^{*} \mathbf{D}_{j}^{-1}(z) \mathbf{Q}\right)-n^{-1} \operatorname{tr}\left(\mathbf{Q}^{*} \mathbf{D}^{-1}(z) \mathbf{Q}\right)\right|^{2} \leq K\left(n^{2} v^{2}\right)^{-1}
$$

and by the similar method of the proof of (5.3), we have

$$
\mathrm{E}\left|n^{-1} \operatorname{tr}\left(\mathbf{Q}^{*} \mathbf{D}^{-1}(z) \mathbf{Q}\right)-n^{-1} \operatorname{Etr}\left(\mathbf{Q}^{*} \mathbf{D}^{-1}(z) \mathbf{Q}\right)\right|^{2} \leq K\left(n v^{2}\right)^{-1} .
$$

Therefore we have $\mathrm{E}\left|\beta_{j}^{-1}(z)-\left(1+y_{n} a_{n, 1}\right)\right|^{2}=o(1)$ which, applying Lemma A.7 again and (5.7), implies that

$$
\begin{aligned}
& p^{-1} \sum_{j=1}^{n} \operatorname{Er}_{j}^{*} \mathbf{D}_{j}^{-1}(z) \mathbf{T}_{n}^{\ell}(\mathbf{K}-z \mathbf{I})^{-1} \mathbf{r}_{j} \beta_{j}(z)-p^{-1} \operatorname{Etr}\left[\mathbf{T}_{n}^{\ell}(\mathbf{K}-z \mathbf{I})^{-1} \mathbf{K D}^{-1}(z)\right] \\
= & p^{-1} \sum_{j=1}^{n} \operatorname{Er}_{j}^{*} \mathbf{D}_{j}^{-1}(z) \mathbf{T}_{n}^{\ell}(\mathbf{K}-z \mathbf{I})^{-1} \mathbf{r}_{j}\left(1+y_{n} a_{n, 1}\right)^{-1} \\
& -p^{-1} \operatorname{Etr}\left[\mathbf{T}_{n}^{\ell}(\mathbf{K}-z \mathbf{I})^{-1} \mathbf{K} \mathbf{D}^{-1}(z)\right]+o(1) \\
= & (p n)^{-1} \sum_{j=1}^{n} \operatorname{Etr} \mathbf{D}_{j}^{-1}(z) \mathbf{T}_{n}^{\ell}(\mathbf{K}-z \mathbf{I})^{-1} \mathbf{T}_{n}\left(1+y_{n} a_{n, 1}\right)^{-1} \\
& -p^{-1} \operatorname{Etr}\left[\mathbf{T}_{n}^{\ell}(\mathbf{K}-z \mathbf{I})^{-1} \mathbf{K} \mathbf{D}^{-1}(z)\right]+o(1) \\
= & (p n)^{-1} \sum_{j=1}^{n} \operatorname{Etr}_{j}^{-1}(z) \mathbf{T}_{n}^{\ell}(\mathbf{K}-z \mathbf{I})^{-1} \mathbf{K}-p^{-1} \operatorname{Etr}^{-1}(z) \mathbf{T}_{n}^{\ell}(\mathbf{K}-z \mathbf{I})^{-1} \mathbf{K}+o(1) \\
= & o(1) .
\end{aligned}
$$


It then follows from (5.11) and (5.13) that

$$
\begin{aligned}
a_{n, \ell} & =p^{-1} \operatorname{tr}\left\{\mathbf{T}_{n}^{\ell}\left[\left(1+y_{n} a_{n, 1}\right)^{-1} \mathbf{T}_{n}-z \mathbf{I}\right]^{-1}\right\}+o(1) \\
& =\int \frac{t^{\ell}}{t\left(1+y_{n} a_{n, 1}\right)^{-1}-z} d H_{n}(t)+o(1),
\end{aligned}
$$

where $H_{n}$ is the ESD of $\mathbf{T}_{n}$. Because $\Im\left[z\left(1+y_{n} a_{n, 1}\right)\right]>v$, we conclude that $\left|\left(1+y_{n} a_{n, 1}\right)^{-1}\right| \leq$ $|z| / v$. Taking $\ell=1$ in (5.14) and multiplying both sides by $\left(1+y_{n} a_{n, 1}\right)^{-1}$, we obtain

$$
\begin{aligned}
\frac{a_{n, 1}}{1+y_{n} a_{n, 1}} & =\int \frac{t\left(1+y_{n} a_{n, 1}\right)^{-1}}{t\left(1+y_{n} a_{n, 1}\right)^{-1}-z} d H_{n}(t)+o(1) \\
& =1+z \int \frac{1}{t\left(1+y_{n} a_{n, 1}\right)^{-1}-z} d H_{n}(t)+o(1) \\
& =1+z a_{n, 0}+o(1) .
\end{aligned}
$$

From this, one can easily derive that

$$
\frac{1}{1+y_{n} a_{n, 1}}=1-y_{n}\left(1+z a_{n, 0}\right)+o(1)=1-y_{n}\left[1+z \mathrm{E} m_{n}(z)\right]+o(1) .
$$

Finally, from (5.14) with $\ell=0$, we obtain

$$
\mathrm{E} m_{n}(z)=\int \frac{1}{t\left[1-y_{n}\left(1+z \mathrm{E} m_{n}(z)\right)\right]-z} d H_{n}(t)+o(1) .
$$

The limiting equation of the above estimate is

$$
m(z)=\int \frac{1}{t\left[1-y\left(1+z m_{y}(z)\right)\right]-z} d H(t) .
$$

It was proved in Silverstein (1995) that for each $z \in \mathbb{C}^{+}$the above equation has a unique solution $m(z)$ satisfying $\Im(m)>0$. By this fact, we conclude that $\mathrm{E}_{n}(z)$ tends to the unique solution to the equation (5.17). Finally, by the relation $\underline{m}(z)=-(1-y) z^{-1}+y m(z)$, we obtain (5.4).

\subsection{Proof of Theorem 3.1}

First, when the number of columns $k$ in $\mathbf{Q}$ is infinite we can reduce the situation to the finite $k$ case using arguments analoguous to those developed in Section 5.1.1. Therefore, without loss of generality we assume in this section that $k$ is finite.

\subsubsection{Sketch of the proof of Theorem 3.1}

Let $M_{n}(z)=p\left[m_{n}(z)-m_{n}^{0}(z)\right]=n\left[\underline{m}_{n}(z)-\underline{m}_{n}^{0}(z)\right]$ where $m_{n}(z)$ is the Stieltjes transform of the ESD of $\mathbf{B}_{n}, m_{n}^{0}(z)$ and $\underline{m}_{n}^{0}(z)$ are the Stieltjes transforms of $F^{y_{n}, H_{n}}$ and $\underline{F}^{y_{n}, H_{n}}$ 
satisfying

$$
z=-\frac{1}{\underline{m}_{n}^{0}(z)}+y_{n} \int \frac{t d H_{n}(t)}{1+t \underline{m}_{n}^{0}(z)}
$$

with $y_{n}=p / n$ and $H_{n}$ being the ESD of $\mathbf{T}_{n}$. Let $x_{r}$ be a number greater than $(1+$ $\sqrt{y})^{2} \lim \sup \lambda_{\max }^{\mathbf{T}_{n}}$. Let $x_{l}$ be a number between 0 and $(1-\sqrt{y})^{2} \liminf \lambda_{\min }^{\mathbf{T}_{n}}$ if the latter is greater than 0 and $y<1$. Otherwise, let $x_{l}$ be a negative number. Let $\eta_{l}$ and $\eta_{r}$ satisfy

$$
x_{l}<\eta_{l}<(1-\sqrt{y})^{2} I_{(0<y<1)} \liminf { }_{n} \lambda_{\min }^{\mathbf{T}_{n}}<(1+\sqrt{y})^{2} \lim \sup _{n} \lambda_{\max }^{\mathbf{T}_{n}}<\eta_{r}<x_{r} .
$$

Let $v_{0}$ be any positive number. Define a contour $\mathcal{C}=\left\{x_{l}+i v:|v| \leq v_{0}\right\} \cup \mathcal{C}_{u} \cup \mathcal{C}_{b} \cup\left\{x_{r}+i v\right.$ : $\left.|v| \leq v_{0}\right\}$ where

$$
\mathcal{C}_{u}=\left\{x+i v_{0}: x \in\left[x_{l}, x_{r}\right]\right\}, \quad \mathcal{C}_{b}=\left\{x-i v_{0}: x \in\left[x_{l}, x_{r}\right]\right\}
$$

and $\mathcal{C}_{n}=\left\{z: \quad z \in \mathcal{C}\right.$ and $\left.|\Im(z)|>n^{-1} \epsilon_{n}\right\}$ with $\epsilon_{n} \geq n^{-\alpha}, 0<\alpha<1$. Let $M_{n}(z)=$ $p\left[m_{n}(z)-m_{F^{y_{n}, H_{n}}}(z)\right]$ and

$$
\widehat{M}_{n}(z)= \begin{cases}M_{n}(z), & z \in \mathcal{C}_{n}, \\ M_{n}\left(x_{r}+i n^{-1} \epsilon_{n}\right), & x=x_{r}, v \in\left[0, n^{-1} \epsilon_{n}\right], \\ M_{n}\left(x_{r}-i n^{-1} \epsilon_{n}\right), & x=x_{r}, v \in\left[-n^{-1} \epsilon_{n}, 0\right), \\ M_{n}\left(x_{l}+i n^{-1} \epsilon_{n}\right), & x_{l}<0, x=x_{l},|v| \in\left[0, n^{-1} \epsilon_{n}\right], \\ M_{n}\left(x_{l}-i n^{-1} \epsilon_{n}\right), & x_{l}<0, x=x_{l},|v| \in\left[-n^{-1} \epsilon_{n}, 0\right) .\end{cases}
$$

Lemma 5.1. Under Assumptions (b)-(c)-(d)-(e)-(f), there exists $\left\{\epsilon_{n}\right\}$ for which $\left\{\widehat{M}_{n}(\cdot)\right\}$ forms a tight sequence on $\mathcal{C}$. Moreover, $\widehat{M}_{n}(\cdot)$ converges weakly to a two-dimensional Gaussian process $M(\cdot)$ satisfying for $z \in \mathcal{C}$

$$
\begin{aligned}
\operatorname{EM}(z)= & \alpha_{x} \frac{y \int \underline{m}^{3}(z) t^{2}(1+t \underline{m}(z))^{-3} d H(t)}{\left(1-y \int \frac{\underline{m}^{2}(z) t^{2}}{(1+t \underline{m}(z))^{2}} d H(t)\right)\left(1-\alpha_{x} y \int \frac{\underline{m}^{2}(z) t^{2}}{(1+\underline{\underline{m}}(z))^{2}} d H(t)\right)} \\
& +\beta_{x} \frac{y \int \underline{m^{3}}(z) t^{2}(1+t \underline{m}(z))^{-3} d H(t)}{1-y \int \underline{m}^{2}(z) t^{2}(1+t \underline{m}(z))^{-2} d H(t)},
\end{aligned}
$$

and variance-covariance function

$$
\begin{aligned}
& \operatorname{Cov}\left(M\left(z_{1}\right), M\left(z_{2}\right)\right) \\
= & \frac{\left(\partial \underline{m}\left(z_{1}\right) / \partial z_{1}\right)\left(\partial \underline{m}\left(z_{2}\right) / \partial z_{2}\right)}{\left(\underline{m}\left(z_{1}\right)-\underline{m}\left(z_{2}\right)\right)^{2}}+y \beta_{x}\left[\int \frac{t}{\left(\underline{m}\left(z_{1}\right) t+1\right)^{2}} \frac{t}{\left(\underline{m}\left(z_{2}\right) t+1\right)^{2}} d H(t)\right] \frac{\partial \underline{m}\left(z_{1}\right)}{\partial z_{1}} \frac{\partial \underline{m}\left(z_{2}\right)}{\partial z_{2}} \\
& -\frac{1}{\left(z_{1}-z_{2}\right)^{2}}-\frac{\partial^{2}}{\partial z_{1} \partial z_{2}} \log \left(1-a\left(z_{1}, z_{2}\right)\right)
\end{aligned}
$$

where

$$
a\left(z_{1}, z_{2}\right)=\alpha_{x}\left(1+\frac{\underline{m}\left(z_{1}\right) \underline{m}\left(z_{2}\right)\left(z_{1}-z_{2}\right)}{\underline{m}\left(z_{2}\right)-\underline{m}\left(z_{1}\right)}\right) .
$$


The proof of Lemma 5.1 is given in the following Section 5.2.2. We show how Theorem 3.1 follows from the above Lemma. We use the identity

$$
\int f(x) d G(x)=-\frac{1}{2 \pi \mathbf{i}} \oint_{\mathcal{C}} f(z) m(z) d z
$$

valid for c.d.f. $G$ and $f$ analytic on the support of $G$ where $m(z)$ is the Stieltjes transform of $G$. The complex integral on the right is over any positively oriented contour enclosing the support of $G$ and on which $f$ is analytic. Choose $v_{0}, x_{r}$, and $x_{l}$ so that $f_{1}, \ldots, f_{L}$ are all analytic on and inside the contour $\mathcal{C}$. Therefore for any $f \in\left\{f_{1}, \ldots, f_{L}\right\}$, with probability one $\int f(x) d G_{n}(x)=-(2 \pi \mathbf{i})^{-1} \int f(z) M_{n}(z) d z$ for all $n$ large, where the complex integral is over $\mathcal{C}$. Moreover, with probability one, for all $n$ large

$$
\begin{aligned}
\left|\int f(z)\left(M_{n}(z)-\widehat{M}_{n}(z)\right) d z\right| \leq & 4 K \epsilon_{n}\left(\left|\max \left(\lambda_{\max }^{\mathbf{T}_{n}}\left(1+\sqrt{c_{n}}\right)^{2}, \lambda_{\max }^{\mathbf{B}_{n}}\right)-x_{r}\right|^{-1}\right. \\
& \left.+\left|\min \left(\lambda_{\min }^{\mathbf{T}_{n}} I_{(0,1)}\left(c_{n}\right)\left(1-\sqrt{c_{n}}\right)^{2}, \lambda_{\min }^{\mathbf{B}_{n}}\right)-x_{l}\right|^{-1}\right)
\end{aligned}
$$

which converges to zero as $n \rightarrow \infty$. Here $K$ is a bound on $f$ over $\mathcal{C}$. Since

$$
\widehat{M}_{n}(\cdot) \longrightarrow\left(-\frac{1}{2 \pi \mathbf{i}} \int f_{1}(z) \widehat{M}_{n}(z) d z, \ldots,-\frac{1}{2 \pi \mathbf{i}} \int f_{L}(z) \widehat{M}_{n}(z) d z\right)
$$

is a continuous mapping of $C\left(\mathcal{C}, \mathbb{R}^{2}\right)$ into $\mathbb{R}^{r}$, it follows that the above vector forms tight sequences. Letting $M(\cdot)$ denote the limit of any weakly converging subsequence of $\left\{\widehat{M}_{n}(\cdot)\right\}$, then we have the weak limit equal in distribution to

$$
\left(-\frac{1}{2 \pi \mathbf{i}} \int f_{1}(z) M(z) d z, \ldots,-\frac{1}{2 \pi \mathbf{i}} \int f_{L}(z) M(z) d z\right) .
$$

The fact that this vector, under the assumptions (b)-(c)-(d)-(e)-(f), is multivariate Gaussian following from the fact that Riemann sums corresponding to these integrals are multivariate Gaussian, and that weak limits of Gaussian vectors can only be Gaussian. The limiting expressions for the mean and covariance follow immediately.

\subsubsection{Proof of Lemma 5.1}

The proof of Lemma 5.1 is similar to the proof of Lemma 1.1 of Bai and Silverstein (2004) but the proof of the mean and variance-covariance is different from Lemma 1.1 of Bai and Silverstein (2004). Let $M_{n}(z)=p\left[m_{n}(z)-m_{n}^{0}(z)\right]$. In fact, we also have $M_{n}(z)=n\left[\underline{m}_{n}(z)-\underline{m}_{n}^{0}(z)\right]$.

Convergence of finite dimensional distributions. Write for $z \in \mathcal{C}_{n}, M_{n}(z)=M_{n}^{1}(z)+M_{n}^{2}(z)$ where

$$
M_{n}^{1}(z)=p\left[m_{n}(z)-\mathrm{E} m_{n}(z)\right], \quad M_{n}^{2}(z)=p\left[\operatorname{E} m_{n}(z)-m_{n}^{0}(z)\right],
$$


and $m_{n}^{0}(z)$ is the Stieltjes transform of $F^{y_{n}, H_{n}}$. In this subsection we will show that for any positive integer $r$ and any complex numbers $z_{1}, \cdots, z_{r}$, the random vector $\left(M_{n}^{\ell}\left(z_{j}\right), j=\right.$ $1, \cdots, r)$ converges to an $2 r$-dimensional Gaussian vector for $\ell=1,2$. Because of Assumption (e), without loss of generality, we may assume $\|\mathbf{Q}\| \leq 1$ for all $n$. Constants appearing in inequalities will be denoted by $K$ and may take on different values from one expression to the next. Let

$$
\epsilon_{j}(z)=\mathbf{r}_{j}^{*} \mathbf{D}_{j}^{-1}(z) \mathbf{r}_{j}-n^{-1} \operatorname{tr}_{n} \mathbf{D}_{j}^{-1}(z), \quad \delta_{j}(z)=\mathbf{r}_{j}^{*} \mathbf{D}_{j}^{-2}(z) \mathbf{r}_{j}-n^{-1} \operatorname{tr} \mathbf{T}_{n} \mathbf{D}_{j}^{-2}(z)=\frac{d}{d z} \epsilon_{j}(z),
$$

and

$$
\bar{\beta}_{j}(z)=\frac{1}{1+n^{-1} \operatorname{tr} \mathbf{T}_{n} \mathbf{D}_{j}^{-1}(z)}, \quad b_{n}(z)=\frac{1}{1+n^{-1} \operatorname{Etr}_{n} \mathbf{D}^{-1}(z)} .
$$

Notice that

$$
\mathbf{D}^{-1}(z)=\mathbf{D}_{j}^{-1}(z)-\mathbf{D}_{j}^{-1}(z) \mathbf{r}_{j} \mathbf{r}_{j}^{*} \mathbf{D}_{j}^{-1}(z) \beta_{j}(z) .
$$

By (5.22), we obtain

$$
\begin{aligned}
p\left[m_{n}(z)-\mathrm{Em}_{n}(z)\right] & =\operatorname{tr}\left[\mathbf{D}^{-1}(z)-\mathrm{ED}^{-1}(z)\right] \\
& =\sum_{j=1}^{n} \operatorname{trE}_{j} \mathbf{D}^{-1}(z)-\operatorname{tr}_{j-1} \mathbf{D}^{-1}(z) \\
& =\sum_{j=1}^{n} \operatorname{trE}_{j}\left[\mathbf{D}^{-1}(z)-\mathbf{D}_{j}^{-1}(z)\right]-\operatorname{trE}_{j-1}\left[\mathbf{D}^{-1}(z)-\mathbf{D}_{j}^{-1}(z)\right] \\
& =-\sum_{j=1}^{n}\left(\mathrm{E}_{j}-\mathrm{E}_{j-1}\right) \beta_{j}(z) \mathbf{r}_{j}^{*} \mathbf{D}_{j}^{-2}(z) \mathbf{r}_{j} \\
& =-\frac{d}{d z} \sum_{j=1}^{n}\left(\mathrm{E}_{j}-\mathrm{E}_{j-1}\right) \log \beta_{j}(z) \\
& =\frac{d}{d z} \sum_{j=1}^{n}\left(\mathrm{E}_{j}-\mathrm{E}_{j-1}\right) \log \left(1+\epsilon_{j}(z) \bar{\beta}_{j}(z)\right)
\end{aligned}
$$

where $\beta_{j}(z)=\bar{\beta}_{j}(z)-\beta_{j}(z) \bar{\beta}_{j}(z) \epsilon_{j}(z)$. By Lemma A.2, we have

$$
\begin{aligned}
& \mathrm{E}\left|\frac{d}{d z} \sum_{j=1}^{n}\left(\mathrm{E}_{j}-\mathrm{E}_{j-1}\right)\left[\log \left(1+\epsilon_{j}(z) \bar{\beta}_{j}(z)\right)-\epsilon_{j}(z) \bar{\beta}_{j}(z)\right]\right|^{2} \\
\leq & \sum_{j=1}^{n} \mathrm{E}\left|\frac{1}{2 \pi \mathbf{i}} \oint_{|\zeta-z|=v / 2} \frac{\left[\log \left(1+\epsilon_{j}(\zeta) \bar{\beta}_{j}(\zeta)\right)-\epsilon_{j}(\zeta) \bar{\beta}_{j}(\zeta)\right]}{(z-\zeta)^{2}} d \zeta\right|^{2} \\
\leq & \frac{1}{2 \pi v^{4}} \sum_{j=1}^{n} \oint_{|\zeta-z|=v / 2} \mathrm{E}\left|\epsilon_{j}(\zeta) \bar{\beta}_{j}(\zeta)\right|^{4} d \zeta
\end{aligned}
$$




$$
\begin{aligned}
\leq & \frac{K}{2 \pi n^{4} v^{4}} \sum_{j=1}^{n} \oint_{|\zeta-z|=v / 2}\left\{\mathrm{E}\left[\operatorname{tr} \mathbf{T}_{n} \mathbf{D}_{j}^{-1}(\zeta) \mathbf{T}_{n} \mathbf{D}_{j}^{-1}(z)(\bar{\zeta})\right]^{2}\right. \\
& \left.+\sum_{i=1}^{k} \mathrm{E}\left|x_{i j}\right|^{8} \mathrm{E}\left|\mathbf{q}_{i}^{T} \mathbf{D}_{j}^{-1}(z) \mathbf{q}_{i}\right|^{4} d \zeta\right\} \\
\leq & K n^{-1}+K \eta_{n}^{4} \rightarrow 0 .
\end{aligned}
$$

Therefore, we need only to derive the finite dimensional limiting distribution of

$$
\frac{d}{d z} \sum_{j=1}^{n}\left(\mathrm{E}_{j}-\mathrm{E}_{j-1}\right) \epsilon_{j}(z) \bar{\beta}_{j}(z)=\frac{d}{d z} \sum_{j=1}^{n} \mathrm{E}_{j} \epsilon_{j}(z) \bar{\beta}_{j}(z) .
$$

Similar to the last three lines of the proof of (15.23), one can show that

$$
\begin{aligned}
& \sum_{j=1}^{n} \mathrm{E}\left|\left(\mathrm{E}_{j}-\mathrm{E}_{j-1}\right) \frac{d}{d z} \epsilon_{j}(z) \bar{\beta}_{j}(z)\right|^{2} I\left(\left|\left(\mathrm{E}_{j}-\mathrm{E}_{j-1}\right) \frac{d}{d z} \epsilon_{j}(z) \bar{\beta}_{j}(z)\right| \geq \epsilon\right) \\
\leq & \frac{1}{\epsilon^{2}} \sum_{j=1}^{n} \mathrm{E}\left|\mathrm{E}_{j} \frac{d}{d z} \epsilon_{j}(z) \bar{\beta}_{j}(z)\right|^{4} \rightarrow 0 .
\end{aligned}
$$

Thus, the martingale difference sequence $\left\{\left(\mathrm{E}_{j}-\mathrm{E}_{j-1}\right) \frac{d}{d z} \epsilon_{j}(z) \bar{\beta}_{j}(z)\right\}$ satisfies the Lyapunov condition. Applying Lemma A.4 the random vector $\left(M_{n}^{1}\left(z_{1}\right), \cdots, M_{n}^{1}\left(z_{r}\right)\right)$ will tend to an $r$-dimensional Gaussian vector $\left(M\left(z_{1}\right), \cdots, M\left(z_{r}\right)\right)$ whose covariance function is given by

$$
\operatorname{Cov}\left(M\left(z_{1}\right), M\left(z_{2}\right)\right)=\lim _{n \rightarrow \infty} \sum_{j=1}^{n} \mathrm{E}_{j-1}\left(\mathrm{E}_{j} \frac{\partial}{\partial z_{1}} \epsilon_{j}\left(z_{1}\right) \bar{\beta}_{j}\left(z_{1}\right) \cdot \mathrm{E}_{j} \frac{\partial}{\partial z_{2}} \epsilon_{j}\left(z_{2}\right) \bar{\beta}_{j}\left(z_{2}\right)\right) .
$$

Consider the sum $\Gamma_{n}\left(z_{1}, z_{2}\right)=\sum_{j=1}^{n} \mathrm{E}_{j-1}\left[\mathrm{E}_{j}\left(\bar{\beta}_{j}\left(z_{1}\right) \epsilon_{j}\left(z_{1}\right)\right) \mathrm{E}_{j}\left(\bar{\beta}_{j}\left(z_{2}\right) \epsilon_{j}\left(z_{2}\right)\right)\right]$. Using the same approach of Bai and Silverstein (2004), we may replace $\bar{\beta}_{j}(z)$ by $b_{n}(z)$. Therefore, by (1.5) of Bai and Silverstein (2004), we have

$$
\begin{aligned}
\Gamma_{n}\left(z_{1}, z_{2}\right)=\sum_{j=1}^{n} b_{n}\left(z_{1}\right) b_{n}\left(z_{2}\right) \mathrm{E}_{j-1}[ & \left.\mathrm{E}_{j}\left(\epsilon_{j}\left(z_{1}\right)\right) \mathrm{E}_{j}\left(\epsilon_{j}\left(z_{2}\right)\right)\right] \\
=\frac{1}{n^{2}} \sum_{j=1}^{n} b_{n}\left(z_{1}\right) b_{n}\left(z_{2}\right) \operatorname{tr}[ & \mathrm{E}_{j}\left(\mathbf{T}_{n} \mathbf{D}_{j}^{-1}\left(z_{1}\right)\right) \mathrm{E}_{j}\left(\mathbf{T}_{n} \mathbf{D}_{j}^{-1}\left(z_{2}\right)\right) \\
& +\alpha_{x} \operatorname{tr} \mathrm{E}_{j} \overline{\mathbf{Q}} \mathbf{Q}^{*} \mathbf{D}_{j}^{-1}\left(z_{1}\right) \mathbf{Q} \mathbf{Q}^{T} \mathrm{E}_{j}\left(\mathbf{D}_{j}^{T}\right)^{-1}\left(z_{2}\right) \\
& \left.+\beta_{x} \sum_{i=1}^{k} \mathbf{q}_{i}^{*} \mathrm{E}_{j} \mathbf{D}_{j}^{-1}\left(z_{1}\right) \mathbf{q}_{i} \mathbf{q}_{i}^{*} \mathrm{E}_{j} \mathbf{D}_{j}^{-1}\left(z_{2}\right) \mathbf{q}_{i}\right] \\
=\frac{1}{n^{2}} \sum_{j=1}^{n} b_{n}\left(z_{1}\right) b_{n}\left(z_{2}\right) \operatorname{tr} & {\left[\mathrm{E}_{j}\left(\mathbf{T}_{n} \mathbf{D}_{j}^{-1}\left(z_{1}\right)\right) \mathrm{E}_{j}\left(\mathbf{T}_{n} \mathbf{D}_{j}^{-1}\left(z_{2}\right)\right)\right.}
\end{aligned}
$$




$$
\left.\begin{array}{l}
+\alpha_{x} \operatorname{tr} \mathbf{T}_{n} \mathrm{E}_{j} \mathbf{D}_{j}^{-1}\left(z_{1}\right) \mathbf{T}_{n} \mathrm{E}_{j}\left(\mathbf{D}_{j}^{T}\right)^{-1}\left(z_{2}\right) \\
+\beta_{x} \sum_{i=1}^{k} \mathbf{q}_{i}^{*} \mathrm{E}_{j} \mathbf{D}_{j}^{-1}\left(z_{1}\right) \mathbf{q}_{i} \mathbf{q}_{i}^{*} \mathrm{E}_{j} \mathbf{D}_{j}^{-1}\left(z_{2}\right) \mathbf{q}_{i}
\end{array}\right]
$$

where $\alpha_{x}=\left|E x_{11}^{2}\right|^{2}$ and $\beta_{x}=E\left|x_{11}^{4}\right|-\alpha_{x}-2$. Here, the third equality holds if either $\alpha_{x}=0$ or $\mathbf{Q}$ is real which implies that $\mathbf{T}_{n}=\mathbf{Q Q}^{T}=\overline{\mathbf{Q}} \mathbf{Q}^{*}$.

Now we use the new method to derive the limit of the first term which is different from but easier than that used in Bai and Silverstein (2004). Let $v_{0}$ be a lower bound on $\Im\left(z_{i}\right)$. Define $\breve{\mathbf{r}}_{j}$ as an i.i.d. copy of $\mathbf{r}_{j}, j=1, \cdots, n$ and define $\breve{\mathbf{D}}_{j}(z)$ similar as $\mathbf{D}_{j}(z)$ by using $\mathbf{r}_{1}, \cdots, \mathbf{r}_{j-1}, \breve{\mathbf{r}}_{j+1} \cdots, \breve{\mathbf{r}}_{n}$. Then we have

$$
\operatorname{tr}\left[\mathrm{E}_{j} \mathbf{T}_{n} \mathbf{D}_{j}^{-1}\left(z_{1}\right) \mathrm{E}_{j}\left(\mathbf{T}_{n} \mathbf{D}_{j}^{-1}\left(z_{2}\right)\right)\right]=\operatorname{tr} \mathrm{E}_{j}\left[\mathbf{T}_{n} \mathbf{D}_{j}^{-1}\left(z_{1}\right) \mathbf{T}_{n} \breve{\mathbf{D}}_{j}^{-1}\left(z_{2}\right)\right]
$$

Similar to (5.15), one can prove that

$$
n^{-1} \mathrm{E}_{j}\left[z_{1} \operatorname{tr} \mathbf{T}_{n} \mathbf{D}_{j}^{-1}\left(z_{1}\right)-z_{2} \operatorname{tr} \mathbf{T}_{n} \breve{\mathbf{D}}_{j}^{-1}\left(z_{2}\right)\right] \rightarrow z_{1}\left[b^{-1}\left(z_{1}\right)-1\right]-z_{2}\left[b^{-1}\left(z_{2}\right)-1\right], \text { a.s. }
$$

where $b(z)=\lim _{n \rightarrow \infty} b_{n}(z)=-z \underline{m}(z)$. On the other hand,

$$
\begin{aligned}
& \mathrm{E}_{j} n^{-1}\left[z_{1} \operatorname{tr} \mathbf{T}_{n} \mathbf{D}_{j}^{-1}\left(z_{1}\right)-z_{2} \operatorname{tr} \mathbf{T}_{n} \breve{\mathbf{D}}_{j}^{-1}\left(z_{2}\right)\right] \\
= & n^{-1} \mathrm{E}_{j} \operatorname{tr} \mathbf{T}_{n} \mathbf{D}_{j}^{-1}\left(z_{1}\right)\left[\left(z_{1}-z_{2}\right) \sum_{i=1}^{j-1} \mathbf{r}_{i} \mathbf{r}_{i}^{*}+\sum_{i=j+1}^{n}\left(z_{1} \mathbf{r}_{i} \mathbf{r}_{i}^{*}-z_{2} \breve{\mathbf{r}}_{i} \breve{\mathbf{r}}_{i}^{*}\right)\right] \breve{\mathbf{D}}_{j}^{-1}\left(z_{2}\right) \\
= & n^{-1} \sum_{i=1}^{j-1}\left(z_{1}-z_{2}\right) \mathrm{E}_{j} \mathbf{r}_{i}^{*} \breve{\mathbf{D}}_{j i}^{-1}\left(z_{2}\right) \mathbf{T}_{n} \mathbf{D}_{j i}^{-1}\left(z_{1}\right) \mathbf{r}_{i} \beta_{j i}\left(z_{1}\right) \breve{\beta}_{j i}\left(z_{2}\right) \\
& +n^{-1} \sum_{i=j+1}^{n} \mathrm{E}_{j}\left[z_{1} \breve{\beta}_{j i}\left(z_{2}\right) \mathbf{r}_{i}^{*} \breve{\mathbf{D}}_{j i}^{-1}\left(z_{2}\right) \mathbf{T}_{n} \mathbf{D}_{j}^{-1}\left(z_{1}\right) \mathbf{r}_{i}-z_{2} \beta_{j i}\left(z_{1}\right) \breve{\mathbf{r}}_{i}^{*} \breve{\mathbf{D}}_{j}^{-1}\left(z_{2}\right) \mathbf{T}_{n} \mathbf{D}_{j i}^{-1}\left(z_{1}\right) \breve{\mathbf{r}}_{i}\right] \\
= & n^{-2} \sum_{i=1}^{j-1}\left(z_{1}-z_{2}\right) \mathrm{E}_{j} \operatorname{tr} \mathbf{T}_{n} \breve{\mathbf{D}}_{j i}^{-1}\left(z_{2}\right) \mathbf{T}_{n} \mathbf{D}_{j i}^{-1}\left(z_{1}\right) b\left(z_{1}\right) b\left(z_{2}\right) \\
& +n^{-2} \sum_{i=j+1}^{n} \mathrm{E}_{j}\left[z_{1} b\left(z_{2}\right) \operatorname{tr} \mathbf{T}_{n} \breve{\mathbf{D}}_{j i}^{-1}\left(z_{2}\right) \mathbf{T}_{n} \mathbf{D}_{j}^{-1}\left(z_{1}\right)-z_{2} b\left(z_{1}\right) \operatorname{tr} \mathbf{T}_{n} \breve{\mathbf{D}}_{j}^{-1}\left(z_{2}\right) \mathbf{T}_{n} \mathbf{D}_{j i}^{-1}\left(z_{1}\right)\right]+o_{a . s .}(1) \\
& \left(\operatorname{by~replacing} \mathbf{D}_{j i}^{-1}=\mathbf{D}_{j}^{-1}+\mathbf{D}_{j i}^{-1} \mathbf{r}_{i} \mathbf{r}_{i}^{*} \mathbf{D}_{j i}^{-1} \beta_{j i}\right) \\
= & {\left[\frac{j-1}{n}\left(z_{1}-z_{2}\right) b\left(z_{1}\right) b\left(z_{2}\right)+\frac{n-j}{n}\left(z_{1} b\left(z_{2}\right)-z_{2} b\left(z_{1}\right)\right)\right] \mathrm{E}_{j} n^{-1} \operatorname{tr}_{n} \breve{\mathbf{D}}_{j}^{-1}\left(z_{2}\right) \mathbf{T}_{n} \mathbf{D}_{j}^{-1}\left(z_{1}\right)+o_{a . s .}(1) . }
\end{aligned}
$$

Comparing the two estimates, we obtain

$$
\mathrm{E}_{j} \frac{1}{n} \operatorname{tr} \mathbf{T}_{n} \breve{\mathbf{D}}_{j}^{-1}\left(z_{2}\right) \mathbf{T}_{n} \mathbf{D}_{j}^{-1}\left(z_{1}\right)=\frac{z_{1}\left(b^{-1}\left(z_{1}\right)-1\right)-z_{2}\left(b^{-1}\left(z_{2}\right)-1\right)+o_{a . s .}(1)}{\frac{j-1}{n}\left(z_{1}-z_{2}\right) b\left(z_{1}\right) b\left(z_{2}\right)+\frac{n-j}{n}\left(z_{1} b\left(z_{2}\right)-z_{2} b\left(z_{1}\right)\right)}
$$


Consequently, we obtain

$$
\begin{aligned}
& \frac{1}{n^{2}} \sum_{j=1}^{n} b_{n}\left(z_{1}\right) b_{n}\left(z_{2}\right) \operatorname{tr} \mathrm{E}_{j}\left[\mathbf{T}_{n} \mathbf{D}_{j}^{-1}\left(z_{1}\right) \mathrm{E}_{j}\left(\mathbf{T}_{n} \mathbf{D}_{j}^{-1}\left(z_{2}\right)\right)\right] \\
\rightarrow & a\left(z_{1}, z_{2}\right) \int_{0}^{1} \frac{1}{1-t a\left(z_{1}, z_{2}\right)} d t=-\log \left(1-a\left(z_{1}, z_{2}\right)\right)=\int_{0}^{a\left(z_{1}, z_{2}\right)} \frac{1}{1-z} d z,
\end{aligned}
$$

where

$$
\begin{aligned}
a\left(z_{1}, z_{2}\right) & =\frac{b\left(z_{1}\right) b\left(z_{2}\right)\left[z_{1}\left(b^{-1}\left(z_{1}\right)-1\right)-z_{2}\left(b^{-1}\left(z_{2}\right)-1\right)\right]}{z_{1} b\left(z_{2}\right)-z_{2} b\left(z_{1}\right)} \\
& =1+\frac{b\left(z_{1}\right) b\left(z_{2}\right)\left(z_{2}-z_{1}\right)}{z_{1} b\left(z_{2}\right)-z_{2} b\left(z_{1}\right)}=1+\frac{\underline{m}\left(z_{1}\right) \underline{m}\left(z_{2}\right)\left(z_{1}-z_{2}\right)}{\underline{m}\left(z_{2}\right)-\underline{m}\left(z_{1}\right)} .
\end{aligned}
$$

Thus, we have

$$
\begin{aligned}
& \frac{\partial^{2}}{\partial z_{2} \partial z_{1}} \int_{0}^{a\left(z_{1}, z_{2}\right)} \frac{1}{1-z} d z \\
= & \frac{\partial}{\partial z_{2}}\left(\frac{\frac{\partial}{\partial z_{1}} a\left(z_{1}, z_{2}\right)}{1-a\left(z_{1}, z_{2}\right)}\right) \\
= & \frac{\partial}{\partial z_{2}}\left[\frac{\left(\underline{m}\left(z_{2}\right)-\underline{m}\left(z_{1}\right)\right)\left(\frac{\partial \underline{m}\left(z_{1}\right)}{\partial z_{1}} \underline{m}\left(z_{2}\right)\left(z_{1}-z_{2}\right)+\underline{m}\left(z_{1}\right) \underline{m}\left(z_{2}\right)\right)+\underline{m}\left(z_{1}\right) \underline{m}\left(z_{2}\right)\left(z_{1}-z_{2}\right) \frac{m\left(z_{1}\right)}{\partial z_{1}}}{\left(\underline{m}\left(z_{2}\right)-\underline{m}\left(z_{1}\right)\right)^{2}}\right] \\
= & -\frac{\partial}{\partial z_{2}}\left(\frac{\underline{m}\left(z_{2}\right)-\underline{m}\left(z_{1}\right)}{\left.\underline{m}\left(z_{1}\right) / \partial z_{1}\right)}+\frac{1}{z_{1}}\left(z_{1}\right)\right. \\
= & \frac{\left(\partial \underline{m}\left(z_{1}\right) / \partial z_{1}\right)\left(\partial \underline{m}\left(z_{2}\right) / \partial z_{2}\right)}{\left(\underline{m}\left(z_{2}\right)-\underline{m}\left(z_{1}\right)\right)^{2}}-\frac{\left(\partial \underline{m}\left(z_{2}\right)-\underline{m}\left(z_{1}\right) / \partial z_{1}\right)}{\left(z_{1}-z_{2}\right)^{2}} .
\end{aligned}
$$

Next, we compute the limit of the second term of (5.25). In this step, we need the assumption that the matrix $\mathbf{Q}$ is real. Similarly, we consider

$$
\frac{1}{n} \mathrm{E}_{j}\left[z_{1} \operatorname{tr} \mathbf{T}_{n} \mathbf{D}_{j}^{-1}\left(z_{1}\right)-z_{2} \operatorname{tr} \mathbf{T}_{n}\left(\breve{\mathbf{D}}_{j}^{T}\right)^{-1}\left(z_{2}\right)\right] \rightarrow z_{1}\left(b^{-1}\left(z_{1}\right)-1\right)-z_{2}\left(b^{-1}\left(z_{2}\right)-1\right), \text { a.s. }
$$

On the other hand,

$$
\begin{aligned}
& n^{-1} \mathrm{E}_{j}\left[z_{1} \operatorname{tr} \mathbf{T} \mathbf{D}_{j}^{-1}\left(z_{1}\right)-z_{2} \operatorname{tr} \mathbf{T}\left(\breve{\mathbf{D}}_{j}^{T}\right)^{-1}\left(z_{2}\right)\right] \\
= & n^{-1} \mathrm{E}_{j} \operatorname{tr} \mathbf{T} \mathbf{D}_{j}^{-1}\left(z_{1}\right)\left[\sum_{i=1}^{j-1}\left(z_{1} \mathbf{r}_{i} \mathbf{r}_{i}^{*}-z_{2} \overline{\mathbf{r}}_{i} \mathbf{r}_{i}^{T}\right)+\sum_{i=j+1}^{n}\left(z_{1} \mathbf{r}_{i} \mathbf{r}_{i}^{*}-z_{2} \breve{\mathbf{r}}_{i} \breve{\mathbf{r}}_{i}^{T}\right)\right]\left(\breve{\mathbf{D}}_{j}^{T}\right)^{-1}\left(z_{2}\right)
\end{aligned}
$$




$$
\begin{aligned}
= & n^{-1} \sum_{i=1}^{j-1} \mathrm{E}_{j}\left[z_{1} \breve{\beta}_{j i}\left(z_{2}\right) \mathbf{r}_{i}^{*}\left(\breve{\mathbf{D}}_{j i}^{T}\right)^{-1}\left(z_{2}\right) \mathbf{T}\left[\mathbf{D}_{j i}^{-1}\left(z_{1}\right)-\mathbf{D}_{j i}^{-1}\left(z_{1}\right) \mathbf{r}_{i} \mathbf{r}_{i}^{*} \mathbf{D}_{j i}^{-1}\left(z_{1}\right) \beta_{j i}\left(z_{1}\right)\right] \mathbf{r}_{i}\right. \\
& \left.\left.-z_{2} \mathbf{r}_{i}^{T}\left(\left(\breve{\mathbf{D}}_{j i}^{T}\right)^{-1}\left(z_{2}\right)-\breve{\beta}_{j i}\left(z_{2}\right)\left(\breve{\mathbf{D}}_{j i}^{T}\right)^{-1}\left(z_{2}\right) \overline{\mathbf{r}}_{i} \mathbf{r}_{i}^{T}\left(\breve{\mathbf{D}}_{j i}^{T}\right)^{-1}\left(z_{2}\right)\right) \mathbf{T D}_{j i}^{-1}\left(z_{1}\right) \beta_{j i}\left(z_{1}\right)\right] \overline{\mathbf{r}}_{i}\right] \\
& +n^{-1} \sum_{i=j+1}^{n} \mathrm{E}_{j}\left[z_{1} \breve{\beta}_{j i}\left(z_{2}\right) \mathbf{r}_{i}^{*}\left(\breve{\mathbf{D}}_{j i}^{T}\right)^{-1}\left(z_{2}\right) \mathbf{T D}_{j}^{-1}\left(z_{1}\right) \mathbf{r}_{i}-z_{2} \breve{\mathbf{r}}_{i}^{T} \breve{\mathbf{D}}_{j}^{-1}\left(z_{2}\right) \mathbf{T D}_{j i}^{-1}\left(z_{1}\right) \overline{\mathbf{r}}_{i} \beta_{j i}\left(z_{1}\right)\right] \\
= & \left\{(j-1) n^{-1} \alpha_{x}\left[-\left(z_{1} b\left(z_{2}\right)-z_{2} b\left(z_{1}\right)\right)+b\left(z_{1}\right) b\left(z_{2}\right)\left(z_{1}-z_{2}\right)\right]+\left(z_{1} b\left(z_{2}\right)-z_{2} b\left(z_{1}\right)\right)\right\} \\
& \mathrm{E}_{j} n^{-1} \operatorname{tr}_{\mathbf{T}} \mathbf{D D}_{j}^{-1}\left(z_{1}\right) \mathbf{T}\left(\breve{\mathbf{D}}_{j}^{T}\right)^{-1}\left(z_{2}\right)+o_{a . s .}(1) .
\end{aligned}
$$

Comparing the two estimates, we obtain

$$
\begin{aligned}
& \mathrm{E}_{j} n^{-1} \operatorname{tr} \mathbf{T D}_{j}^{-1}\left(z_{2}\right) \mathbf{T D}_{j}^{-1}\left(z_{1}\right) \\
= & \frac{z_{1}\left(b^{-1}\left(z_{1}\right)-1\right)-\left(z_{2} b^{-1}\left(z_{2}\right)-1\right)+o_{a . s .}(1)}{(j-1) n^{-1} \alpha_{x}\left[-\left(z_{1} b\left(z_{2}\right)-z_{2} b\left(z_{1}\right)\right)+b\left(z_{1}\right) b\left(z_{2}\right)\left(z_{1}-z_{2}\right)\right]+\left[z_{1} b\left(z_{2}\right)-z_{2} b\left(z_{1}\right)\right]} .
\end{aligned}
$$

Consequently, we obtain

$$
\begin{aligned}
& n^{-2} \sum_{j=1}^{n} \alpha_{x} b_{n}\left(z_{1}\right) b_{n}\left(z_{2}\right) \operatorname{tr} \mathrm{E}_{j} \mathbf{T D}_{j}^{-1}\left(z_{1}\right) \mathrm{E}_{j}\left(\mathbf{T}\left(\mathbf{D}_{j}^{T}\right)^{-1}\left(z_{2}\right)\right) \\
\rightarrow & \widetilde{a}\left(z_{1}, z_{2}\right) \int_{0}^{1} \frac{1}{1-t \widetilde{a}\left(z_{1}, z_{2}\right)} d t=-\log \left(1-\widetilde{a}\left(z_{1}, z_{2}\right)\right)=\int_{0}^{\widetilde{a}\left(z_{1}, z_{2}\right)} \frac{1}{1-z} d z
\end{aligned}
$$

where

$$
\begin{aligned}
\widetilde{a}\left(z_{1}, z_{2}\right) & =\frac{\alpha_{x} b\left(z_{1}\right) b\left(z_{2}\right)\left(z_{1}\left(b^{-1}\left(z_{1}\right)-1\right)-z_{2}\left(b^{-1}\left(z_{2}\right)-1\right)\right)}{z_{1} b\left(z_{2}\right)-z_{2} b\left(z_{1}\right)} \\
& =\alpha_{x}\left(1+\frac{b\left(z_{1}\right) b\left(z_{2}\right)\left(z_{2}-z_{1}\right)}{z_{1} b\left(z_{2}\right)-z_{2} b\left(z_{1}\right)}\right)=\alpha_{x}\left(1+\frac{\underline{m}\left(z_{1}\right) \underline{m}\left(z_{2}\right)\left(z_{1}-z_{2}\right)}{\underline{m}\left(z_{2}\right)-\underline{m}\left(z_{1}\right)}\right) .
\end{aligned}
$$

Last, we will compute the limit of the third term of (5.25). By (9.9.12) of Bai and Silverstein (2010), we have

$$
\begin{aligned}
& n^{-2} \sum_{j=1}^{n} \beta_{x} \sum_{i=1}^{k} \mathbf{e}_{i}^{T} \mathbf{Q}^{*} \mathbf{D}_{j}^{-1}\left(z_{1}\right) \mathbf{Q} \mathbf{e}_{i} \mathbf{e}_{i}^{T} \mathbf{Q}^{*} \mathbf{D}_{j}^{-1}\left(z_{2}\right) \mathbf{Q} \mathbf{e}_{i} \\
= & \frac{1}{n^{2} z_{1} z_{2}} \sum_{j=1}^{n} \beta_{x} \sum_{i=1}^{k} \mathbf{e}_{i}^{T} \mathbf{Q}^{*}\left(\underline{m}\left(z_{1}\right) \mathbf{T}_{n}+\mathbf{I}_{p}\right)^{-1} \mathbf{Q} \mathbf{e}_{i} \mathbf{e}_{i}^{T} \mathbf{Q}^{*}\left(\underline{m}\left(z_{2}\right) \mathbf{T}_{n}+\mathbf{I}_{p}\right)^{-1} \mathbf{Q} \mathbf{e}_{i}+o_{p}(1)
\end{aligned}
$$


If $\beta_{x} \neq 0$, then by assumption, the matrix $\mathbf{Q}^{*} \mathbf{Q}$ is diagonal. Using the identity $\mathbf{Q}^{*}\left[\underline{m}(z) \mathbf{T}_{n}+\right.$ $\left.\mathbf{I}_{p}\right]^{-1} \mathbf{Q}=\underline{m}^{-1}(z)\left\{\mathbf{I}_{k}-\left[\underline{m}(z) \mathbf{Q}^{*} \mathbf{Q}+\mathbf{I}_{k}\right]^{-1}\right\}$, we see that the matrix is diagonal. We have

$$
\begin{aligned}
& \frac{1}{n^{2} z_{1} z_{2}} \sum_{j=1}^{n} \beta_{x} \sum_{i=1}^{k} \mathbf{e}_{i}^{T} \mathbf{Q}^{*}\left[\underline{m}\left(z_{1}\right) \mathbf{T}_{n}+\mathbf{I}_{p}\right]^{-1} \mathbf{Q} \mathbf{e}_{i} \mathbf{e}_{i}^{T} \mathbf{Q}^{*}\left[\underline{m}\left(z_{2}\right) \mathbf{T}_{n}+\mathbf{I}_{p}\right]^{-1} \mathbf{Q} \mathbf{e}_{i} \\
= & \frac{1}{n^{2} z_{1} z_{2}} \sum_{j=1}^{n} \beta_{x} \operatorname{tr}\left\{\mathbf{Q}^{*}\left[\underline{m}\left(z_{1}\right) \mathbf{T}_{n}+\mathbf{I}_{p}\right]^{-1} \mathbf{Q} \mathbf{Q}^{*}\left[\underline{m}\left(z_{2}\right) \mathbf{T}_{n}+\mathbf{I}_{p}\right]^{-1} \mathbf{Q}\right\} \\
= & \frac{y \beta_{x}}{z_{1} z_{2}} \int \frac{t^{2}}{\left[1+t \underline{m}\left(z_{1}\right)\right]\left[1+t \underline{m}\left(z_{2}\right)\right]} d H(t)+o(1) .
\end{aligned}
$$

Then the third term of $\operatorname{Cov}\left(M\left(z_{1}\right), M\left(z_{2}\right)\right)$ is

$$
\begin{aligned}
& \frac{\partial^{2}}{\partial z_{1} \partial z_{2}}\left\{\frac{y \beta_{x} b\left(z_{1}\right) b\left(z_{2}\right)}{z_{1} z_{2}} \int \frac{t^{2}}{\left[1+t \underline{m}\left(z_{1}\right)\right]\left[1+t \underline{m}\left(z_{2}\right)\right]} d H(t)\right\} \\
= & \frac{\partial^{2}}{\partial z_{1} \partial z_{2}}\left\{y \beta_{x} \int \frac{t^{2} \underline{m}\left(z_{1}\right) \underline{m}\left(z_{2}\right)}{\left[1+t \underline{m}\left(z_{1}\right)\right]\left[1+t \underline{m}\left(z_{2}\right)\right]} d H(t)\right\} \\
= & y \beta_{x} \frac{\partial \underline{m}\left(z_{1}\right)}{\partial z_{1}} \frac{\partial \underline{m}\left(z_{2}\right)}{\partial z_{2}} \int \frac{t^{2}}{\left[1+t \underline{m}\left(z_{1}\right)\right]^{2}\left[1+t \underline{m}\left(z_{2}\right)\right]^{2}} d H(t) .
\end{aligned}
$$

Tightness of $M_{n}^{1}(z)$. As done in Bai and Silverstein (2004), the proof of tightness of $M_{n}^{1}$ relies on the proof of

$$
\begin{aligned}
& \sup _{n, z_{1}, z_{2} \in \mathcal{C}} \mathrm{E}\left|\frac{\widehat{M}_{n}^{1}\left(z_{1}\right)-\widehat{M}_{n}\left(z_{2}\right)-\mathrm{E}\left(\widehat{M}_{n}\left(z_{1}\right)-\widehat{M}_{n}\left(z_{2}\right)\right)}{z_{1}-z_{2}}\right|^{2} \\
& =\sup _{\substack{n, z_{1}, z_{2} \in \mathcal{C} \\
\left|\Im\left(z_{i}\right)\right|>n}} \mathrm{E}\left|\operatorname{tr} \mathbf{D}^{-1}\left(z_{1}\right) \mathbf{D}^{-1}\left(z_{2}\right)-\mathrm{Etr}_{\epsilon_{n}} \mathbf{D}^{-1}\left(z_{1}\right) \mathbf{D}^{-1}\left(z_{2}\right)\right|^{2} \\
& :=\sup _{\substack{n, z_{1}, z_{2} \in \mathcal{C} \\
\left|\Im\left(z_{i}\right)\right|>n}} J_{n}\left(z_{1}, z_{2}\right)<\infty
\end{aligned}
$$

where by the formula (5.22), we have

$$
\begin{aligned}
& J_{n}\left(z_{1}, z_{2}\right) \\
= & \mathrm{E}\left|\sum_{j=1}^{n}\left(\mathrm{E}_{j}-\mathrm{E}_{j-1}\right) \operatorname{tr} \mathbf{D}^{-1}\left(z_{1}\right) \mathbf{D}^{-1}\left(z_{2}\right)\right|^{2} \\
= & \sum_{j=1}^{n} \mathrm{E}\left|\left(\mathrm{E}_{j}-\mathrm{E}_{j-1}\right) \mathbf{r}_{j}^{*} \mathbf{D}_{j}^{-1}\left(z_{1}\right) \mathbf{D}_{j}^{-1}\left(z_{2}\right) \mathbf{D}_{j}^{-1}\left(z_{1}\right) \mathbf{r}_{j} \beta_{j}\left(z_{1}\right)\right|^{2} \\
& +\sum_{j=1}^{n} \mathrm{E}\left|\left(\mathrm{E}_{j}-\mathrm{E}_{j-1}\right) \mathbf{r}_{j}^{*} \mathbf{D}_{j}^{-1}\left(z_{2}\right) \mathbf{D}_{j}^{-1}\left(z_{1}\right) \mathbf{D}_{j}^{-1}\left(z_{2}\right) \mathbf{r}_{j} \beta_{j}\left(z_{2}\right)\right|^{2}
\end{aligned}
$$




$$
\begin{aligned}
& +\sum_{j=1}^{n} \mathrm{E}\left|\left(\mathrm{E}_{j}-\mathrm{E}_{j-1}\right) \mathbf{r}_{j}^{*} \mathbf{D}_{j}^{-1}\left(z_{1}\right) \mathbf{D}_{j}^{-1}\left(z_{2}\right) \mathbf{r}_{j} \mathbf{r}_{j}^{*} \mathbf{D}_{j}^{-1}\left(z_{2}\right) \mathbf{D}_{j}^{-1}\left(z_{1}\right) \mathbf{r}_{j} \beta_{j}\left(z_{1}\right) \beta_{j}\left(z_{2}\right)\right|^{2} \\
= & \sum_{j=1}^{n} \mathrm{E}\left|\left(\mathrm{E}_{j}-\mathrm{E}_{j-1}\right) \mathbf{r}_{j}^{*} \mathbf{D}_{j}^{-1}\left(z_{1}\right) \mathbf{D}_{j}^{-1}\left(z_{2}\right) \mathbf{D}_{j}^{-1}\left(z_{1}\right) \mathbf{r}_{j} b_{j}\left(z_{1}\right)\right|^{2} \\
& +\sum_{j=1}^{n} \mathrm{E}\left|\left(\mathrm{E}_{j}-\mathrm{E}_{j-1}\right) \mathbf{r}_{j}^{*} \mathbf{D}_{j}^{-1}\left(z_{2}\right) \mathbf{D}_{j}^{-1}\left(z_{1}\right) \mathbf{D}_{j}^{-1}\left(z_{2}\right) \mathbf{r}_{j} b_{j}\left(z_{2}\right)\right|^{2} \\
& +\sum_{j=1}^{n} \mathrm{E}\left|\left(\mathrm{E}_{j}-\mathrm{E}_{j-1}\right) \mathbf{r}_{j}^{*} \mathbf{D}_{j}^{-1}\left(z_{1}\right) \mathbf{D}_{j}^{-1}\left(z_{2}\right) \mathbf{r}_{j} \mathbf{r}_{j}^{*} \mathbf{D}_{j}^{-1}\left(z_{2}\right) \mathbf{D}_{j}^{-1}\left(z_{1}\right) \mathbf{r}_{j} b_{j}\left(z_{1}\right) b_{j}\left(z_{2}\right)\right|^{2}+O(1) \\
= & O(1)
\end{aligned}
$$

Convergence of $M_{n}^{2}(z)$. In order to simplify the exposition, we let $\mathcal{C}_{1}=\mathcal{C}_{u}$ or $\mathcal{C}_{u} \cup \mathcal{C}_{l}$ if $x_{l}<0$, and $\mathcal{C}_{2}=\mathcal{C}_{r}$ or $\mathcal{C}_{r} \cup \mathcal{C}_{l}$ if $x_{l}>0$. We begin with proving

$$
\sup _{z \in \mathcal{C}_{n}}\left|\operatorname{E} \underline{m}_{n}(z)-\underline{m}(z)\right| \rightarrow 0 \quad \text { as } n \rightarrow \infty \text {. }
$$

Since $F \underline{\mathbf{B}}_{n} \downarrow F^{y, H}$ almost surely, we get from d.c.t. $\mathrm{E} F \underline{\mathbf{B}}_{n} \downarrow F^{y, H}$. It is easy to verify that $\mathrm{E} F \underline{B}_{n}$ is a proper c.d.f. Since, as $z$ ranges in $\mathcal{C}_{1}$, the functions $(\lambda-z)^{-1}$ in $\lambda \in[0, \infty)$ form a bounded, equicontinuous family, it follows [see, e.g. Billingsley (1968), Problem 8, p. 17] that

$$
\sup _{z \in \mathcal{C}_{1}}\left|\underline{\operatorname{E}}_{n}(z)-\underline{m}(z)\right| \rightarrow 0 .
$$

For $z \in \mathcal{C}_{2}$, we write $\left(\eta_{l}, \eta_{r}\right.$ defined as in previous section)

$\mathrm{E}_{n}(z)-m(z)=\int \frac{1}{\lambda-z} I_{\left[\eta_{l}, \eta_{r}\right]}(\lambda) d\left(\mathrm{EF}^{\underline{\mathbf{B}}_{n}}(\lambda)-F^{c, H}(\lambda)\right)+\mathrm{E} \int \frac{1}{\lambda-z} I_{\left[\eta_{l}, \eta_{r}\right]^{c}}(\lambda) d F^{\underline{\mathbf{B}}_{n}}(\lambda)$.

As above, the first term converges uniformly to zero. With $\ell \geq 2$, we get

$$
\begin{aligned}
& \sup _{z \in \mathcal{C}_{2}}\left|\mathrm{E} \int \frac{1}{\lambda-z} I_{\left[\eta_{l}, \eta_{r}\right]^{c}}(\lambda) d F^{\mathbf{B}_{n}}(\lambda)\right| \\
& \leq\left(\epsilon_{n} / n\right)^{-1} P\left(\left\|\mathbf{B}_{n}\right\| \geq \eta_{r} \text { or } \lambda_{\min }^{\mathbf{B}_{n}} \leq \eta_{l}\right) \leq K n \epsilon_{n}^{-1} n^{-\ell} \rightarrow 0 .
\end{aligned}
$$

From the fact that $F^{y_{n}, H_{n}} \downarrow F^{y, H}$ [see Bai and Silverstein (1998), below (3.10)] along with the fact that $\mathcal{C}$ lies outside the support of $F^{c, H}$, it is straightforward to verify that

$$
\sup _{z \in \mathcal{C}}\left|\underline{m}_{n}^{0}(z)-\underline{m}(z)\right| \rightarrow 0 \quad \text { as } n \rightarrow \infty
$$

where $m_{n}^{0}(z)$ is the Stieltjes transform of $F^{y_{n}, H_{n}}$. We now show that

$$
\sup _{n, z \in \mathcal{C}_{n}}\left\|\left(\underline{\mathrm{E}}_{n}(z) \mathbf{T}_{n}+\mathbf{I}_{p}\right)^{-1}\right\|<\infty
$$


From Lemma 2.11 of Bai and Silverstein (1998), $\left\|\left(\mathrm{E} \underline{m}_{n}(z) \mathbf{T}_{n}+\mathbf{I}_{p}\right)^{-1}\right\|$ is bounded by $\max \left(2,4 v_{0}^{-1}\right)$ on $\mathcal{C}_{u}$. On $\mathcal{C}_{l}$, the boundedness of $\left\|\left(\underline{E}_{n}(z) \mathbf{T}_{n}+\mathbf{I}_{p}\right)^{-1}\right\|$ follows from the fact that

$$
\left|\mathrm{E} \underline{m}_{n}(z) \lambda_{j}+1\right| \geq \Re\left(\mathrm{E} \underline{m}_{n}(z) \lambda_{j}+1\right) \geq P\left(\lambda_{\min }\left(\mathbf{B}_{n}\right)>\eta_{l}\right)-\epsilon_{n}^{-1} P\left(\lambda_{\min }\left(\mathbf{B}_{n}\right)<\eta_{l}\right) \rightarrow 1,
$$

where $\lambda_{j}$ is an arbitrary eigenvalue of $\mathbf{T}_{n}$ and $\|\mathbf{Q}\| \leq 1$. Now let us consider the bound on $\mathcal{C}_{r}$. By (1.1) of Bai and Silverstein (1998), there exists a support point $t_{0}$ of $H$ such that $1+t_{0} \underline{m}(z) \neq 0$ on $\mathcal{C}_{r}$. Since $\underline{m}(z)$ is analytic on $\mathcal{C}_{r}$, there exist positive constants $\delta_{1}$ and $\mu_{0}$ such that

$$
\inf _{z \in \mathcal{C}_{r}}\left|1+t_{0} \underline{m}(z)\right|>\delta_{1}, \quad \text { and } \quad \sup _{z \in \mathcal{C}_{r}}|\underline{m}(z)|<\mu_{0} .
$$

By (5.27) and $H_{n} \rightarrow H$, for all large $n$, there exists an integer $j \leq n$ such that $\left|\lambda_{j}-t_{0}\right|<$ $\delta_{1} / 4 \mu_{0}$ and $\sup _{z \in \mathcal{C}_{r}}\left|E \underline{m}_{n}(z)-\underline{m}(z)\right|<\delta_{1} / 4$. Then, we have

$$
\inf _{z \in \mathcal{C}_{r}}\left|1+\lambda_{j} \underline{\mathrm{E}}_{n}(z)\right|>\delta_{1} / 2,
$$

which completes the proof of (5.29).

Next we show the existence of $\xi \in(0,1)$ such that for all $n$ large

$$
\sup _{z \in \mathcal{C}_{n}}\left|y_{n} \underline{\mathrm{E}}_{n}(z)^{2} \int \frac{t^{2}}{\left(1+t \mathrm{E} \underline{m}_{n}(z)\right)^{2}} d H_{n}(t)\right|<\xi \text {. }
$$

From the identity (1.1) of Bai and Silverstein (1998)

$$
\underline{m}(z)=\frac{1}{-z+y \int \frac{t}{1+\underline{t} \underline{m}(z)} d H(t)}
$$

valid for $z=x+i v$ outside the support of $F^{y, H}$, we find

$$
\Im \underline{m}(z)=\frac{v+\Im \underline{m}(z) y \int \frac{t^{2}}{|1+t \underline{m}(z)|^{2}} d H(t)}{\left|-z+y \int \frac{t}{1+t \underline{m}(z)} d H(t)\right|^{2}} .
$$

Therefore

$$
\begin{aligned}
\left|y \underline{m}(z)^{2} \int \frac{t^{2}}{(1+t \underline{m}(z))^{2}} d H(t)\right| & \leq \frac{y \int \frac{t^{2}}{|1+\underline{\underline{m}}(z)|^{2}} d H(t)}{\left|-z+y \int \frac{t}{1+t \underline{m}(z)} d H(t)\right|^{2}} \\
& =\frac{\Im \underline{m}(z) y \int \frac{t^{2}}{|1+\underline{t}(z)|^{2}} d H(t)}{v+\Im \underline{m}(z) y \int \frac{t^{2}}{1+\left.t \underline{t}(z)\right|^{2}} d H(t)}
\end{aligned}
$$




$$
=\frac{y \int \frac{1}{|x-z|^{2}} d F^{y, H}(x) \int \frac{t^{2}}{|1+t \underline{m}(z)|^{2}} d H(t)}{1+y \int \frac{1}{|x-z|^{2}} d F^{y, H}(x) \int \frac{t^{2}}{|1+t \underline{m}(z)|^{2}} d H(t)}<1,5
$$

for all $z \in \mathcal{C}$. By continuity, we have $\xi_{1}<1$ such that

$$
\sup _{z \in \mathcal{C}}\left|y \underline{m}(z)^{2} \int \frac{t^{2}}{(1+t \underline{m}(z))^{2}} d H(t)\right|<\xi_{1} .
$$

Therefore, using (5.27), (5.30) follows. We proceed with some improved bounds on quantities appearing earlier. Recall the functions $\beta_{j}(z)$ and $\gamma_{j}(z)$ defined in (5.5) . For $p \geq 4$, we have

$$
\mathrm{E}\left|\gamma_{1}(z)\right|^{p} \leq K n^{-2}
$$

Let $\mathbf{M}$ be nonrandom $p \times p$. Then

$$
\begin{aligned}
& \mathrm{E}\left|\operatorname{tr} \mathbf{D}^{-1}(z) \mathbf{M}-\mathrm{Etr} \mathbf{D}^{-1}(z) \mathbf{M}\right|^{2} \\
= & \mathrm{E}\left|\sum_{j=1}^{n} \mathrm{E}_{j} \operatorname{tr} \mathbf{D}^{-1}(z) \mathbf{M}-\mathrm{E}_{j-1} \operatorname{tr} \mathbf{D}^{-1}(z) \mathbf{M}\right|^{2} \\
= & \mathrm{E}\left|\sum_{j=1}^{n}\left(\mathrm{E}_{j}-\mathrm{E}_{j-1}\right) \operatorname{tr}\left(\mathbf{D}^{-1}(z)-\mathbf{D}_{j}^{-1}(z)\right) \mathbf{M}\right|^{2} \\
= & \sum_{j=1}^{n} \mathrm{E}\left|\left(\mathrm{E}_{j}-\mathrm{E}_{j-1}\right) \beta_{j}(z) \mathbf{r}_{j}^{*} \mathbf{D}_{j}^{-1}(z) \mathbf{M D}_{j}^{-1}(z) \mathbf{r}_{j}\right|^{2} \\
= & \left|b_{n}(z)\right|^{2} \sum_{j=1}^{n} \mathrm{E}\left|\left(\mathrm{E}_{j}-\mathrm{E}_{j-1}\right)\left(1-\beta_{j}(z) \gamma_{j}(z)\right) \mathbf{r}_{j}^{*} \mathbf{D}_{j}^{-1}(z) \mathbf{M D}_{j}^{-1}(z) \mathbf{r}_{j}\right|^{2} \\
= & \left|b_{n}(z)\right|^{2} \sum_{j=1}^{n} \mathrm{E} \mid \mathrm{E}_{j}\left(\mathbf{r}_{j}^{*} \mathbf{D}_{j}^{-1}(z) \mathbf{M D}_{j}^{-1}(z) \mathbf{r}_{j}-n^{-1} \operatorname{tr}_{j}^{-1}(z) \mathbf{M D}_{j}^{-1}(z) \mathbf{T}_{n}\right. \\
& -\left.\left(\mathrm{E}_{j}-\mathrm{E}_{j-1}\right) \beta_{j}(z) \gamma_{j}(z) \mathbf{r}_{j}^{*} \mathbf{D}_{j}^{-1}(z) \mathbf{M D}_{j}^{-1}(z) \mathbf{r}_{j}\right|^{2} \\
\leq & 2\left|b_{n}(z)\right|^{2} n \mathrm{E}\left|\mathbf{r}_{j}^{*} \mathbf{D}_{j}^{-1}(z) \mathbf{M D}_{j}^{-1}(z) \mathbf{r}_{j}-n^{-1} \operatorname{tr}_{j}^{-1}(z) \mathbf{M D}_{j}^{-1}(z) \mathbf{T}_{n}\right|^{2} \\
& +4\left|b_{n}(z)\right|^{2} n \mathrm{E}\left|\beta_{j}(z) \gamma_{j}(z) \mathbf{r}_{j}^{*} \mathbf{D}_{j}^{-1}(z) \mathbf{M D}_{j}^{-1}(z) \mathbf{r}_{j}\right|^{2} \\
\leq & 2\left|b_{n}(z)\right|^{2} n \mathrm{E}\left|\mathbf{r}_{j}^{*} \mathbf{D}_{j}^{-1}(z) \mathbf{M D}_{j}^{-1}(z) \mathbf{r}_{j}-n^{-1} \operatorname{trD}_{j}^{-1}(z) \mathbf{M D}_{j}^{-1}(z) \mathbf{T}_{n}\right|^{2} \\
& +4\left|b_{n}(z)\right|^{2} n\left(\mathrm{E}\left|\gamma_{j}(z)\right|^{4}\right)^{1 / 2}\left(\left.\mathrm{E} \beta_{j}(z)\right|^{8}\right)^{1 / 4}\left(\left.\mathrm{E} \mathbf{r}_{j}^{*} \mathbf{D}_{j}^{-1}(z) \mathbf{M D}_{j}^{-1}(z) \mathbf{r}_{j}\right|^{8}\right)^{1 / 4} .
\end{aligned}
$$

Using (9.9.3) of Bai and Silverstein (2010), (5.33) and the boundedness of $b_{n}(z)$ we get

$$
\mathrm{E}\left|\operatorname{tr} \mathbf{D}^{-1}(z) \mathbf{M}-\mathrm{Etr}^{-1}(z) \mathbf{M}\right|^{2} \leq K\|\mathbf{M}\|^{2} .
$$

The same argument holds for $\mathbf{D}_{1}^{-1}(z)$ so we also have

$$
\mathrm{E}\left|\operatorname{tr} \mathbf{D}_{1}^{-1}(z) \mathbf{M}-\mathrm{Etr}_{1}^{-1}(z) \mathbf{M}\right|^{2} \leq K\|\mathbf{M}\|^{2} .
$$


Our next task is to investigate the limiting behavior of

$$
\begin{aligned}
& n\left(y_{n} \int \frac{d H_{n}(t)}{1+t \underline{\mathrm{E}} \underline{m}_{n}(z)}+z y_{n} \operatorname{E} m_{n}(z)\right) \\
= & n \operatorname{E} \beta_{1}(z)\left[\mathbf{r}_{1}^{*} \mathbf{D}_{1}^{-1}(z)\left(\mathrm{E} \underline{m}_{n}(z) \mathbf{T}_{n}+\mathbf{I}_{p}\right)^{-1} \mathbf{r}_{1}-n^{-1} \operatorname{Etr}\left(\operatorname{E} \underline{m}_{n}(z) \mathbf{T}_{n}+\mathbf{I}_{p}\right)^{-1} \mathbf{T}_{n} \mathbf{D}^{-1}(z)\right],
\end{aligned}
$$

for $z \in \mathcal{C}_{n}$ [see (5.2) in Bai and Silverstein (1998)]. Throughout the following, all bounds, including $O(\cdot)$ and $o(\cdot)$ expressions, and convergence statements hold uniformly for $z \in \mathcal{C}_{n}$. We have

$$
\begin{aligned}
& \operatorname{Etr}\left(\operatorname{E} \underline{m}_{n}(z) \mathbf{T}_{n}+\mathbf{I}_{p}\right)^{-1} \mathbf{T}_{n} \mathbf{D}_{1}^{-1}(z)-\operatorname{Etr}\left(\operatorname{E} \underline{m}_{n}(z) \mathbf{T}_{n}+\mathbf{I}_{p}\right)^{-1} \mathbf{T}_{n} \mathbf{D}^{-1}(z) \\
= & \operatorname{E} \beta_{1}(z) \operatorname{tr}\left(\operatorname{E} \underline{m}_{n}(z) \mathbf{T}_{n}+\mathbf{I}_{p}\right)^{-1} \mathbf{T}_{n} \mathbf{D}_{1}^{-1}(z) \mathbf{r}_{1} \mathbf{r}_{1}^{*} \mathbf{D}_{1}^{-1}(z) \\
= & b_{n}(z) \mathrm{E}\left(1-\beta_{1}(z) \gamma_{1}(z)\right) \mathbf{r}_{1}^{*} \mathbf{D}_{1}^{-1}(z)\left(\operatorname{E} \underline{m}_{n}(z) \mathbf{T}_{n}+\mathbf{I}_{p}\right)^{-1} \mathbf{T}_{n} \mathbf{D}_{1}^{-1}(z) \mathbf{r}_{1}
\end{aligned}
$$

From (5.29) we get

$$
\begin{aligned}
& \left|\mathrm{E} \beta_{1}(z) \gamma_{1}(z) \mathbf{r}_{1}^{*} \mathbf{D}_{1}^{-1}(z)\left(\mathrm{E}_{n}(z) \mathbf{T}_{n}+\mathbf{I}_{p}\right)^{-1} \mathbf{T}_{n} \mathbf{D}_{1}^{-1}(z) \mathbf{r}_{1}\right| \\
\leq & \left.\left.\left(\mathrm{E}\left|\gamma_{1}(z)\right|^{2}\right)^{1 / 2}\right)\left(\mathrm{E}\left|\beta_{1}(z)\right|^{4}\right)^{1 / 4}\right)\left(\mathrm{E}\left|\mathbf{r}_{1}^{*} \mathbf{D}_{1}^{-1}(z)\left(\mathrm{E} \underline{m}_{n}(z) \mathbf{T}_{n}+\mathbf{I}_{p}\right)^{-1} \mathbf{T}_{n} \mathbf{D}_{1}^{-1}(z) \mathbf{r}_{1}\right|^{4}\right)^{1 / 4} \\
\leq & K n^{-1 / 2}
\end{aligned}
$$

Therefore

$$
\left|(\underline{5.36})-n^{-1} b_{n}(z) \operatorname{Etr}^{-1}(z)\left(\underline{\operatorname{Em}}_{n}(z) \mathbf{T}_{n}+\mathbf{I}_{p}\right)^{-1} \mathbf{T}_{n} \mathbf{D}_{1}^{-1}(z) \mathbf{T}_{n}\right| \leq K n^{-1 / 2} .
$$

Since $\beta_{1}(z)=b_{n}(z)-b_{n}^{2}(z) \gamma_{1}(z)+\beta_{1}(z) b_{n}^{2}(z) \gamma_{1}^{2}(z)$, we have

$$
\begin{aligned}
& n \mathrm{E} \beta_{1}(z) \mathbf{r}_{1}^{*} \mathbf{D}_{1}^{-1}(z)\left(\mathrm{E} \underline{m}_{n}(z) \mathbf{T}_{n}+\mathbf{I}_{p}\right)^{-1} \mathbf{r}_{1}-\mathrm{E} \beta_{1}(z) \operatorname{Etr}\left(\operatorname{E} \underline{m}_{n}(z) \mathbf{T}_{n}+\mathbf{I}_{p}\right)^{-1} \mathbf{T}_{n} \mathbf{D}_{1}^{-1}(z) \\
= & -b_{n}^{2}(z) n \mathrm{E} \gamma_{1}(z) \mathbf{r}_{1}^{*} \mathbf{D}_{1}^{-1}(z)\left(\mathrm{E} \underline{m}_{n}(z) \mathbf{T}_{n}+\mathbf{I}_{p}\right)^{-1} \mathbf{r}_{1} \\
& +b_{n}^{2}(z)\left(n \mathrm{E} \beta_{1}(z) \gamma_{1}^{2}(z) \mathbf{r}_{1}^{*} \mathbf{D}_{1}^{-1}(z)\left(\mathrm{E} \underline{m}_{n}(z) \mathbf{T}_{n}+\mathbf{I}_{p}\right)^{-1} \mathbf{r}_{1}\right. \\
& \left.-\left(\mathrm{E} \beta_{1}(z) \gamma_{1}^{2}(z)\right) \operatorname{Etr}\left(\mathrm{E} \underline{m}_{n}(z) \mathbf{T}_{n}+\mathbf{I}_{p}\right)^{-1} \mathbf{T}_{n} \mathbf{D}_{1}^{-1}(z)\right) \\
= & -b_{n}^{2}(z) n \mathrm{E} \gamma_{1}(z) \mathbf{r}_{1}^{*} \mathbf{D}_{1}^{-1}(z)\left(\mathrm{E} \underline{m}_{n}(z) \mathbf{T}_{n}+\mathbf{I}_{p}\right)^{-1} \mathbf{r}_{1} \\
& +b_{n}^{2}(z)\left(\mathrm { E } \left[n \beta_{1}(z) \gamma_{1}^{2}(z) \mathbf{r}_{1}^{*} \mathbf{D}_{1}^{-1}(z)\left(\mathrm{E} \underline{m}_{n}(z) \mathbf{T}_{n}+\mathbf{I}_{p}\right)^{-1} \mathbf{r}_{1}\right.\right. \\
& \left.\left.-\beta_{1}(z) \gamma_{1}^{2}(z) \operatorname{tr} \mathbf{D}_{1}^{-1}(z)\left(\underline{\mathrm{E}}_{n}(z) \mathbf{T}_{n}+\mathbf{I}_{p}\right)^{-1} \mathbf{T}_{n}\right]\right) \\
& +b_{n}^{2}(z) \operatorname{Cov}\left(\beta_{1}(z) \gamma_{1}^{2}(z), \operatorname{tr} \mathbf{D}_{1}^{-1}(z)\left(\mathrm{E} \underline{m}_{n}(z) \mathbf{T}_{n}+\mathbf{I}_{p}\right)^{-1} \mathbf{T}_{n}\right)
\end{aligned}
$$

$(\operatorname{Cov}(X, Y)=\mathrm{E} X Y-\mathrm{E} X \mathrm{E} Y)$. Using (5.29), (15.33), (5.35) and Lemma A.2, we have

$$
\left|\mathrm{E}\left[n \beta_{1}(z) \gamma_{1}^{2}(z) \mathbf{r}_{1}^{*} \mathbf{D}_{1}^{-1}(z)\left(\mathrm{E}_{n}(z) \mathbf{T}_{n}+\mathbf{I}_{p}\right)^{-1} \mathbf{r}_{1}-\beta_{1}(z) \gamma_{1}^{2}(z) \operatorname{tr} \mathbf{D}_{1}^{-1}(z)\left(\mathrm{E} \underline{m}_{n}(z) \mathbf{T}_{n}+\mathbf{I}_{p}\right)^{-1} \mathbf{T}_{p}\right]\right|
$$




$$
\begin{aligned}
\leq & n\left(\mathrm{E}\left|\beta_{1}(z)\right|^{4}\right)^{1 / 4}\left(\mathrm{E}\left|\gamma_{1}(z)\right|^{4}\right)^{1 / 2}\left(\mathrm{E} \mid \mathbf{r}_{1}^{*} \mathbf{D}_{1}^{-1}(z)\left(\mathrm{E} \underline{m}_{n}(z) \mathbf{T}_{n}+\mathbf{I}_{p}\right)^{-1} \mathbf{r}_{1}\right. \\
& \left.-\left.n^{-1} \operatorname{tr}_{1}^{-1}\left(\underline{E}_{n}(z) \mathbf{T}_{n}+\mathbf{I}_{p}\right)^{-1} \mathbf{T}_{n}\right|^{4}\right)^{1 / 4} \\
\leq & K n^{-1 / 2}
\end{aligned}
$$

and

$$
\begin{aligned}
& \left|\operatorname{Cov}\left(\beta_{1}(z) \gamma_{1}^{2}(z), \operatorname{tr} \mathbf{D}_{1}^{-1}(z)\left(\underline{E}_{n}(z) \mathbf{T}_{n}+\mathbf{I}_{p}\right)^{-1} \mathbf{T}_{p}\right)\right| \\
& \leq\left(\mathrm{E}\left|\beta_{1}(z)\right|^{6}\right)^{1 / 6}\left(\mathrm{E}\left|\gamma_{1}(z)\right|^{6}\right)^{1 / 3}\left(\mathrm{E} \mid \operatorname{tr}_{1}^{-1}(z)\left(\mathrm{E} \underline{m}_{n}(z) \mathbf{T}_{n}+\mathbf{I}_{p}\right)^{-1} \mathbf{T}_{n}\right. \\
& \left.-\left.\operatorname{Etr}_{1}^{-1}(z)\left(\underline{\operatorname{Em}}_{n}(z) \mathbf{T}_{n}+\mathbf{I}_{p}\right)^{-1} \mathbf{T}_{n}\right|^{2}\right)^{1 / 2} \\
& \leq K n^{-2 / 3} \text {. }
\end{aligned}
$$

Since $\beta_{1}(z)=b_{n}(z)-b_{n}(z) \beta_{1}(z) \gamma_{1}(z)$, then we have $\mathrm{E} \beta_{1}(z)=b_{n}(z)+O\left(n^{-1 / 2}\right)$. Write

$$
\begin{aligned}
& \operatorname{En} \gamma_{1}(z) \mathbf{r}_{1}^{*} \mathbf{D}_{1}^{-1}(z)\left(\underline{E}_{n}(z) \mathbf{T}_{n}+\mathbf{I}_{p}\right)^{-1} \mathbf{r}_{1} \\
= & n \mathrm{E}\left[( \mathbf { r } _ { 1 } ^ { * } \mathbf { D } _ { 1 } ^ { - 1 } ( z ) \mathbf { r } _ { 1 } - n ^ { - 1 } \operatorname { t r } _ { 1 } ^ { - 1 } ( z ) \mathbf { T } _ { n } ) \left(\mathbf{r}_{1}^{*} \mathbf{D}_{1}^{-1}(z)\left(\mathrm{E} \underline{m}_{n}(z) \mathbf{T}_{n}+\mathbf{I}_{p}\right)^{-1} \mathbf{r}_{1}\right.\right. \\
& \left.\left.-n^{-1} \operatorname{tr}_{1}^{-1}(z)\left(\mathrm{E} \underline{m}_{n}(z) \mathbf{T}_{n}+\mathbf{I}_{p}\right)^{-1} \mathbf{T}_{n}\right)\right] \\
& +n^{-1} \operatorname{Cov}\left(\operatorname{tr} \mathbf{D}_{1}^{-1}(z) \mathbf{T}_{n}, \operatorname{tr}_{1}^{-1}(z)\left(\operatorname{E} \underline{m}_{n}(z) \mathbf{T}_{n}+\mathbf{I}_{p}\right)^{-1} \mathbf{T}_{n}\right) .
\end{aligned}
$$

From (5.35) we see that the second term above is $O\left(n^{-1}\right)$. Therefore, we arrive at

$$
\begin{aligned}
& n\left(y_{n} \int \frac{d H_{n}(t)}{1+t \mathrm{E} \underline{m}_{n}}+z y_{n} \operatorname{Em}_{n}(z)\right) \\
& =b_{n}^{2}(z) n^{-1} \operatorname{Etr}_{1}^{-1}(z)\left(\underline{E}_{n}(z) \mathbf{T}_{n}+\mathbf{I}_{p}\right)^{-1} \mathbf{T}_{n} \mathbf{D}_{1}^{-1}(z) \mathbf{T}_{n} \\
& -b_{n}^{2}(z) n \mathrm{E}\left[( \mathbf { r } _ { 1 } ^ { * } \mathbf { D } _ { 1 } ^ { - 1 } ( z ) \mathbf { r } _ { 1 } - n ^ { - 1 } \operatorname { t r } \mathbf { D } _ { 1 } ^ { - 1 } ( z ) \mathbf { T } _ { n } ) \left(\mathbf{r}_{1}^{*} \mathbf{D}_{1}^{-1}(z)\left(\mathrm{E}_{n}(z) \mathbf{T}_{n}+\mathbf{I}_{p}\right)^{-1} \mathbf{r}_{1}\right.\right. \\
& \left.\left.-n^{-1} \operatorname{tr} \mathbf{D}_{1}^{-1}(z)\left(\mathrm{E}_{n}(z) \mathbf{T}_{n}+\mathbf{I}_{p}\right)^{-1} \mathbf{T}_{n}\right)\right]+o(1) \\
& =-\frac{b_{n}^{2}(z)}{n} \beta_{x} \sum_{i=1}^{m} \mathbf{q}_{i}^{*} \mathbf{D}_{1}^{-1}(z) \mathbf{q}_{i} \cdot \mathbf{q}_{i}^{*} \mathbf{D}_{1}^{-1}(z)\left(\underline{\mathrm{E}}_{n}(z) \mathbf{T}_{n}+\mathbf{I}\right)^{-1} \mathbf{q}_{i} \\
& -\frac{b_{n}^{2}(z)}{n} \alpha_{x} \operatorname{tr} \mathbf{T}_{n}\left(\mathbf{D}_{1}^{T}(z)\right)^{-1} \mathbf{T}_{n} \mathbf{D}_{1}^{-1}(z)\left(\mathrm{E}_{n}(z) \mathbf{T}_{n}+\mathbf{I}\right)^{-1} \text {. }
\end{aligned}
$$

Let $A_{n}(z)=y_{n} \int \frac{d H_{n}(t)}{1+t \mathrm{E} \underline{m}_{n}(z)}+z y_{n} \operatorname{E} m_{n}(z)$. Using the identity $\underline{\operatorname{E}}_{n}(z)=-\frac{\left(1-y_{n}\right)}{z}+y_{n} \operatorname{E} m_{n}(z)$, we have

$$
\begin{aligned}
A_{n}(z)= & y_{n} \int \frac{d H_{n}(t)}{1+t \mathrm{E} \underline{m}_{n}(z)}-y_{n}+z \mathrm{E} \underline{m}_{n}(z)+1 \\
& =-\operatorname{E} \underline{m}_{n}(z)\left(-z-\frac{1}{\operatorname{E} \underline{m}_{n}(z)}+y_{n} \int \frac{t d H_{n}(t)}{1+t \operatorname{E} \underline{m}_{n}(z)}\right) .
\end{aligned}
$$

It follows that

$$
\mathrm{E} \underline{m}_{n}(z)=\frac{1}{-z+y_{n} \int \frac{t d H_{n}(t)}{1+t \underline{\underline{m}}_{n}(z)}+A_{n} / \mathrm{E} \underline{m}_{n}(z)}
$$


From this, together with the analogous identity [below (4.4)] we get

$$
\mathrm{E} \underline{\underline{m}}_{n}(z)-\underline{m}_{n}^{0}(z)=-\frac{\underline{m}_{n}^{0}(z) A_{n}}{1-y_{n} \mathrm{E} \underline{m}_{n}(z) \underline{m}_{n}^{0}(z) \int \frac{t^{2} d H_{n}(t)}{\left(1+t \underline{\mathrm{E}} \underline{m}_{n}(z)\right)\left(1+\underline{m}_{n}^{0}(z)\right)} .}
$$

We see from (5.30) and the corresponding bound involving $\underline{m}_{n}^{0}(z)$, that the denominator of (5.37) is bounded away from zero. First we have

$$
\begin{aligned}
& \alpha_{x} \frac{z^{2} \underline{m}^{2}(z)}{n^{2}} \sum_{j=1}^{n} \operatorname{Etr}\left[\mathbf{D}_{1}^{-1}(z)\left\{\underline{m}(z) \mathbf{T}_{n}+\mathbf{I}_{p}\right\}^{-1} \mathbf{T}_{n}\left(\mathbf{D}_{1}^{T}(z)\right)^{-1} \mathbf{T}_{n}\right] \\
= & \alpha_{x} \frac{\underline{m}^{2}(z)}{n} \operatorname{Etr}\left(\underline{m}(z) \mathbf{T}_{n}+\mathbf{I}_{p}\right)^{-3} \mathbf{T}_{n}^{2} \\
& +\alpha_{x} \frac{\underline{z}^{2} \underline{m}^{4}(z)}{n^{2}} \sum_{i \neq j} \operatorname{Etr}\left\{\left(\underline{m}(z) \mathbf{T}_{n}+\mathbf{I}_{p}\right)^{-1} \mathbf{T}_{n} \underline{m}(z) \mathbf{T}_{n}+\mathbf{I}_{p}\right)^{-1}\left(\mathbf{r}_{i} \mathbf{r}_{i}^{*}-\frac{1}{n} \mathbf{T}_{n}\right) \mathbf{A}_{i j}^{-1}(z) \\
& \left.\cdot\left(\underline{m}(z) \mathbf{T}_{n}+\mathbf{I}_{p}\right)^{-1} \mathbf{T}_{n}\left(\mathbf{A}_{i j}^{T}\right)^{-1}(z)\left(\overline{\mathbf{r}}_{i} \mathbf{r}_{i}^{T}-\frac{1}{n} \mathbf{T}_{n}\right)\right\}+o(1) \\
= & \alpha_{x} \frac{\underline{m}^{2}(z)}{n} \operatorname{tr}\left(\underline{m}(z) \mathbf{T}_{n}+\mathbf{I}_{p}\right)^{-3} \mathbf{T}_{n}^{2} \\
& +\frac{\alpha_{x}^{2} z^{2} \underline{m}^{4}(z)}{n^{3}} \sum_{j=1}^{n}\left\{\operatorname{tr}\left(\underline{m}(z) \mathbf{T}_{n}+\mathbf{I}_{p}\right)^{-1} \mathbf{T}_{n}\right\}^{2} \cdot \operatorname{tr} \mathbf{D}_{1}^{-1}(z)\left(\underline{m}(z) \mathbf{T}_{n}+\mathbf{I}_{p}\right)^{-1} \mathbf{T}_{n}\left(\mathbf{A}_{j}^{T}(z)\right)^{-1} \mathbf{T}_{n}+o(1) .
\end{aligned}
$$

Then we have

$$
\begin{aligned}
& \alpha_{x} \frac{z^{2} \underline{m}^{2}(z)}{n^{2}} \sum_{j=1}^{n} \operatorname{Etr}\left[\mathbf{D}_{1}^{-1}(z)\left\{\underline{m}(z) \mathbf{T}_{n}+\mathbf{I}_{p}\right\}^{-1} \mathbf{T}_{n}\left(\mathbf{D}_{1}^{T}(z)\right)^{-1} \mathbf{T}_{n}\right] \\
& =\frac{\alpha_{x} \frac{\underline{m}^{2}(z)}{n} \operatorname{tr}\left\{\left(\underline{m}(z) \mathbf{T}_{n}+\mathbf{I}_{p}\right)^{-3} \mathbf{T}_{n}^{2}\right\}}{1-\frac{\alpha_{x} \underline{m}^{2}(z)}{n} \operatorname{tr}\left\{\left(\underline{m}(z) \mathbf{T}_{n}+\mathbf{I}_{p}\right)^{-1} \mathbf{T}_{n}\right\}^{2}}+o(1) \\
& =\frac{\alpha_{x} y \int \frac{\underline{m}^{2}(z) t^{2} d H(t)}{(1+t \underline{m}(z))^{3}}}{1-\alpha_{x} y \int \frac{\underline{m}^{2}(z) t^{2} d H(t)}{(1+\underline{m}(z))^{2}}}+o(1) .
\end{aligned}
$$

Thus we obtain

$$
\begin{aligned}
& \frac{\alpha_{x} \frac{z^{2} \underline{m}^{2}(z)}{n^{2}} \sum_{j=1}^{n} \operatorname{Etr}\left[\mathbf{D}_{1}^{-1}(z)\left\{\underline{m}(z) \mathbf{T}_{n}+\mathbf{I}_{p}\right\}^{-1} \mathbf{T}_{n}\left(\mathbf{D}_{1}^{T}(z)\right)^{-1} \mathbf{T}_{n}\right]}{1-y \int \frac{\underline{m}^{2}(z) t^{2} d H(t)}{(1+t \underline{m}(z))^{2}}} \\
= & \frac{\alpha_{x} y \int \frac{\underline{m}^{2}(z) t^{2} d H(t)}{(1+\underline{\underline{m}}(z))^{3}}}{\left\{1-y \int \frac{\underline{m}^{2}(z) t^{2} d H(t)}{(1+t \underline{m}(z))^{2}}\right\}\left\{1-\alpha_{x} y \int \frac{\underline{m}^{2}(z) t^{2} d H(t)}{(1+t \underline{m}(z))^{2}}\right\}}+o(1) .
\end{aligned}
$$


Moreover, if $\beta_{x}=0$ or $\mathbf{Q}^{*} \mathbf{Q}$ is diagonal, then we have

$$
\begin{aligned}
& \frac{\beta_{x} y_{n} z^{2} \underline{m}^{3}(z) \frac{1}{p n} \sum_{j=1}^{n} \sum_{i=1}^{m} \operatorname{E} \mathbf{q}_{i}^{*} \mathbf{D}_{1}^{-1}(z)\left\{\underline{m}(z) \mathbf{T}_{n}+\mathbf{I}_{p}\right\}^{-1} \mathbf{q}_{i} \cdot \mathbf{q}_{i}^{*} \mathbf{D}_{1}^{-1}(z) \mathbf{q}_{i}}{1-y \int \frac{\underline{m}^{2}(z) t^{2} d H(t)}{(1+t \underline{m}(z))^{2}}} \\
= & \beta_{x} \frac{y \int \frac{\underline{m}^{3}(z) t^{2}}{\left(\underline{\underline{m}(z) t+1)^{3}} d H(t)\right.}}{1-y \int \frac{\underline{m}^{2}(z) t^{2} d H(t)}{(1+\underline{m}(z))^{2}}}+o(1)
\end{aligned}
$$

where

$$
\begin{aligned}
& \mathbf{q}_{i}^{*} \mathbf{D}_{1}^{-1}(z)\left\{\underline{m}(z) \mathbf{T}_{n}+\mathbf{I}_{p}\right\}^{-1} \mathbf{q}_{i} \cdot \mathbf{q}_{i}^{*} \mathbf{D}_{1}^{-1}(z) \mathbf{q}_{i} \\
= & \mathbf{e}_{i}^{T} \mathbf{Q}^{*} \mathbf{D}_{1}^{-1}(z)\left\{\underline{m}(z) \mathbf{T}_{n}+\mathbf{I}_{p}\right\}^{-1} \mathbf{Q} \mathbf{e}_{i} \mathbf{e}_{i}^{T} \mathbf{Q}^{*} \mathbf{D}_{1}^{-1}(z) \mathbf{Q} \mathbf{e}_{i} \\
= & z^{-2} \mathbf{e}_{i}^{T} \mathbf{Q}^{*}\left\{\underline{m}(z) \mathbf{T}_{n}+\mathbf{I}_{p}\right\}^{-2} \mathbf{Q} \mathbf{e}_{i} \mathbf{e}_{i}^{T} \mathbf{Q}^{*}\left\{\underline{m}(z) \mathbf{T}_{n}+\mathbf{I}_{p}\right\}^{-1} \mathbf{Q} \mathbf{e}_{i} \\
= & z^{-2} \mathbf{e}_{i}^{T} \mathbf{Q}^{*}\left\{\underline{m}(z) \mathbf{T}_{n}+\mathbf{I}_{p}\right\}^{-1} \mathbf{Q} \mathbf{Q}^{*}\left(\mathbf{Q} \mathbf{Q}^{*}\right)^{-1}\left\{\underline{m}(z) \mathbf{T}_{n}+\mathbf{I}_{p}\right\}^{-1} \mathbf{Q} \mathbf{e}_{i} \mathbf{e}_{i}^{T} \mathbf{Q}^{*}\left\{\underline{m}(z) \mathbf{T}_{n}+\mathbf{I}_{p}\right\}^{-1} \mathbf{Q} \mathbf{e}_{i} \\
= & z^{-2} \mathbf{e}_{i}^{T} \mathbf{Q}^{*}\left\{\underline{m}(z) \mathbf{T}_{n}+\mathbf{I}_{p}\right\}^{-1} \mathbf{Q} \mathbf{Q}^{*}\left(\mathbf{Q} \mathbf{Q}^{*}\right)^{-1 / 2}\left\{\underline{m}(z) \mathbf{T}_{n}+\mathbf{I}_{p}\right\}^{-1}\left(\mathbf{Q Q}^{*}\right)^{-1 / 2} \mathbf{Q} \mathbf{e}_{i} \\
& \cdot \mathbf{e}_{i}^{T} \mathbf{Q}^{*}\left\{\underline{m}(z) \mathbf{T}_{n}+\mathbf{I}_{p}\right\}^{-1} \mathbf{Q} \mathbf{e}_{i},
\end{aligned}
$$

and

$$
\begin{gathered}
\mathbf{Q}^{*}\left(\mathbf{Q} \mathbf{Q}^{*}\right)^{-1 / 2}\left(\underline{m}(z) \mathbf{Q} \mathbf{Q}^{*}+\mathbf{I}_{p}\right)^{-1}\left(\mathbf{Q} \mathbf{Q}^{*}\right)^{-1 / 2} \mathbf{Q}=\mathbf{I}_{k}-\left[\mathbf{Q}^{*} \mathbf{Q}+\underline{m}^{-1}(z) \mathbf{I}_{k}\right]^{-1} \mathbf{Q}^{*} \mathbf{Q} \\
\mathbf{Q}^{*}\left(\underline{m}(z) \mathbf{Q} \mathbf{Q}^{*}+\mathbf{I}_{p}\right)^{-1} \mathbf{Q}=\underline{m}^{-1}(z)\left\{\mathbf{I}_{k}-\left[\mathbf{I}_{k}+\underline{m}(z) \mathbf{Q}^{*} \mathbf{Q}\right]^{-1}\right\} .
\end{gathered}
$$

That is,

$$
\begin{aligned}
& \mathrm{E}_{n}(z)-\underline{m}_{n}^{0}(z) \\
= & -\frac{\underline{m}_{n}^{0}(z) A_{n}(z)}{1-c_{n} \mathrm{E} \underline{m}_{n}(z) \underline{m}_{n}^{0}(z) \int \frac{t^{2} d H_{n}(t)}{\left(1+t \underline{\underline{m}}_{n}(z)\right)\left(1+t \underline{\underline{m}}_{n}^{0}(z)\right)}} \\
= & \frac{\alpha_{x} y \int \frac{\underline{m}^{3}(z) t^{2} d H(t)}{(1+t \underline{\underline{m}}(z))^{3}}}{\left(1-y \int \frac{\underline{m}^{2}(z) t^{2} d H(t)}{(1+t \underline{m}(z))^{2}}\right)\left(1-\alpha_{x} y \int \frac{\underline{m}^{2}(z) t^{2} d H(t)}{(1+t \underline{m}(z))^{2}}\right)}+\beta_{x} \frac{y \int \frac{\underline{m}^{3}(z) t^{2}}{\left(\underline{\underline{m}(z) t+1)^{3}} d H(t)\right.}}{1-y \int \frac{\underline{m}^{2}(z) t^{2} d H(t)}{(1+t \underline{m}(z))^{2}}} .
\end{aligned}
$$

\subsection{Proof of Corollary 3.3}

Let $a(y)=(1-\sqrt{y})^{2}$ and $b(y)=(1+\sqrt{y})^{2}$, then for $\ell \in\{1, \ldots, L\}$, we have

$$
E X_{f_{\ell}}=\frac{[a(y)]^{\ell}+[b(y)]^{\ell}}{4}-\frac{1}{2 \pi} \int_{0}^{\pi}(1+y-2 \sqrt{y} \cos \theta)^{\ell} d \theta
$$




$$
\begin{aligned}
& -\beta_{x} \frac{1}{2 \pi \mathbf{i}} \oint z^{\ell} \frac{y \underline{m}^{3}(z)(1+\underline{m}(z))^{-3}}{1-y \underline{m}^{2}(z)(1+\underline{m}(z))^{-2}} d z \\
= & \frac{[a(y)]^{\ell}+[b(y)]^{\ell}}{4}-\frac{1}{2} \sum_{\ell_{1}=0}^{\ell}\left(\begin{array}{c}
\ell \\
\ell_{1}
\end{array}\right)^{2} y^{\ell_{1}}+\beta_{x} \sum_{\ell_{2}=2}^{\ell}\left(\begin{array}{c}
\ell \\
\ell_{2}-2
\end{array}\right)\left(\begin{array}{l}
\ell \\
\ell_{2}
\end{array}\right) y^{\ell+1-\ell_{2}},
\end{aligned}
$$

where

$$
\begin{aligned}
& \frac{1}{2 \pi \mathbf{i}} \oint z^{\ell} \frac{y \underline{m}^{3}(z)(1+\underline{m}(z))^{-3}}{1-y \underline{m}^{2}(z)(1+\underline{m}(z))^{-2}} d z \\
= & \frac{y}{2 \pi \mathbf{i}} \oint\left(-\frac{1}{\underline{m}(z)}+\frac{y}{1+\underline{m}(z)}\right)^{\ell} \frac{\underline{m}(z)}{(1+\underline{m}(z))^{3}} d \underline{m}(z) \\
= & \left.\frac{y}{2 \pi \mathbf{i}} \oint \sum_{\ell_{1}=0}^{\ell} \frac{\underline{m}^{\ell_{1}}(z)(1+\underline{m}(z))^{\ell+3-\ell_{1}}}{\ell_{1}}\right)(-1)^{j} y^{\ell-\ell_{1}} \underline{m}(z) \\
= & \frac{y}{2 \pi \mathbf{i}} \oint \sum_{\ell_{1}=2}^{\ell} \frac{\left(\begin{array}{l}
\ell \\
\ell_{1}
\end{array}\right)(-1)^{j} y^{\ell-\ell_{1}}}{(z)(1+\underline{m}(z))^{\ell+3-\ell_{1}}} d \underline{m}(z) \\
= & \sum_{\ell_{1}=2}^{\ell} \frac{\left(\begin{array}{l}
\ell \\
\ell_{1}
\end{array}\right)(-1)^{j} y^{\ell+1-\ell_{1}}}{2 \pi \mathbf{i}} \oint \frac{1}{\underline{m}^{\ell_{1}-1}(z)(1+\underline{m}(z))^{\ell+3-\ell_{1}}} d \underline{m}(z) \\
= & -\sum_{\ell_{1}=2}^{\ell}\left(\begin{array}{c}
\ell \\
\ell_{1}-2
\end{array}\right)\left(\begin{array}{l}
\ell \\
\ell_{1}
\end{array}\right) y^{\ell+1-\ell_{1}} .
\end{aligned}
$$

and

$$
\begin{aligned}
& \quad \operatorname{Cov}\left(X_{f_{\ell}}, X_{f_{\ell^{\prime}}}\right) \\
& =2 y^{\ell+\ell^{\prime}} \sum_{\ell_{1}=0}^{\ell-1} \sum_{\ell_{2}=0}^{\ell^{\prime}}\left(\begin{array}{l}
\ell \\
\ell_{1}
\end{array}\right)\left(\begin{array}{l}
\ell^{\prime} \\
\ell_{2}
\end{array}\right)\left(\frac{1-y}{y}\right)^{\ell+\ell^{\prime}} \sum_{\ell_{3}=0}^{\ell-\ell_{1}} \ell_{3}\left(\begin{array}{c}
2 \ell-1-\ell_{1}-\ell_{3} \\
\ell-1
\end{array}\right)\left(\begin{array}{c}
2 \ell^{\prime}-1-\ell_{2}+\ell_{3} \\
\ell^{\prime}-1
\end{array}\right) \\
& +\beta_{x} y \sum_{\ell_{1}=1}^{\ell}\left(\begin{array}{c}
\ell \\
\ell_{1}-1
\end{array}\right)\left(\begin{array}{l}
\ell \\
\ell_{1}
\end{array}\right) y^{\ell-\ell_{1}} \cdot \sum_{\ell_{2}=1}^{\ell^{\prime}}\left(\begin{array}{c}
\ell^{\prime} \\
\ell_{2}-1
\end{array}\right)\left(\begin{array}{l}
\ell^{\prime} \\
\ell_{2}
\end{array}\right) y^{\ell^{\prime}-\ell_{2}}, \quad \ell, \ell^{\prime} \in\{1, \ldots, L\}
\end{aligned}
$$

with

$$
\begin{aligned}
\frac{1}{2 \pi \mathbf{i}} \oint \frac{z^{\ell}}{(1+\underline{m}(z))^{2}} d \underline{m}(z) & =\frac{1}{2 \pi \mathbf{i}} \oint \frac{1}{(1+\underline{m}(z))^{2}}\left(-\frac{1}{\underline{m}(z)}+\frac{y}{1+\underline{m}(z)}\right)^{\ell} d \underline{m}(z) \\
& =\frac{1}{2 \pi \mathbf{i}} \oint \sum_{\ell_{1}=0}^{\ell} \frac{\left(\begin{array}{l}
\ell \\
\ell_{1}
\end{array}\right)(-1)^{j} y^{\ell-\ell_{1}}}{\underline{m}_{1}(z)(1+\underline{m}(z))^{\ell+2-\ell_{1}}} d \underline{m}(z) \\
& =\sum_{\ell_{1}=1}^{\ell} \frac{\left(\begin{array}{l}
\ell \\
\ell_{1}
\end{array}\right)(-1)^{j} y^{\ell-\ell_{1}}}{2 \pi i} \oint \frac{1}{\underline{m}^{\ell_{1}}(z)[1+\underline{m}(z)]^{\ell+2-\ell_{1}}} d \underline{m}(z)
\end{aligned}
$$




$$
=\sum_{\ell_{1}=1}^{\ell}\left(\begin{array}{c}
\ell \\
\ell_{1}-1
\end{array}\right)\left(\begin{array}{l}
\ell \\
\ell_{1}
\end{array}\right) y^{\ell-\ell_{1}}
$$

\section{A Mathematical Tools}

Lemma A.1. [Burkholder (1973)]. Let $\left\{X_{k}\right\}$ be a complex martingale difference sequence with respect to the increasing $\sigma$-field $\left\{\mathscr{F}_{k}\right\}$. Then for $p>1$

$$
\mathrm{E}\left|\sum X_{k}\right|^{p} \leq K_{p} \mathrm{E}\left(\sum\left|X_{k}\right|^{2}\right)^{p / 2}
$$

Lemma A.2. For $\mathbf{X}=\left(X_{1}, \ldots, X_{n}\right)^{T}$ i.i.d. standardized (complex) entries, $\mathbf{C}=\left(c_{i j}\right)$ $n \times n$ matrix (complex) we have

$$
\mathrm{E}\left|\mathbf{X}^{*} \mathbf{C X}-\operatorname{tr} \mathbf{C}\right|^{4} \leq K\left(\left(\operatorname{tr}\left(\mathbf{C C}^{*}\right)\right)^{2}+\sum_{i=1}^{n} \mathrm{E}\left|X_{i i}^{8}\right|\left|c_{i i}\right|^{4}\right)
$$

The proof of the lemma can easily follow by simple calculus and thus omitted.

Lemma A.3. Let $f_{1}, f_{2}, \ldots$ be analytic in $D$, a connected open set of $\mathbb{C}$, satisfying $\left|f_{n}(z)\right| \leq$ $M$ for every $n$ and $z$ in $D$, and $f_{n}(z)$ converges, as $n \rightarrow \infty$ for each $z$ in a subset of $D$ having a limit point in $D$. Then there exists a function $f$, analytic in $D$ for which $f_{n}(z) \rightarrow f(z)$ and $f_{n}^{\prime}(z) \rightarrow f^{\prime}(z)$ for all $z \in D$ where' denotes the derivative. Moreover, on any set bounded by a contour interior to $D$ the convergence is uniform and $\left\{f_{n}^{\prime}(z)\right\}$ is uniformly bounded.

Lemma A.4. [Theorem 35.12 of Billingsley (1995)]. Suppose for each $n Y_{n 1}, Y_{n 2}, \ldots$, $Y_{n r_{n}}$ is a real martingale difference sequence with respect to the increasing $\sigma$-field $\left\{\mathscr{F}_{n j}\right\}$ having second moments. If as $n \rightarrow \infty$

$$
\sum_{j=1}^{r_{n}} \mathrm{E}\left(Y_{n j}^{2} \mid \mathscr{F}_{n, j-1}\right) \stackrel{i . p .}{\rightarrow} \sigma^{2}
$$

where $\sigma^{2}$ is a positive constant, and for each $\epsilon>0$

$$
\sum_{j=1}^{r_{n}} \mathrm{E}\left(Y_{n j}^{2} I_{\left(\left|Y_{n j}\right| \geq \epsilon\right)}\right) \rightarrow 0
$$

then

$$
\sum_{j=1}^{r_{n}} Y_{n r_{n}} \downarrow N\left(0, \sigma^{2}\right)
$$


Lemma A.5. (Lemma 2.6 of Bai (1999)). For $p \times n$ Hermitian A and B

$$
\left\|F^{\mathbf{A A} *}-F^{\mathbf{B B}^{*}}\right\| \leq p^{-1} \operatorname{rank}(\mathbf{A}-\mathbf{B}),
$$

where $\|\cdot\|$ here denotes sup norm on functions.

Lemma A.6. (Lemma 2.7 of Bai (1999)). For $p \times n$ Hermitian A and B

$$
L^{4}\left(F^{\mathbf{A A}^{*}}, F^{\mathbf{B B}^{*}}\right) \leq 2 p^{-2} \operatorname{tr}(\mathbf{A}-\mathbf{B})\left(\mathbf{A}^{*}-\mathbf{B}^{*}\right) \operatorname{tr}\left(\mathbf{A} \mathbf{A}^{*}+\mathbf{B B}^{*}\right),
$$

where $L(F, G)$ denotes the Levy distance between distribution functions.

Lemma A.7. (Lemma 2.6 of Silverstein and Bai (1995)). Let $z \in \mathbb{C}^{+}$with $v=\Im z$, A and $\mathbf{B}$ being $n \times n$ with $\mathbf{B}$ Hermitian, and $\mathbf{r} \in \mathbb{C}^{n}$. Then

$$
\left|\operatorname{tr}\left((\mathbf{B}-z \mathbf{I})^{-1}-\left(\mathbf{B}+\mathbf{r r}^{*}-z \mathbf{I}\right)^{-1}\right) \mathbf{A}\right|=\left|\frac{\mathbf{r}^{*}(\mathbf{B}-z \mathbf{I})^{-1} \mathbf{A}(\mathbf{B}-z \mathbf{I})^{-1} \mathbf{r}}{1+\mathbf{r}^{*}(\mathbf{B}-z \mathbf{I})^{-1} \mathbf{r}}\right| \leq \frac{\|\mathbf{A}\|}{v}
$$

\section{B Truncation and normalization for the proof of The- orem 2.1}

\section{B.1 Truncation of the matrix $\mathrm{Q}$}

Because $H$ is a proper distribution function, for any given $\epsilon>0$, there exists a constant $\tau>0$ such that $1-H(\tau)<\epsilon$. Without loss of generality, we may assume that $\tau$ is a continuity point of $H$. Suppose the singular value decomposition of $\mathbf{Q}$ is given by

$$
\mathrm{Q}=\mathbf{U} \Lambda \mathbf{V}^{*}
$$

where $\mathbf{U}_{p \times p}$ and $\mathbf{V}_{k \times k}$ are two unitary matrices and $\boldsymbol{\Lambda}_{p \times k}=\operatorname{diag}\left[l_{1}, l_{2}, \cdots\right]$ is a diagonal matrix of nonnegative real singular values of $\mathbf{Q}$ in descending order. Define

$$
\widehat{\mathbf{Q}}=\mathbf{U} \operatorname{diag}\left[l_{1} \wedge \sqrt{\tau}, \cdots, l_{k} \wedge \sqrt{\tau}, \cdots\right] \mathbf{V}^{*}
$$

and $\widehat{\mathbf{B}}_{n}=\frac{1}{n} \widehat{\mathbf{Q}} \mathbf{X}_{n} \mathbf{X}_{n}^{*} \widehat{\mathbf{Q}}^{*}$. By Lemma $\mathrm{A.5}$, we have

$$
\left\|F^{\mathbf{B}_{n}}-F^{\widehat{\mathbf{B}}_{n}}\right\| \leq \frac{1}{p} \operatorname{rank}(\mathbf{Q}-\widehat{\mathbf{Q}}) \leq \frac{1}{p} \#\left\{i: l_{i}^{2}>\tau\right\} \rightarrow 1-H(\tau)<\epsilon .
$$

Therefore, we may assume that the norm of $\mathbf{Q}$ is bounded by some constant $\sqrt{\tau}$. 


\section{B.2 Truncation}

By Assumption (a), there exists a sequence of constants $\eta_{n} \downarrow 0$ such that

$$
\frac{1}{p n \eta_{n}^{2}} \sum_{i=1}^{k} \sum_{j=1}^{n}\left\|\mathbf{q}_{i}\right\|^{2} \mathrm{E}\left|x_{i j}^{2}\right| I\left(\left|x_{i j}\right|>\eta_{n} \sqrt{n} /\left\|\mathbf{q}_{i}\right\|\right) \rightarrow 0 .
$$

Define $\widehat{x}_{i j}=x_{i j} I\left(\left|x_{i j}\right| \leq \eta_{n} \sqrt{n} /\left\|\mathbf{q}_{i}\right\|\right), \widehat{\mathbf{X}}_{n}=\left(\widehat{x}_{i j}\right)$ and $\widehat{\mathbf{B}}_{n}=n^{-1} \mathbf{Q} \widehat{\mathbf{X}}_{n} \widehat{\mathbf{X}}_{n}^{*} \mathbf{Q}_{n}^{*}$. Applying Lemma A.5 again, we have

$$
\left\|F^{\mathbf{B}_{n}}-F^{\widehat{\mathbf{B}}_{n}}\right\| \leq p^{-1} \operatorname{rank}\left(\mathbf{X}_{n}-\widehat{\mathbf{X}}_{n}\right) \leq p^{-1} \sum_{i=1}^{k} \sum_{j=1}^{n} I\left(\left|x_{i j}\right|>\eta_{n} \sqrt{n} /\left\|\mathbf{q}_{i}\right\|\right) \rightarrow 0 \text { a.s. }
$$

because

$$
\begin{aligned}
& \mathrm{E}\left(p^{-1} \sum_{i=1}^{k} \sum_{j=1}^{n} I\left(\left|x_{i j}\right|>\eta_{n} \sqrt{n} /\left\|\mathbf{q}_{i}\right\|\right)\right) \\
\leq & \left(p n \eta_{n}^{2}\right)^{-1} \sum_{i=1}^{k} \sum_{j=1}^{n}\left\|\mathbf{q}_{i}\right\|^{2} \mathrm{E}\left|x_{i j}^{2}\right| I\left(\left|x_{i j}\right|>\eta_{n} \sqrt{n} /\left\|\mathbf{q}_{i}\right\|\right) \rightarrow 0 \\
& \operatorname{Var}\left(p^{-1} \sum_{i=1}^{k} \sum_{j=1}^{n} I\left(\left|x_{i j}\right|>\eta_{n} \sqrt{n} /\left\|\mathbf{q}_{i}\right\|\right)\right) \\
\leq & p^{-2} \sum_{i=1}^{k} \sum_{j=1}^{n} \mathrm{P}\left(\left|x_{i j}\right|>\eta_{n} \sqrt{n} /\left\|\mathbf{q}_{i}\right\|\right) \\
\leq & \left(p^{2} n \eta_{n}^{2}\right)^{-1} \sum_{i=1}^{k} \sum_{j=1}^{n}\left\|\mathbf{q}_{i}\right\|^{2} \mathrm{E}\left|x_{i j}^{2}\right| I\left(\left|x_{i j}\right|>\eta_{n} \sqrt{n} /\left\|\mathbf{q}_{i}\right\|\right) \\
= & o\left(p^{-1}\right)
\end{aligned}
$$

and by Bernstein's inequality we have $P\left(p^{-1} \sum_{i=1}^{k} \sum_{j=1}^{n} I\left(\left|x_{i j}\right|>\eta_{n} \sqrt{n} /\left\|\mathbf{q}_{i}\right\|\right)>\epsilon\right) \leq$ $K \exp (-b p)$, for some constants $K<\infty$ and $b>0$.

\section{B.3 Centralization}

Define $\widetilde{\mathbf{X}}_{n}=\widehat{\mathbf{X}}_{n}-\mathrm{E} \widehat{\mathbf{X}}_{n}$ and $\widetilde{\mathbf{B}}_{n}=n^{-1} \mathbf{Q} \widetilde{\mathbf{X}}_{n} \widetilde{\mathbf{X}}_{n}^{*} \mathbf{Q}^{*}$. By Lemma A.6, we have

$$
L^{4}\left(F^{\widehat{\mathbf{B}}_{n}}, F^{\widetilde{\mathbf{B}}_{n}}\right) \leq 2 p^{-2} n^{-2} \operatorname{tr} \mathbf{Q}\left(\widehat{\mathbf{X}}_{n}-\widetilde{\mathbf{X}}_{n}\right)\left(\widehat{\mathbf{X}}_{n}-\widetilde{\mathbf{X}}_{n}\right)^{*} \mathbf{Q}^{*} \operatorname{tr}\left(\mathbf{Q} \widehat{\mathbf{X}}_{n} \widehat{\mathbf{X}}_{n}^{*} \mathbf{Q}^{*}+\mathbf{Q} \widetilde{\mathbf{X}}_{n} \widetilde{\mathbf{X}}_{n}^{*} \mathbf{Q}^{*}\right) .
$$


Notice that

$$
\begin{aligned}
& (p n)^{-1} \operatorname{tr}\left(\mathbf{Q}\left(\widehat{\mathbf{X}}_{n}-\widetilde{\mathbf{X}}_{n}\right)\left(\widehat{\mathbf{X}}_{n}-\widetilde{\mathbf{X}}_{n}\right)^{*} \mathbf{Q}^{*}\right) \\
= & (p n)^{-1} \operatorname{tr} \mathbf{Q E} \widehat{\mathbf{X}}_{n}\left(\mathrm{E} \widehat{\mathbf{X}}_{n}\right)^{*} \mathbf{Q}^{*} \\
= & (p n)^{-1} \sum_{i=1}^{p} \sum_{j=1}^{n}\left|\sum_{\ell=1}^{k} q_{i \ell} \mathrm{E} \widehat{x}_{\ell j}\right|^{2} \\
\leq & n^{-1} \sum_{i=1}^{p} \sum_{j=1}^{n} \sum_{\ell=1}^{k}\left|q_{i \ell}\right|^{2} \sum_{h=1}^{k} \frac{\left\|\mathbf{q}_{h}\right\|^{2}}{\eta_{n}^{2} p n} \mathrm{E}\left|x_{h j}^{2}\right| I\left(\left|x_{h j}\right|>\eta_{n} \sqrt{n} /\left\|\mathbf{q}_{h}\right\|\right)
\end{aligned}
$$

(by Cauchy-Schwarz inequality)

$$
\begin{aligned}
& =n^{-1} \sum_{i=1}^{p} \sum_{\ell=1}^{k}\left|q_{i \ell}\right|^{2} \cdot\left(\eta_{n}^{2} p n\right)^{-1} \sum_{j=1}^{n} \sum_{h=1}^{k}\left\|\mathbf{q}_{h}\right\|^{2} \mathrm{E}\left|x_{h j}^{2}\right| I\left(\left|x_{h j}\right|>\eta_{n} \sqrt{n} /\left\|\mathbf{q}_{h}\right\|\right) \\
& =n^{-1} \operatorname{tr}\left(\mathbf{Q Q}^{*}\right)\left(\eta_{n}^{2} p n\right)^{-1} \sum_{j=1}^{n} \sum_{h=1}^{k}\left\|\mathbf{q}_{h}\right\|^{2} \mathrm{E}\left|x_{h j}^{2}\right| I\left(\left|x_{h j}\right|>\eta_{n} \sqrt{n} /\left\|\mathbf{q}_{h}\right\|\right) \\
& =o(1) .
\end{aligned}
$$

Noticing that the above bound is non-random, to show that $L^{4}\left(F^{\widehat{\mathbf{B}}_{n}}, F^{\widetilde{\mathbf{B}}_{n}}\right) \rightarrow 0$, a.s., one only needs to prove that

$$
\begin{aligned}
& \frac{1}{p n} \operatorname{tr}\left(\mathbf{Q} \widetilde{\mathbf{X}}_{n} \widetilde{\mathbf{X}}_{n}^{*} \mathbf{Q}^{*}\right)=\frac{1}{p n} \sum_{i=1}^{p} \sum_{j=1}^{n}\left|\sum_{\ell=1}^{k} q_{i \ell} \widetilde{x}_{\ell j}\right|^{2} \\
= & \frac{1}{p n} \sum_{i=1}^{p} \sum_{j=1}^{n} \sum_{\ell=1}^{k}\left|q_{i \ell}^{2} \| \widetilde{x}_{\ell j}^{2}\right|+\frac{1}{p n} \sum_{i=1}^{p} \sum_{j=1}^{n} \sum_{k_{1} \neq k_{2}} q_{i k_{1}} \bar{q}_{i k_{2}} \widetilde{x}_{k_{1}, j} \overline{\widetilde{x}}_{k_{2}, j}=O_{\text {a.s. }}(1) .
\end{aligned}
$$

Note that

$$
\mathrm{E}\left(\frac{1}{p n} \sum_{i=1}^{p} \sum_{j=1}^{n} \sum_{\ell=1}^{k}\left|q_{i k}^{2}\right|\left|\widetilde{x}_{\ell j}^{2}\right|\right) \leq \frac{1}{p n} \sum_{i=1}^{p} \sum_{j=1}^{n} \sum_{\ell=1}^{k}\left|q_{i \ell}^{2}\right|=O(1)
$$

and

$$
\begin{aligned}
& \mathrm{E}\left(\frac{1}{p n} \sum_{i=1}^{p} \sum_{j=1}^{n} \sum_{\ell=1}^{k}\left|q_{i \ell}^{2}\right|\left(\left|\widetilde{x}_{\ell j}^{2}\right|-\mathrm{E}\left|\widetilde{x}_{\ell j}^{2}\right|\right)\right)^{4} \\
\leq & \frac{1}{p^{4} n^{4}} \sum_{j=1}^{n} \sum_{\ell=1}^{k}\left(\sum_{i=1}^{p}\left|q_{i \ell}^{2}\right|\right)^{4} \mathrm{E}\left|\widetilde{x}_{\ell j}^{8}\right|+\frac{3}{p^{4} n^{4}}\left(\sum_{j=1}^{n} \sum_{\ell=1}^{k}\left(\sum_{i=1}^{p}\left|q_{i \ell}^{2}\right|\right)^{2} \mathrm{E}\left|\widetilde{x}_{\ell j}^{4}\right|\right)^{2} \\
\leq & \frac{\eta_{n}^{6}}{p^{4}} \sum_{\ell=1}^{k}\left\|\mathbf{q}_{\ell}\right\|^{2}+\frac{3 \eta_{n}^{4}}{p^{4}}\left(\sum_{\ell=1}^{k}\left\|\mathbf{q}_{\ell}\right\|^{2}\right)^{2}=o\left(p^{-2}\right) .
\end{aligned}
$$


These inequalities simply imply $(p n)^{-1} \sum_{i=1}^{p} \sum_{j=1}^{n} \sum_{\ell=1}^{k}\left|q_{i \ell}^{2}\right|\left|\widetilde{x}_{\ell j}^{2}\right|=O_{a . s .}(1)$. Furthermore,

$$
\begin{aligned}
& \mathrm{E}\left(\frac{1}{p n} \sum_{i=1}^{p} \sum_{j=1}^{n} \sum_{k_{1} \neq k_{2}} q_{i k_{1}} \bar{q}_{i k_{2}} \widetilde{x}_{k_{1}, j} \overline{\widetilde{x}}_{k_{2}, j}\right)^{2} \leq \frac{2}{p^{2} n^{2}} \sum_{j=1}^{n} \sum_{k_{1} \neq k_{2}}\left|\sum_{i=1}^{p} q_{i k_{1}} \bar{q}_{i k_{2}}\right|^{2} \\
\leq & \frac{2}{p^{2} n} \operatorname{tr}\left(\mathbf{Q Q}^{*}\right)^{2}=O\left(p^{-2}\right),
\end{aligned}
$$

which implies that $(p n)^{-1} \sum_{i=1}^{p} \sum_{j=1}^{n} \sum_{k_{1} \neq k_{2}} q_{i k_{1}} \bar{q}_{i k_{2}} \widetilde{x}_{k_{1}, j} \overline{\widetilde{x}}_{k_{2}, j} \rightarrow 0$, a.s. Hence the assertion (B.3) is proved.

\section{B.4 Rescaling}

Denote $\sigma_{i j}^{2}=\mathrm{E}\left|\widetilde{x}_{i j}\right|^{2}$, and $y_{i j}$ are i.i.d. random variables satisfy $\mathrm{P}\left(y_{i j}= \pm 1\right)=\frac{1}{2}$ and are independent of all $x_{i j}$ 's. Define

$$
\breve{x}_{i j}= \begin{cases}\sigma_{i j}^{-1} \widetilde{x}_{i j} & \text { if } \sigma_{i j}^{2}>1-\eta_{n} \\ y_{i j} & \text { otherwise. }\end{cases}
$$

We further define $\breve{\mathbf{X}}_{n}=\left(\breve{x}_{i j}\right)$ and $\breve{\mathbf{B}}_{n}=n^{-1} \mathbf{Q} \breve{\mathbf{X}}_{n} \breve{\mathbf{X}}_{n}^{*} \mathbf{Q}^{*}$. Applying Lemma A.6 again, we have

$$
L^{4}\left(F^{\breve{\mathbf{B}}_{n}}, F^{\widetilde{\mathbf{B}}_{n}}\right) \leq 2 p^{-2} n^{-1} \operatorname{tr} \mathbf{Q}\left(\widetilde{\mathbf{X}}_{n}-\breve{\mathbf{X}}_{n}\right)\left(\widetilde{\mathbf{X}}_{n}-\breve{\mathbf{X}}_{n}\right)^{*} \mathbf{Q}^{*} \cdot \operatorname{tr}\left(\widetilde{\mathbf{B}}_{n}+\breve{\mathbf{B}}_{n}\right)
$$

We have proved in (B.3) that $p^{-1} \operatorname{tr} \widetilde{\mathbf{B}}_{n}=O_{\text {a.s. }}$ (1). Similarly, we can prove that $p^{-1} \operatorname{tr} \breve{\mathbf{B}}_{n}=$ $O_{\text {a.s. }}(1)$. What remains is to show that

$$
\frac{1}{p n} \operatorname{tr} \mathbf{Q}\left(\widetilde{\mathbf{X}}_{n}-\breve{\mathbf{X}}_{n}\right)\left(\widetilde{\mathbf{X}}_{n}-\breve{\mathbf{X}}_{n}\right)^{*} \mathbf{Q}^{*}=\frac{1}{p n} \sum_{i=1}^{p} \sum_{j=1}^{n}\left|\sum_{\ell=1}^{k} q_{i \ell}\left(\breve{x}_{\ell j}-\widetilde{x}_{\ell j}\right)\right|^{2}=o_{a . s .}(1) .
$$

Write $E_{n}=\left\{(i, j): \sigma_{i j}^{2}<1-\eta_{n}\right\}$ and $E_{(j)}=\left\{i:(i, j) \in E_{n}\right\}$. Then

$$
\begin{aligned}
\sum_{i=1}^{p} \sum_{j=1}^{n}\left|\sum_{\ell=1}^{k} q_{i \ell}\left(\breve{x}_{\ell j}-\widetilde{x}_{\ell j}\right)\right|^{2}= & \sum_{i=1}^{p} \sum_{j=1}^{n} \sum_{\ell \in E_{(j)}}\left|q_{i \ell}^{2}\right|\left|\breve{x}_{\ell j}-\widetilde{x}_{\ell j}\right|^{2} \\
& +\sum_{i=1}^{p} \sum_{j=1}^{n} \sum_{k_{1} \neq k_{2}} q_{i k_{1}} \bar{q}_{i k_{2}}\left(\breve{x}_{k_{1} j}-\widetilde{x}_{k_{1 j} j}\right)\left(\overline{\breve{x}}_{k_{2} j}-\overline{\widetilde{x}}_{k_{2} j}\right) .
\end{aligned}
$$

Note that $\sum_{i=1}^{p}\left|q_{i \ell}\right|^{2} \leq\left\|\mathbf{q}_{\ell}\right\|^{2}$ and hence

$$
\mathrm{E}\left(\frac{1}{p n} \sum_{i=1}^{p} \sum_{j=1}^{n} \sum_{\ell \in E_{(j)}}\left|q_{i \ell}^{2}\right|\left|\breve{x}_{\ell j}-\widetilde{x}_{\ell j}\right|^{2}\right)
$$




$$
\begin{aligned}
& \leq(p n)^{-1}\left(\sum_{(i, j) \in E_{n}}\left\|\mathbf{q}_{i}\right\|^{2} \mathrm{E}\left|y_{i j}-\widetilde{x}_{i j}\right|^{2}+\sum_{(i, j) \notin E_{n}}\left\|\mathbf{q}_{i}^{2}\right\|\left(1-\sigma_{i j}^{-1}\right)^{2}\right) \\
& \leq(p n)^{-1}\left(\sum_{(i, j) \in E_{n}}\left\|\mathbf{q}_{i}\right\|^{2}\left(1+\sigma_{i j}^{2}\right)+\eta_{n}^{2} n \sum_{i=1}^{k}\left\|\mathbf{q}_{i}^{2}\right\|\right) \\
& \leq 2\left(p n \eta_{n}^{2}\right)^{-1} \sum_{i, j}\left\|\mathbf{q}_{i}\right\|^{2}\left(1-\sigma_{i j}^{2}\right)+\frac{\eta_{n}}{p} \operatorname{tr}\left(\mathbf{Q Q}^{*}\right) \\
& \leq 2\left(p n \eta_{n}^{2}\right)^{-2} \sum_{i, j}\left\|\mathbf{q}_{i}\right\|^{2} \mathrm{E}\left|x_{i j}^{2}\right| I\left(\left|x_{i j}\right|>\eta_{n} \sqrt{n} /\left\|\mathbf{q}_{i}\right\|\right)+\eta_{n}\|\mathbf{Q}\|^{2} \rightarrow 0 .
\end{aligned}
$$

Similar to (B.4) and (B.5), one can prove that

$$
\begin{gathered}
\mathrm{E}\left(\frac{1}{p n} \sum_{i=1}^{p} \sum_{j=1}^{n} \sum_{\ell \in E_{(j)}}\left|q_{i \ell}^{2}\right|\left(\left|\breve{x}_{\ell j}-\widetilde{x}_{\ell j}\right|^{2}-\mathrm{E}\left|\breve{x}_{\ell j}-\widetilde{x}_{\ell j}\right|^{2}\right)\right)^{4}=O\left(p^{-2} \eta_{n}^{-4}\right) \\
\operatorname{Var}\left(\frac{1}{p n} \sum_{i=1}^{p} \sum_{j=1}^{n} \sum_{k_{1} \neq k_{2}} q_{i k_{1}} \bar{q}_{i_{k}}\left(\breve{x}_{k_{1 j} j}-\widetilde{x}_{k_{1} j}\right)\left(\overline{\widetilde{x}}_{k_{2 j} j}-\overline{\widetilde{x}}_{k_{2 j} j}\right)\right)=O\left(p^{-2}\right) .
\end{gathered}
$$

From these, it is easy to show (B.6).

\section{Truncation and normalization for the proof of The- orem 3.1}

\section{C.1 Truncation}

By Assumption (c), there exists a sequence of constants $\eta_{n} \downarrow 0$ such that

$$
\frac{1}{p n \eta_{n}^{6}} \sum_{i=1}^{k} \sum_{j=1}^{n}\left\|\mathbf{q}_{i}\right\|^{2} \mathrm{E}\left|x_{i j}^{4}\right| I\left(\left|x_{i j}\right|>\eta_{n} \sqrt{n /\left\|\mathbf{q}_{i}\right\|}\right) \rightarrow 0,
$$

Define $\widehat{x}_{i j}=x_{i j} I\left(\left|x_{i j}\right| \leq \eta_{n} \sqrt{n /\left\|\mathbf{q}_{i}\right\|}\right), \widehat{\mathbf{X}}_{n}=\left(\widehat{x}_{i j}\right)$ and $\widehat{\mathbf{B}}_{n}=n^{-1} \mathbf{Q} \widehat{\mathbf{X}}_{n} \widehat{\mathbf{X}}_{n}^{*} \mathbf{Q}_{n}^{*}$. Then,

$$
\begin{aligned}
\mathrm{P}\left(\mathbf{B}_{n} \neq \widehat{\mathbf{B}}_{n}\right) & \leq \mathrm{E} \sum_{i=1}^{k} \sum_{j=1}^{n} I\left(\left|x_{i j}\right|>\eta_{n} \sqrt{n /\left\|\mathbf{q}_{i}\right\|}\right) \\
& \leq \eta_{n}^{-4} n^{-2} \sum_{i=1}^{k} \sum_{j=1}^{n}\left\|\mathbf{q}_{i}\right\|^{2} \mathrm{E}\left|x_{i j}^{4}\right| I\left(\left|x_{i j}\right|>\eta_{n} \sqrt{n /\left\|\mathbf{q}_{i}\right\|}\right) \rightarrow 0 .
\end{aligned}
$$




\section{C.2 Centralization}

Similarly define $\widetilde{\mathbf{X}}_{n}=\widehat{\mathbf{X}}_{n}-\mathbf{E} \widehat{\mathbf{X}}_{n}$ and $\widetilde{\mathbf{B}}_{n}=n^{-1} \mathbf{Q} \widetilde{\mathbf{X}}_{n} \widetilde{\mathbf{X}}_{n}^{*} \mathbf{Q}^{*}$. By Lemma A.6, we have

$$
L^{4}\left(F^{\widehat{\mathbf{B}}_{n}}, F^{\widetilde{\mathbf{B}}_{n}}\right) \leq n^{-2} p^{-2} \operatorname{tr}\left(\mathbf{Q}\left(\widehat{\mathbf{X}}_{n}-\widetilde{\mathbf{X}}_{n}\right)\left(\widehat{\mathbf{X}}_{n}-\widetilde{\mathbf{X}}_{n}\right)^{*} \mathbf{Q}^{*}\right) \operatorname{tr}\left(\mathbf{Q} \widehat{\mathbf{X}}_{n} \widehat{\mathbf{X}}_{n}^{*} \mathbf{Q}^{*}+\mathbf{Q} \widetilde{\mathbf{X}}_{n} \widetilde{\mathbf{X}}_{n}^{*} \mathbf{Q}^{*}\right) .
$$

Notice that

$$
\begin{aligned}
& (p n)^{-1} \operatorname{tr}\left(\mathbf{Q}\left(\widehat{\mathbf{X}}_{n}-\widetilde{\mathbf{X}}_{n}\right)\left(\widehat{\mathbf{X}}_{n}-\widetilde{\mathbf{X}}_{n}\right)^{*} \mathbf{Q}^{*}\right)=(p n)^{-1} \operatorname{tr} \mathbf{Q E} \widehat{\mathbf{X}}_{n}\left(\mathrm{E} \widehat{\mathbf{X}}_{n}\right)^{*} \mathbf{Q}^{*} \\
= & (p n)^{-1} \sum_{i=1}^{p} \sum_{j=1}^{n}\left|\sum_{\ell=1}^{k} q_{i \ell} \mathrm{E} \widehat{x}_{\ell j}\right|^{2} \quad \text { (by Cauchy-Schwarz inequality) } \\
\leq & (p n)^{-1} \sum_{i=1}^{p} \sum_{j=1}^{n} \sum_{\ell=1}^{k}\left|q_{i \ell}^{2}\right| \sum_{h=1}^{k} \frac{\left\|\mathbf{q}_{h}\right\|^{3}}{\eta_{n}^{h} n^{3}} \mathrm{E}\left|x_{\ell j}^{4}\right| I\left(\left|x_{h j}\right|>\eta_{n} \sqrt{n /\left\|\mathbf{q}_{\ell}\right\|}\right) \\
= & n^{-1} \sum_{i=1}^{p} \sum_{\ell=1}^{k}\left|q_{i \ell}^{2}\right| \cdot \sum_{j=1}^{n} \sum_{h=1}^{k} \frac{\left\|\mathbf{q}_{h}\right\|^{3}}{\eta_{n}^{6} n^{3}} \mathrm{E}\left|x_{\ell j}^{4}\right| I\left(\left|x_{h j}\right|>\eta_{n} \sqrt{n /\left\|\mathbf{q}_{\ell}\right\|}\right) \\
= & o(1) .
\end{aligned}
$$

By the similar approach given in subsection B.3, one may prove that

$$
(n p)^{-1}\left[\operatorname{tr}\left(\mathbf{Q} \widehat{\mathbf{X}}_{n} \widehat{\mathbf{X}}_{n}^{*} \mathbf{Q}^{*}\right)+\operatorname{tr}\left(\mathbf{Q} \widetilde{\mathbf{X}}_{n} \widetilde{\mathbf{X}}_{n}^{*} \mathbf{Q}^{*}\right)\right]=O_{a . s .}(1) .
$$

Hence $L^{4}\left(F^{\widehat{\mathbf{B}}_{n}}, F^{\widetilde{\mathbf{B}}_{n}}\right) \rightarrow 0$ a.s.

\section{C.3 Rescaling}

Denote $\sigma_{i j}^{2}=\mathrm{E}\left|\widetilde{x}_{i j}\right|^{2}$, and $y_{i j}$ are i.i.d. random variables satisfy $\mathrm{P}\left(y_{i j}= \pm 1\right)=\frac{1}{2}$. Define

$$
\breve{x}_{i j}= \begin{cases}\sigma_{i j}^{-1} \widetilde{x}_{i j} & \text { if } \sigma_{i j}^{2}>1-\eta_{n}, \\ y_{i j} & \text { otherwise. }\end{cases}
$$

We further define $\breve{\mathbf{X}}_{n}=\left(\breve{x}_{i j}\right)$ and $\breve{\mathbf{B}}_{n}=\frac{1}{n} \mathbf{Q} \breve{\mathbf{X}}_{n} \breve{\mathbf{X}}_{n}^{*} \mathbf{Q}^{*}$. Similar to subsection B.4, one can show that

$$
L^{4}\left(F^{\breve{\mathbf{B}}_{n}}, F^{\widetilde{\mathbf{B}}_{n}}\right) \rightarrow 0, \text { a.s. }
$$

\section{References}

Anderson, T. W. (2003). An Introduction to Multivariate Statistical Analysis. 3rd Edition. John Wiley \& Sons. 
Ahn, M., Shen, H., Lin, W., and Zhu, H., (2015) A sparse reduced rank framework for group analysis of functional neuroimaging data. Statistica Sinica, 25, 295-312.

Bai, Z. D., (1999) Methodologies in spectral analysis of large dimensional random matrices, a review. Statistica Sinica, 9, 611-677.

Bai, Z. D., Jiang, D. D., Yao, J. F. and Zheng, S. R. (2009). Corrections to LRT on large dimensional covariance matrix by RMT. Annals of Statistics, 37(6B), 3822-3840.

Bai, Z. D., Jiang, D. D., Yao, Jianfeng and Zheng, Shurong (2013). Testing linear hypotheses in high-dimensional regressions. Statistics, 47(6), 1207-1223.

Bai, Z. D. and Silverstein, J. W. (1998). No eigenvalues outside the support of the limiting spectral distribution of large-dimensional sample covariance matrices. Ann. Probab., 26(1), 316-345.

Bai, Z. D. and Silverstein, J. W. (2004). CLT for linear spectral statistics of large dimensional sample covariance matrices. Ann. Probab., 32(1), 553-605.

Bai, Z. D. and Silverstein, J. W. (2010). Spectral Analysis of Large Dimensional Random Matrices. Science Press: Beijing.

Bai, Z. D. and Zhou, W. (2008). Large sample covariance matrices without independence structures in columns. Statistica Sinica, 28, 425-442.

Banna, M. and Merlevède, F. (2015). Limiting spectral distribution of large sample covariance matrices associated with a class of stationary processes. J. Theor. Probab., 28, 745-783.

Billingsley, P. (1995). Probability and Measure. John Wiley \& Sons: New York

Burkholder, D. L. (1973). Distribution function inequalities for martingales. Ann. Probab., 1, 19-42.

Chen S. X., Zhang, L. X. and Zhong, P. S. (2010). Tests for high dimensional covariance matrices. Journal of the American Statistical Association, 105(490), 810-819.

Couillet, R. and Debbah, M. (2013). Signal processing in large systems: A new paradigm. IEEE Signal Processing Magazine, 30(1), 24-39.

Jiang, D. D., Bai, Z. D. and Zheng, S. R. (2013). Testing the independence of sets of large dimensional variabels. Scicence China Mathematics, 56(1), 135-147. 
Dobriban, E., (2015). Efficient computation of limit spectra of sample covariance matrices. Random Matrices: Theory and Applications, 4 (4), 1550019.

Jin, B. S., Wang, C., Bai, Z. D., Nair, K. K. and Harding, M. (2014). Limiting spectral distribution of a symmetrized auto-cross covariance matrix. Annals of Applied Probability, 24(3), 1199-1225.

Jin, B. S., Wang, C. and Miao, B. Q. (2009) Limiting spectral distribution of largedimensional sample covariance matrices generated by $V$ ARMA. Journal Of Multivariate Analysis, 100 No. 9 pp 2112-2125.

Johnstone, I. M. (2007). High dimensional statistical inference and random matrices. Int. Cong. Mathematicians, Vol. I, 307-333. Zürich, Switzerland: European Mathematical Society.

Jonsson, D. (1982). Some limit theorems for the eigenvalues of a sample covariance matrix. J. Multivariate Anal., 12, 1-38.

Lindquist, M. A. (2008). The statistical analysis of fMRI data. Statistical science, 23, 439-464.

Liu, B. S., Xu, L., Zheng, S. R. and Tian, G. L. (2014). A new test for the proportionality of two large dimensional covariance matrices. J. Multivariate Anal., 131, 293-308.

Marčenko, V. A. and Pastur, L. A. (1967). Distribution of eigenvalues for some sets of random matrices. Math. USSR-Sb, 1, 457-483.

Najim, J. and Yao, J. F.(2013). Gaussian fluctions for linear spectral staitstics of large random covairance matrices. arXiv: 1309.3728.

Pan, G. M. and Zhou, W. (2008). Central limit theorem for signal-to-interference ratio of reduced rank linear receiver. Ann. Appl. Probab., 18, 1232-1270.

Pan, G. M. (2012). Comparison between two types of large sample covariance matrices. Annales de l'Institut Henri Poincare-Probabiliteset Statistiques, 50(2), 655-677.

Paul, D. and Aue, A. (2014). Random matrix theory in statistics: A review. J. Statist. Planning and Inference, 150, 1-29.

Purdon, P. L., Solo, V., Weissko, R. M. and Brown, E. (2001). Locally regularized spatiotemporal modeling and model comparison for functional MRI. NeuroImage, 14, 912923. 
Silverstein, J. W. (1995). Strong convergence of the empirical distribution of eigenvalues of large dimensional random matrices. J. Multivariate Anal., 5 331-339.

Silverstein, J. W. and Bai, Z. D. (1995). On the empirical distribution of eigenvalues of a class of large dimensional random matrices. J. Multivariate Anal., 54, 175-192.

Silverstein, J. W. and Choi, S. I. (1995). Analysis of the limiting spectral distribution of large dimensional random matrices. J. Multivariate Anal., 54, 295-309.

Srivastava, M.S. (2005). Some tests concerning the covariance matrix in high dimensional data. J. Japan Statist. Soc., 35, 251-272.

Wang, C., Jin, B. S. and Miao, B. (2011) On limiting spectral distribution of large sample covariance matrices by $V A R M A(p, q)$. Journal of Time Series Analysis, 32(5), 539-546.

Wang, Q. and Yao, J. (2013). On the sphericity test with large-dimensional observations. Electronic J. Statist., 7, 2164-2192

Wachter K. W. (1980). The strong limits of random matrix spectra for sample matrices of independent elements. Ann. Probab., 6(1), 1-18.

Wong, W. K. and Miller, R. B. (1990). Repeated time series analysis of ARIMA-noise models. Journal of Business and Economic Statistics, 8(2) 243-250.

Wong, W. K., Miller, R. B. and Shrestha, K. (2001). Maximum likelihood estimation of ARMA models with error processes for replicated observations. Journal of Applied Statistical Science, 10, 287-297.

Yao, J. (2012) A note on a Marcenko-Pastur type theorem for time series. Statistics \&6 Probability Letters, 82(1), pp 22-28.

Zheng, S. R., Bai, Z. D. and Yao, J. F. (2015). Substitution principle for CLT of linear spectral statistics of high-dimensional sample covariance matrices with applications to hypothesis testing. Ann. Statist., 43(2), 546-591.

Zheng, S. R., Bai, Z. D. and Yao, J. F. (2015). CLT for large dimensional general Fisher matrices and its applications in high-dimensional data analysis. Forthcoming in Bernoulli. DOI: 10.13140/RG.2.1.3973.7765 